\title{
Nitrogen-Substitution in the Flapping Wings of Cyclooctatetraene-Fused Molecules
}

\author{
Kensuke Suga, ${ }^{1}$ Takuya Yamakado, ${ }^{1}$ and Shohei Saito*1 \\ ${ }^{1}$ Graduate School of Science, Kyoto University, Kitashirakawa Oiwake, Sakyo, Kyoto 606-8502, Japan
}

E-mail: s_saito@kuchem.kyoto-u.ac.jp

Shohei Saito

Shohei Saito received his Ph.D. degree from Kyoto University in 2010.

After working as an Assistant Professor of Nagoya University, he has been an Associate Professor of Kyoto University since 2016. His research interest is focused on flapping molecules (FLAP) for photofunctional systems. He received The Asian and Oceanian Photochemistry Association (APA) award for young scientist 2018.

\begin{abstract}
New synthetic protocols to the nitrogen-embedded flapping molecules have been developed. Gram-scale synthesis of a key precursor, tetraamine of dibenzo $[a, e]$ cyclooctatetraene has been established for designing flapping quinoxaline and flapping phenazineimide. The impact of the nitrogen substitution on the photophysical properties and the viscosity-probing function has been investigated in comparison with the reported flapping anthraceneimide.
\end{abstract}

Keywords: Excited-state planarization, Fluorescent molecule, Viscosity probe

\section{Introduction}

Flapping molecules (FLAP) with the formal $8 \pi$ ring at its center have attracted attention as a versatile photofunctional system. ${ }^{1}$ While dibenzo $[b, f]$ oxepin $^{2}$ has been recognized as a classical fluorophore with the large Stokes shift that shows a bent-toplanar conformational change in the lowest singlet excited state $\left(\mathrm{S}_{1}\right)$, a variety of functions and applications of the FLAP series have been recently demonstrated, such as multi-color fluorescent dye ${ }^{3}$ highly sensitive viscosity probe, ${ }^{4}$ ratiometric force probe (flexible mechanophore), ${ }^{5}$ and light-melt adhesive. ${ }^{6}$ The mechanism of the excited-state planarization has also been focused in relation to the excited-state aromaticity. ${ }^{7}$ In this context, structural diversity of the conformationally flexible molecules has been rapidly enriched in recent studies, such as $\pi$ expanded cyclooctatetraene (COT), ${ }^{3-6,8}$ dihydrophenazine, ${ }^{9}$ phenothiazine, ${ }^{10}$ dibenzoarsepin, ${ }^{11}$ as well as $\pi$-expanded oxepin. ${ }^{12}$ On the other hand, the effect of nitrogen substitution to the "wings" of the flapping molecules has not been studied, despite the efficacy of the nitrogen-doping has been widely recognized in tuning electronic structures and optical properties of polycyclic aromatic hydrocarbons (PAHs). ${ }^{13}$ Here, we have developed new synthetic protocols to the nitrogen-embedded flapping molecules bearing quinoxaline and phenazineimide wings (Figure 1). Since the flapping phenazineimide shows an environment-sensitive fluorescence, its photophysical properties and viscosity-probing function have been investigated in comparison with the previously reported flapping anthraceneimide.

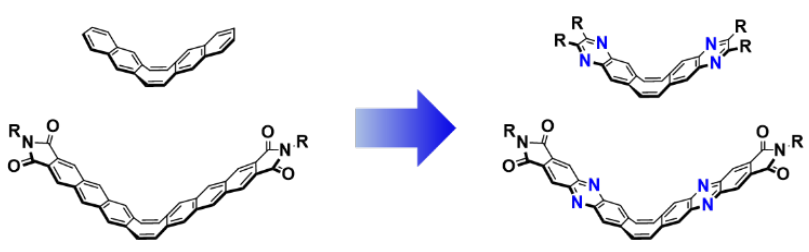

Figure 1. Nitrogen-substitution of the reported flapping naphthalene ${ }^{14}$ and flapping anthraceneimide ${ }^{3-5}$ (left), providing novel flapping quinoxaline and flapping phenazineimide (right).

\section{Results and Discussion}

To develop the synthetic route to the nitrogen-embedded flapping systems, a key precursor of dibenzo[ $[a, e]$ cyclooctatetraene(COT)-2,3,8,9-tetraamine was prepared from the previously reported tetrabromo-substituted dibenzo[a,e]COT 1 (Figure 2) ${ }^{4 \mathrm{~b}}$ First, Pd-catalyzed imination of 1 with benzophenone imine provided 2 in $81 \%$ yield. The following hydrolysis of $\mathbf{2}$ in acidic conditions led to an immediate precipitation of the corresponding tetraamine $\mathbf{3}$ in a protonated form. Facile filtration process realized gram-scale synthesis of $\mathbf{3}$. Since the non-protonated, neutralized form of $\mathbf{3}$ is highly electron-rich and thus gradually decomposed by spontaneous air oxidation, the protonated form of $\mathbf{3}$ was treated for the next reactions. Flapping quinoxaline FLAP1 was prepared by condensation of $\mathbf{3}$ with 3,4-hexanedione in $68 \%$ yield. Next, we attempted to synthesize flapping phenazine $\mathbf{5}$ (Figure S2.1), but the coupling reaction ${ }^{15}$ of $\mathbf{3}$ with 1,2-dibromo4,5-dimethylbenzene as well as the reaction of 1 with 4,5dimethylbenzene-1,2-diamine failed to give $\mathbf{5}$, probably due to the poor solubility of products. To improve the solubility, we designed flapping phenazineimide FLAP2 bearing bulky 2,6diisopropylphenyl substituents at the terminal positions. Pdcatalyzed coupling reaction of $\mathbf{3}$ with dibromo-substituted phthalimide successfully provided FLAP2 in 28\% yield. 
(a)

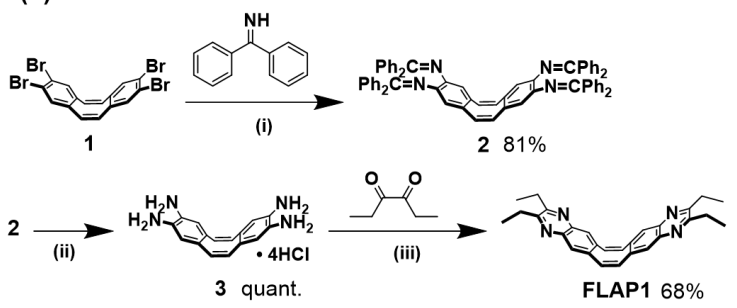

(b)

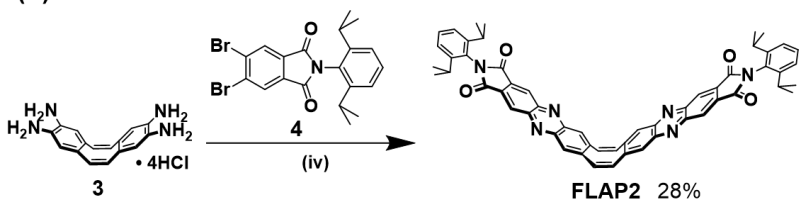

Figure 2. Synthesis of (a) FLAP1 and (b) FLAP2. Reagents and conditions: (i) $\mathrm{Ph}_{2} \mathrm{C}=\mathrm{NH}, \mathrm{Pd}_{2}(\mathrm{dba})_{3},( \pm)$-BINAP, $\mathrm{NaO}^{t} \mathrm{Bu}$, toluene, reflux, $42 \mathrm{~h}$; (ii) $\mathrm{HCl}, \mathrm{H}_{2} \mathrm{O} / \mathrm{THF}, 25^{\circ} \mathrm{C}$, $4.5 \mathrm{~h}$; (iii) 3,4hexanedione, $\mathrm{CHCl}_{3} / \mathrm{AcOH}, 70{ }^{\circ} \mathrm{C}, 18 \mathrm{~h}$; (iv) 4, SPhos Pd G2, SPhos, $\mathrm{Cs}_{2} \mathrm{CO}_{3}$, toluene, reflux, $18 \mathrm{~h}$.

Crystal structures of FLAP1 and FLAP2 were obtained with bent conformations (Figure 3). The COT bending angle, defined in Figure 5a, were $43.3^{\circ}$ and $42.2^{\circ}$ for FLAP1, and $40.3^{\circ}$ for FLAP2. Bond lengths of the central COT moieties indicated that two $\pi$ systems of the wings are not effectively conjugated due to the bent form of the COT ring, which is supported by the DFT calculations in the ground $\mathrm{S}_{0}$ state (Figure S6.9, S6.11). Consistent with the needle-like crystal morphology, the Vshaped FLAP1 molecules form a unique two-fold columnar $\pi$ stacking structure. The quinoxaline wings are tightly stacked on both sides, in which the interplanar distances are both $3.4 \AA$. In the block crystal of FLAP2 recrystallized from a chlorobenzene $/ \mathrm{PrOH}$ solution, the $\mathrm{V}$-shaped molecules are not $\pi$-stacked in the columnar manner due to the terminal bulky groups. Instead, the phenazineimide moiety forms a cofacial packing only on one side with the 3.4- $\AA$ interplanar distance. The other side of the phenazineimide unit sandwiches a chlorobenzene molecule with two FLAP2 molecules.

(a)

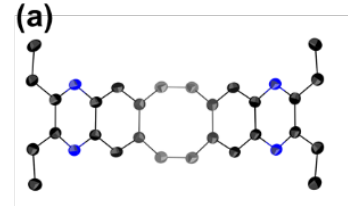

(b)
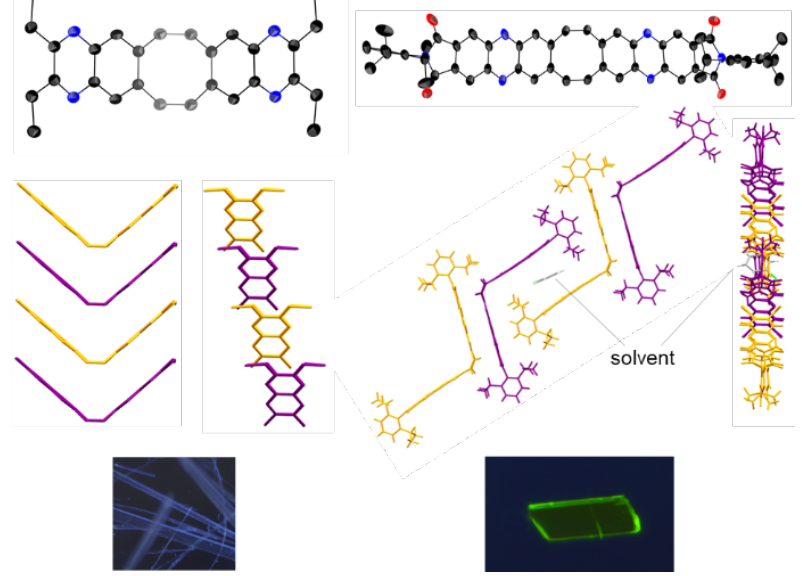

Figure 3. Single-crystal X-ray structure analysis (top) of (a) FLAP1 and (b) FLAP2 (50\% probability for the thermal ellipsoids). Packing structures from different views are shown in the middle. Fluorescent images of each crystal under 365-nm UV light are displayed in the bottom.

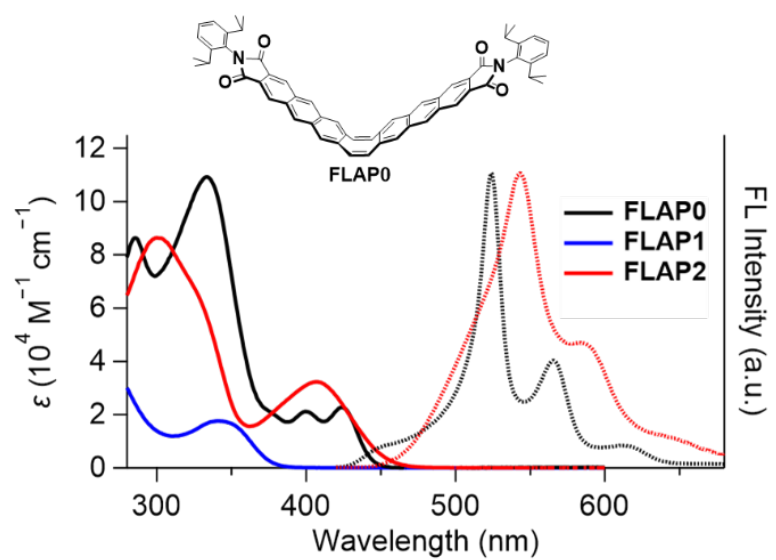

Figure 4. UV-visible absorption and fluorescence (FL) spectra of FLAP0 (black line), FLAP1 (blue line), and FLAP2 (red line) in $\mathrm{CHCl}_{3}$. Excitation wavelength: $340 \mathrm{~nm}$ for FLAP1, and $420 \mathrm{~nm}$ for FLAP0 and FLAP2. The FL spectrum of FLAP1 is not shown due to the very low FL quantum yield $\left(\Phi_{\mathrm{F}}<0.001\right)$.

Although the FLAP1 crystal showed a faint blue fluorescence (Figure 3a, bottom; see the FL spectrum in Figure S4.8), practically no emission was observed in solution. The lowestenergy absorption band does not reach the visible region (Figure 4 , blue line). On the other hand, FLAP2 has a visible absorption band at $408 \mathrm{~nm}$, and a green fluorescence (FL) at $543 \mathrm{~nm}$ was observed in a $\mathrm{CHCl}_{3}$ solution (Figure 4, red lines). Compared with FLAP0, the green FL band has a remarkable shoulder around the short-wavelength region $(450-520 \mathrm{~nm})$. The large Stokes shift of $6100 \mathrm{~cm}^{-1}$ suggested a bent-to-planar conformational change in $\mathrm{S}_{1}$. FL quantum yield $\left(\Phi_{\mathrm{F}}\right)$ and $\mathrm{FL}$ lifetime $\left(\tau_{\mathrm{F}}\right)$ of FLAP2 were determined to be 0.021 and $0.5 \mathrm{~ns}$, respectively. These values are distinct from those of FLAP0 ( $\Phi_{\mathrm{F}}$ $=0.31$ and $\tau_{\mathrm{F}}=9.8 \mathrm{~ns}$ in $\left.\mathrm{CHCl}_{3}\right)$. Lower $\Phi_{\mathrm{F}}$ and shorter $\tau_{\mathrm{F}}$ of FLAP2 can be attributed to fast nonradiative decay, induced by the nitrogen substitution. Solvent dependence was negligible in absorption (Figure S4.2), while a slightly red-shifted fluorescence was observed in DMF (Figure S4.3).

To interpret the large Stokes shift based on the excited-state behavior, constrained TD-DFT calculations were conducted for the model structures, FLAP0' and FLAP2', in which the terminal bulky substituents were replaced by hydrogen atoms for reducing the computational cost (Figure 5). Their $\mathrm{S}_{1}$ energy profiles were delineated by plotting the total energies of the $\mathrm{S}_{1}$ optimized geometries depending on the fixed COT bending angle. As a result, both $S_{1}$ profiles gave double-minimum potentials with the two minima at the bent and planar geometries.
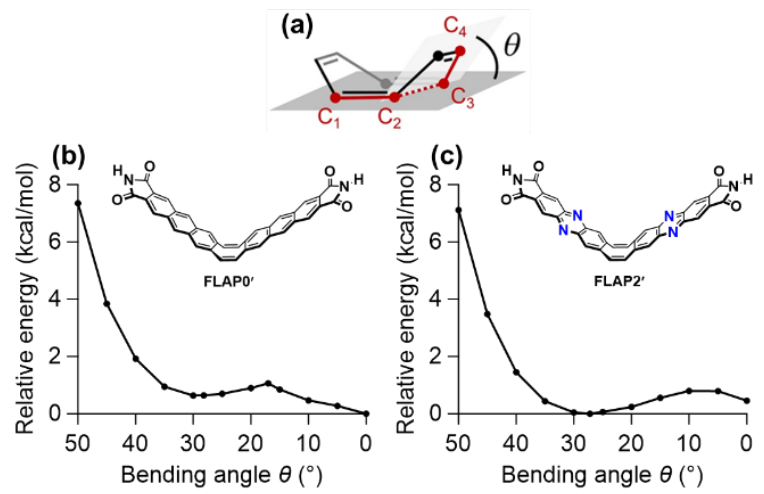

Figure 5. (a) Definition of the COT bending angle. Calculated $\mathrm{S}_{1}$ energy profiles of (b) FLAP0' and (c) FLAP2' in $C_{2 \mathrm{v}}$ symmetry. The constrained structural optimizations in $\mathrm{S}_{1}$ were performed at the TD-PBE0/6-31+G(d) level of theory. 
As for FLAP0', we have previously reported ${ }^{4 a}$ that the small shoulder FL around $450 \mathrm{~nm}$ can be assigned as the emission from the $S_{1}$ local minimum, at which the anthraceneimide moiety is excited in the bent conformation, and that the intense FL at 520 $\mathrm{nm}$ with the large Stokes shift is emitted from the $\mathrm{S}_{1}$ global minimum, at which the effectively $\pi$-conjugated system is formed in the planarized conformation. This explanation can be also applied to FLAP2'. In the $\mathrm{S}_{1}$ profile of FLAP2' calculated at the TD-PBE0/6-31+G(d) level, the relative energy of the bent minimum is slightly lower than that of the planar $S_{1}$ minimum. This is consistent with the more remarkable shoulder FL (450$520 \mathrm{~nm}$ ) of FLAP2' compared with that of FLAP0'. The detailed calculation results are displayed in the Supporting Information, in which the $\mathrm{S}_{1}$ configuration switch during the bent-to-planar conformational change as well as the energetically close $\mathrm{S}_{1}$ configurations at different planar geometries are discussed. (a)

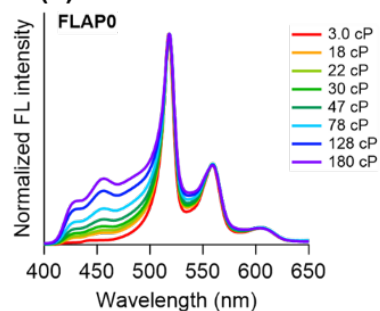

(c)

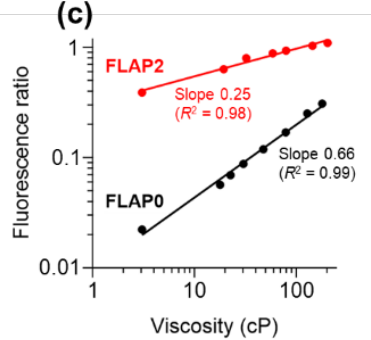

(b)

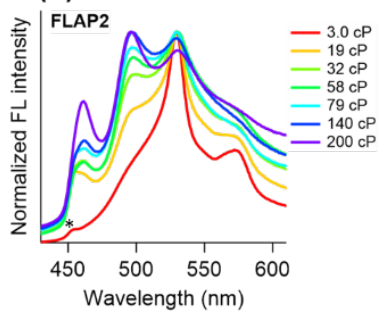

(d)

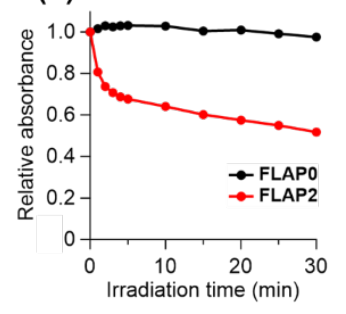

Figure 6. Viscochromism of (a) FLAP0 and (b) FLAP2 in a mixed solvent system of hexadecane/mineral oil. Excitation wavelength: $350 \mathrm{~nm}$ for FLAP0 and $400 \mathrm{~nm}$ for FLAP2. A small band with asterisk $(*)$ was assigned as a Raman peak of the solvent. (c) Förster-Hoffmann plot. The FL ratio values in Figure $6 \mathrm{a}\left(I_{460} / I_{518}\right.$ for FLAP0, black $)$ and Figure $6 \mathrm{~b}\left(I_{497} / I_{530}\right.$ for FLAP2, red) were plotted against the bulk viscosity in the loglog graph. (d) Photodegradation behavior monitored at the 400$\mathrm{nm}$ absorbance (in toluene, concentration: $c a \cdot 10^{-5} \mathrm{M}$ ). Continuous UV irradiation was conducted at $365 \mathrm{~nm}$ with 100 $\mathrm{mW} \mathrm{cm} \mathrm{cm}^{-2}$ intensity.

To endorse the dynamic conformational change in $S_{1}$, we performed the fluorescence measurements of FLAP2 in viscous media. Since the spectral broadening was observed in DMSO/glycerol mixtures (Figure S4.6), we selected the nonpolar mixed solvent system of hexadecane/mineral oil for evaluating the viscosity dependence (Figure 6a-c). Bulk viscosity of the mineral oil itself was determined by a rotational viscometer to be $507 \mathrm{cP}$ at $25^{\circ} \mathrm{C}$, and the Newtonian behavior was confirmed for the mixed solvent system. Namely, the bulk viscosity was constant regardless of the rotation speed of the spindle. As a result, both FLAP0 and FLAP2 showed viscositydependent dual FL properties. With the higher viscosity, the shoulder FL band around $450-500 \mathrm{~nm}$ became increased, indicating partially suppressed bent-to-planar conformational change in $S_{1}$. According to the Förster-Hoffmann plot, the spectral sensitivity to the viscosity change in the 3-200 cP range is relatively higher in FLAP0. In addition, it is worth noting that the photostability of FLAP2 under UV irradiation is much weaker than that of FLAP0 (Figure 6d and S4.5). To elucidate the photodegradation mechanism, we are currently collaborating with laser physicists.

\section{Conclusion}

Nitrogen substitution in the naphthalene and anthraceneimide wings of the photofunctional flapping molecules has been achieved. A key precursor of dibenzo[a,e]COT tetraamine was synthesized in gram scale. Coupling reactions with 1,2-diketone and with dibromo-substituted phthalimide afforded COT-fused quinoxaline dimer and phenazineimide dimer, respectively, as novel nitrogen-embedded flapping systems. While the V-shaped quinoxaline dimer formed a two-fold columnar $\pi$-stacking structure in the crystalline state, the flapping phenazineimide displayed viscosity-dependent dual fluorescence properties. Compared with flapping anthraceneimide, the effect of the nitrogen substitution on the photophysical properties was studied by experiments and theory. Since the phenazine unit plays versatile roles, such as boron-coordinating system, ${ }^{16}$ metal ligands for coordination polymer, ${ }^{17}$ ESIPT (excited-state intramolecular proton transfer) acceptors, ${ }^{18}$ and redox active units showing PCET (proton coupled electron transfer), ${ }^{19}$ the nitrogen-embedded flapping molecules have the potential to endow these roles with dynamic functions. In addition, similar heteroatom-substitution approach will further expand the flapping molecular series, leading to exploration of new functional systems.

\section{Acknowledgement}

This work was partially supported by JST FOREST Program (Grant Number JPXXXX20XX, Japan) and JSPS KAKENHI (Grant Numbers JP21H01917 and JP19J22034, Japan). We also thank Prof. Hideki Yorimitsu (Kyoto University) for his help with high-resolution mass spectrometry measurements.

Supporting Information is available on http://doi.\#\#\#\#\#\#\#\#\#.

\section{References}

1. S. Saito, Molecular Technology: Materials Innovation, Vol. 3 (Eds.: H. Yamamoto, T. Kato), Wiley-VCH, Weinheim, 2019, pp.17-51.

2. D. Shukla, P. Wan, J. Am. Chem. Soc. 1993, 115, 2990.

3. (a) C. Yuan, S. Saito, C. Camacho, S. Irie, I. Hisaki, S. Yamaguchi, J. Am. Chem. Soc. 2013, 135, 8842. (b) C. Yuan, S. Saito, C. Camacho, T. Kowalczyk, S. Irle, S. Yamaguchi, Chem. Eur. J. 2014, 20, 2193.

4. (a) R. Kotani, H. Sotome, H. Okajima, S. Yokoyama, Y. Nakaike, A. Kashiwagi, C. Mori, Y. Nakada, S. Yamaguchi, A. Osuka, A. Sakamoto, H. Miyasaka, S. Saito, J. Mater. Chem. C 2017, 5, 5248. (b) R. Kimura, H. Kuramochi, P. Liu, T. Yamakado, A. Osuka, T. Tahara, S. Saito, Angew. Chem. Int. Ed. 2020, 59, 16430. (c) R. Kimura, H. Kitakado, A. Osuka, S. Saito, Bull. Chem. Soc. Jpn. 2020, 93, 1102.

5. (a) R. Kotani, S. Yokoyama, S. Nobususe, S. Yamaguchi, A. Osuka, H. Yabu, S. Saito, 2020 arXiv:2011.00202v2. (b) T. Yamakado, K. Otsubo, A. Osuka, S. Saito, J. Am. Chem. Soc. 2018, 140, 6245.

6. S. Saito, S. Nobusue, E. Tsuzaka, C. Yuan, C. Mori, M. Hara, T. Seki, C. Camacho, S. Irle, S. Yamaguchi, Nature Commun. 2016, 7, 12094.

7. (a) R. Ayub, O. E. Bakouri, K. Jorner, M. Solà, H. Ottosson, J. Org. Chem. 2017, 82, 6327. (b) J. Toldo, O. E. Bakouri, 
M. Solà, P.-O. Norrby, H. Ottosson, ChemPlusChem 2019, 84, 712. (c) M. Rosenberg, C. Dahlstrand, K. Kilså, Henrik Ottosson, Chem. Rev. 2014, 114, 5379-5425.

8. (a) T. Yamakado, S. Takahashi, K. Watanabe, Y. Matsumoto, A. Osuka, S. Saito, Angew. Chem. Int. Ed. 2018, 57, 5438. (b) M. Hada, S. Saito, S. Tanaka, R. Sato, M. Yoshimura, K. Mouri, K. Matsuo, S. Yamaguchi, M. Hara, Y. Hayashi, F. Röhricht, R. Herges, Y. Shigeta, K. Onda, J. D. Miller, J. Am. Chem. Soc. 2017, 139, 15792. (c) M. Ueda, K. Jorner, Y. M. Sung, T. Mori, Q. Xiao, D. Kim, H. Ottosson, T. Aida, Y. Itoh, Nature Commun. 2017, $8,346$.

9. (a) Z. Zhang, W. Song, J. Su, H. Tian, Adv. Funct. Mater. 2019, 30, 1902803. (b) W. Chen, C.-L. Chen, Z. Zhang, Y.A. Chen, W.-C. Chao, J. Su, H. Tian, P.-T. Chou, J. Am. Chem. Soc. 2015, 137, 8509. (c) Z. Zhang, Y.-S. Wu, K.-C. Tang, C.-L. Chen, J.-W. Ho, J. Su, H. Tian, P.-T. Chou $J$. Am. Chem. Soc. 2015, 137, 8509. (d) G. B. Schuster, S. P. Schmidt, B. G. Dixon, J. Phys. Chem. 1980, 84, 1841.

10. D.-G. Chen, Y. Chen, C.-H. Wu, Y.-A. Chen, M.-C. Chen, J.-A. Lin, C.-Y. Huang, J. Su, H. Tian, P.-T. Chou, Angew. Chem., Int. Ed. 2019, 58, 13297.

11. (a) I. Kawashima, H. Imoto, M. Ishida, H. Furuta, S. Yamamoto, M. Mitsuishi, S. Tanaka, T. Fujii, K. Naka, Angew. Chem. Int. Ed. 2019, 58, 11686. (b) P. B. Karadakov, S. Saito, Angew. Chem. Int. Ed. 2020, 59, 9228.

12. R. Kotani, L. Liu, P. Kumar, H. Kuramochi, T. Tahara, P. Liu, A. Osuka, P. B. Karadakov, S. Saito, J. Am. Chem. Soc. 2020, 142, 14985.

13. (a) A. L. Appleton, S. M. Brombosz, S. Barlow, J. S. Sears, J.-L. Bredas, S. R. Marder, U. H. F. Bunz, Nat. Commun. 2010, 1, 91. (b) M. Takase, T. Narita, W. Fujita, M. S. Asano, T. Nishinaga, H. Benten, K. Yoza, K. Müllen, J. Am. Chem. Soc. 2013, 135, 8031. (c) B. D. Lindner, Y. Zhang, S. Höfle, N. Berger, C. Teusch, M. Jesper, K. I. Hardcastle, X. Qian, U. Lemmer, A. Colsmann, U. H. F. Bunz, M. Hamburger, J. Mater. Chem. C 2013, 1, 5718. (d) Y. Tokimaru, S. Itoh, K. Nozaki, Angew. Chem., Int. Ed. 2015, 54, 7256. (e) H. Yokoi, Y. Hiraoka, S. Hiroto, D. Sakamaki, S. Shu, H. Shinokubo, Nat. Commun. 2015, 6, 8215. (e) Z. Zhang, Q. Zhang, Mater. Chem. Front. 2020, 4, 3419.

14. P. A. Wender, A. B. Lesser, L. E. Sirois, Angew. Chem. Int. Ed. 2012, 51, 2736.

15. J. K. Laha, K. S. S. Tummalapalli, A. Gupta, Eur. J. Org. Chem. 2013, 8330.

16. Y. Min, C. Dou, H. Tian, Y. Geng, J. Liu, L. Wang, Angew. Chem. Int. Ed. 2018, 57, 2000.

17. M. Munakata, S. Kitagawa, N. Ujimaru, M. Nakamura, M. Maekawa, H. Matsuda, Inorg. Chem. 1993, 32, 826.

18. J. Piechowska, K. Virkki, B. Sadowski, H. Lemmetyinen, N. V. Tkachenko, D. T. Gryko, J. Phys. Chem. A 2014, 118, 144.

19. (a) H. Xie, Y. Wu, T. Liu, F. Wang, B. Chen, B. Liang, Applied Energy, 2020, 259, 114119. (b) M. N. Jackson, M. L. Pegis, Y. Surendranath, ACS Cent. Sci. 2019, 5, 831. 


\section{Graphical Abstract}

Nitrogen-Substitution in the Flapping Wings of Cyclooctatetraene-Fused Molecules

Kensuke Suga, Takuya Yamakado, and Shohei Saito*

$<$ Summary $>$

Nitrogen atoms are embedded in the acene wings of the flapping molecules bearing a cyclooctatetraene ring at its center, and the nitrogen substitution effects are studied. V-shaped flapping quinoxaline molecules formed a columnar $\pi$-stacking structure in the crystalline phase. Flapping phenazineimide showed a viscosity-dependent dual fluorescence, originating from the double-minimum energy potential in the lowest singlet excited state.

$<$ Diagram $>$

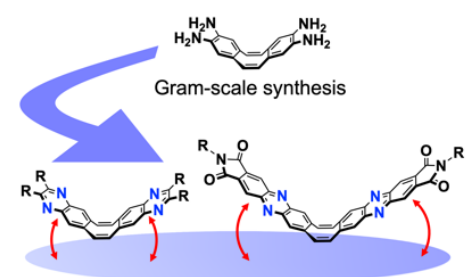

Nitrogen-Embedded FLAPs

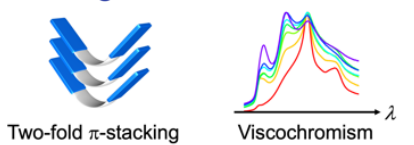




\section{Supporting Information}

\section{Nitrogen-Substitution in the Flapping Wings of Cyclooctatetraene-}

\section{Fused Molecules}

Kensuke Suga, ${ }^{1}$ Takuya Yamakado, ${ }^{1}$ and Shohei Saito ${ }^{* 1}$

${ }^{[1]}$ Graduate School of Science, Kyoto University, Kitashirakawa Oiwake-cho, Sakyo-ku, Kyoto 606-8502 (Japan)

\section{Corresponding Author}

s_saito@kuchem.kyoto-u.ac.jp

\section{Contents}

1. General information S2

2. Synthesis of compounds S3-S5

3. Characterization of new compounds. S6-S11

4. Photophysical properties. S12-S18

5. X-ray crystallographic analysis S19-S21

6. Theoretical calculations S22-S36

7. Supporting references. S37 


\section{General information}

\section{Synthesis}

All reagents and solvents were of commercial grade and were used without further purification unless otherwise noted. Tetrahydrofuran (THF) and dioxane were dried using Glass Contour solvent purification system. Thin layer chromatography (TLC) was carried out on aluminum sheets coated with silica gel $60 \mathrm{~F}_{254}$ (Merck). Column chromatography was performed on Wakoge ${ }^{\circledR} \mathrm{C}-300$ and C-400. ${ }^{1} \mathrm{H}$ and ${ }^{13} \mathrm{C}$ NMR spectra were recorded on a JEOL ECA-600 (600 MHz for ${ }^{1} \mathrm{H}$ and $151 \mathrm{MHz}$ for $\left.{ }^{13} \mathrm{C}\right)$ spectrometer. Chemical shifts were reported in ppm relative to the residual solvents as internal standards $\left(\mathrm{CHCl}_{3}: \delta\right.$ $=7.26 \mathrm{ppm}$ for ${ }^{1} \mathrm{H}$ and $\delta=77.16 \mathrm{ppm}$ for ${ }^{13} \mathrm{C}$; DMSO: $\delta=2.50 \mathrm{ppm}$ for ${ }^{1} \mathrm{H}$ and $\delta=39.52 \mathrm{ppm}$ for ${ }^{13} \mathrm{C}$ ). High-resolution atmospheric-pressure-chemical-ionization time-of-flight mass spectrometry (HR-APCI-TOF-MS) was performed with a BRUKER micrOTOF model. Compounds $\mathbf{1}^{[\mathrm{S} 1]}$ and $\mathbf{4}^{[\mathrm{S} 2]}$, and FLAP0 ${ }^{[\mathrm{S} 3]}$ were synthesized according to the reported methods.

\section{Photophysical measurements}

UV-visible absorption spectra were recorded on a Shimadzu UV-3600 spectrometer. Steady-state fluorescence and excitation spectra were recorded on a JASCO FP-8500 spectrofluorometer. Absolute fluorescence quantum yields were determined on a HAMAMATSU C9920-02S apparatus. Fluorescence lifetimes were measured on a compact fluorescence lifetime spectrometer Hamamatsu Photonics QuantaurusTau C11367 (Tables S4.1 and S4.2). Low temperature measurements were performed using a Unisoku CoolSpeK UV USP-203-B cryostat. All measurements were conducted using a 10-mm square quartz cell, unless otherwise noted. Bulk viscosity of the mixed system of hexadecane/mineral oil was measured at $25^{\circ} \mathrm{C}$ by using a DV2T touch screen viscometer (AMETEK Brookfield). Fluorescence microscope images were taken with a digital camera (Leica DFC450 C) connected to an upright microscope (Leica DM2700 P). To evaluate the photostability of compounds, a UV-LED light source (HAMATSU LIGHTSOURCETM LC-L1V3 equipped with C11924-111 and L11921$400, \lambda=365 \mathrm{~nm}, 100 \mathrm{~mW} \mathrm{~cm}^{-2}$ ) was used.

\section{X-ray crystallography}

X-ray crystallographic data of FLAP1 and FLAP2 were collected at $-180{ }^{\circ} \mathrm{C}$ with a Rigaku XtaLAB P200 diffractometer equipped with a copper MicroMax007HF generator, VariMax-Cu optics, and a PILATUS 200K twodimensional detector. The structure was solved by direct method SHELXT ${ }^{[\mathrm{S} 4]}$ and refined by SHELXL ${ }^{[\mathrm{S} 5]}$ programs. All nonhydrogen atoms were refined anisotropically and all hydrogen atoms were placed using AFIX instructions. Crystallographic data have been deposited in the Cambridge Crystallographic Data Center with the CCDC numbers of 2081297 (FLAP1) and 2081298 (FLAP2). 


\section{Synthesis of compounds}

\section{Synthesis of 2}

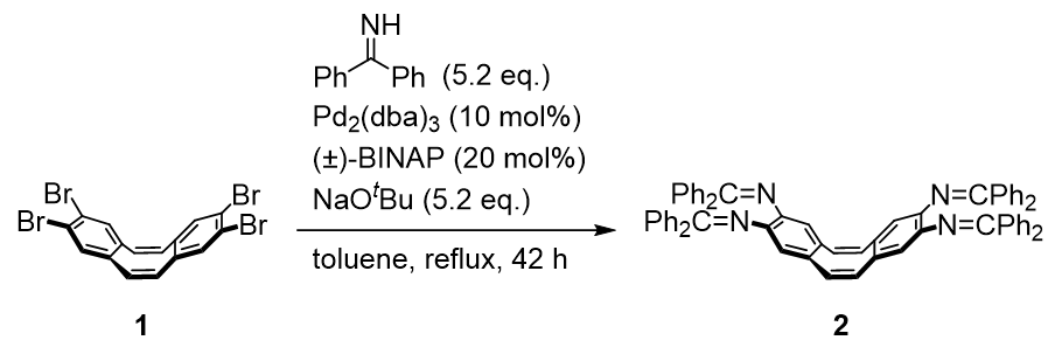

$\mathrm{Pd}_{2}(\mathrm{dba})_{3}(630 \mathrm{mg}, 690 \mu \mathrm{mol})$ and $( \pm)$-BINAP $(920 \mathrm{mg}, 1.4 \mathrm{mmol})$ were placed in a two-necked flask, and the flask was purged with argon. The mixture was dissolved with distilled toluene $(170 \mathrm{~mL})$, stirred at $110{ }^{\circ} \mathrm{C}$ for $30 \mathrm{~min}$, and the solution was cooled to room temperature. Compound $1(3.5 \mathrm{~g}, 6.7 \mathrm{mmol}), \mathrm{NaO}{ }^{t} \mathrm{Bu}(3.4 \mathrm{~g}, 35 \mathrm{mmol})$, and benzophenone imine $(5.9$ $\mathrm{mL}, 35 \mathrm{mmol}$ ) were added to the solution, and the mixture was refluxed for $42 \mathrm{~h}$. The resulting mixture was cooled to room temperature, diluted with an excess amount of $\mathrm{CHCl}_{3}$, and filtered through a Celite pad. The filtrate was evaporated to obtain a black residue. The residue was purified by silica gel column chromatography $\left(\mathrm{CH}_{2} \mathrm{Cl}_{2} /\right.$ hexane/Et $\left.3 \mathrm{~N}, 90: 10: 1\right)$ to obtain crude product as orange solid. Through continuous precipitation from $\mathrm{CH}_{2} \mathrm{Cl}_{2} / \mathrm{MeOH}$ and filtration, pure compound 2 was obtained as a yellow solid (5.0 g, 81\%). ${ }^{1} \mathrm{H}$ NMR ( $\left.\mathrm{CDCl}_{3}, 600 \mathrm{MHz}\right): \delta(\mathrm{ppm}) 7.66(\mathrm{~d}, J=7.2 \mathrm{~Hz}, 8 \mathrm{H}), 7.39$ (t, $\left.J=7.8 \mathrm{~Hz}, 4 \mathrm{H}\right), 7.32$ (t, $J=7.8 \mathrm{~Hz}, 8 \mathrm{H}), 7.29$ (t, $J=7.2 \mathrm{~Hz}, 4 \mathrm{H}), 7.19$ (t, $J=7.5 \mathrm{~Hz}, 8 \mathrm{H}), 6.84(\mathrm{~d}, J=7.8 \mathrm{~Hz}, 8 \mathrm{H}), 6.21(\mathrm{~s}, 4 \mathrm{H}), 6.10(\mathrm{~s}, 4 \mathrm{H}) ;{ }^{13} \mathrm{C}$ NMR $\left(\mathrm{CDCl}_{3}, 151 \mathrm{MHz}\right) \delta(\mathrm{ppm}) 167.43,140.35,140.18,136.83,132.53,132.44,130.37,129.54,129.47,128.78,128.03$, 127.94, and 121.94; HR-APCI-TOF-MS $(m / z)$ found 920.3841, calcd for $\mathrm{C}_{68} \mathrm{H}_{48} \mathrm{~N}_{4}: 920.3873[M]^{+}$.

\section{Synthesis of 3}

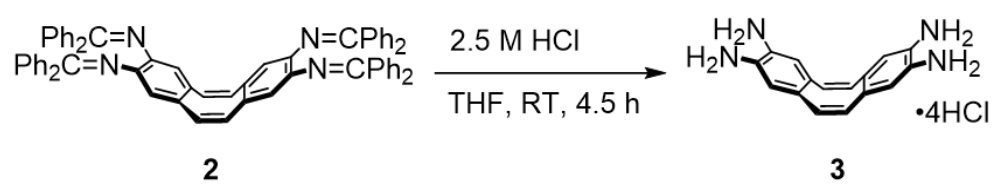

Compound $2(5.0 \mathrm{~g}, 5.4 \mathrm{mmol})$ was placed in a two-necked flask, and the flask was purged with argon. To the flask, anhydrous THF $(330 \mathrm{~mL})$ and an aqueous solution of $\mathrm{HCl}(2.5 \mathrm{M}, 11 \mathrm{~mL})$ were added, and the reaction mixture was stirred at $25^{\circ} \mathrm{C}$ for $4.5 \mathrm{~h}$. The resulting suspension was collected by filtration under nitrogen flow and dried under vacuum, providing 3 as a white solid ( $2.5 \mathrm{~g}$, quant.). The product was used for the next step without further purification and kept under argon atmosphere at $-30{ }^{\circ} \mathrm{C}$ because of the poor stability in air. ${ }^{1} \mathrm{H}$ NMR (DMSO- $d_{6}, 600 \mathrm{MHz}$ ): $\delta$ (ppm) 8.4-6.9 (very broad), 6.67 $(\mathrm{s}, 4 \mathrm{H}), 6.55(\mathrm{~s}, 4 \mathrm{H}) .{ }^{13} \mathrm{C}$ NMR (DMSO- $\left.d_{6}, 151 \mathrm{MHz}\right) \delta(\mathrm{ppm}) 132.14,130.00,128.58$, and 120.8-120.0 (br). 


\section{Synthesis of FLAP1}

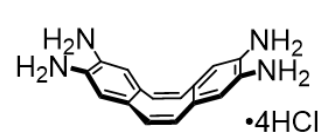

3

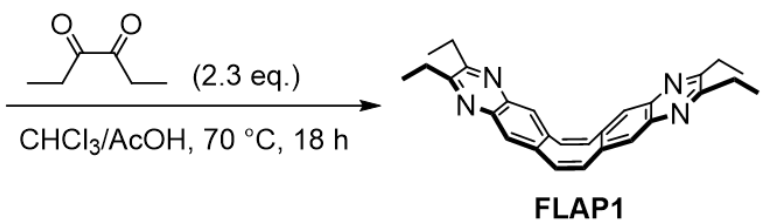

FLAP1

Compound 3 (150 mg, $370 \mathrm{mmol}$ ) was placed in a Schlenk flask, and the flask was purged with argon. To the flask, $\mathrm{CHCl}_{3}$ (4.5 mL, $\mathrm{N}_{2}$ bubbled), acetic acid $\left(2.7 \mathrm{~mL}, \mathrm{~N}_{2}\right.$ bubbled), and 3,4-hexanedione $(0.10 \mathrm{~mL}, 830 \mathrm{mmol})$ were added, and the resulting suspension was stirred at $70{ }^{\circ} \mathrm{C}$ for $18 \mathrm{~h}$. The resulting mixture was cooled to room temperature and extracted with $\mathrm{CHCl}_{3}$. The organic layer was washed with an aqueous $\mathrm{NaHCO}_{3}$ solution, dried over anhydrous $\mathrm{Na}_{2} \mathrm{SO}_{4}$, and then evaporated. The crude product was purified by recrystallization from THF/MeOH to obtain pure FLAP1 as a white solid (106 mg, 68\%). ${ }^{1} \mathrm{H} \mathrm{NMR}\left(\mathrm{CDCl}_{3}, 600 \mathrm{MHz}\right): \delta(\mathrm{ppm}) 7.74$ (s, 4H), 7.10 (s, 4H), 2.96 (q, $\left.J=7.2 \mathrm{~Hz}, 8 \mathrm{H}\right), 1.33(\mathrm{t}, J=7.2 \mathrm{~Hz}, 12 \mathrm{H})$; ${ }^{13} \mathrm{C}$ NMR (CDCl $\left.3,151 \mathrm{MHz}\right): \delta(\mathrm{ppm}) 157.73,139.93,137.82,133.21,128.17,28.75$, and 12.57; HR-APCI-TOF-MS $(\mathrm{m} / \mathrm{z})$ found 420.2306, calcd for $\mathrm{C}_{28} \mathrm{H}_{28} \mathrm{~N}_{4}: 420.2308[M]^{+}$.

\section{Synthesis of FLAP2}

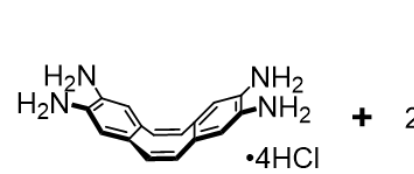

3

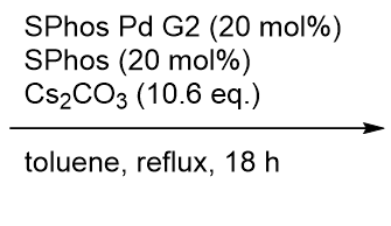

4

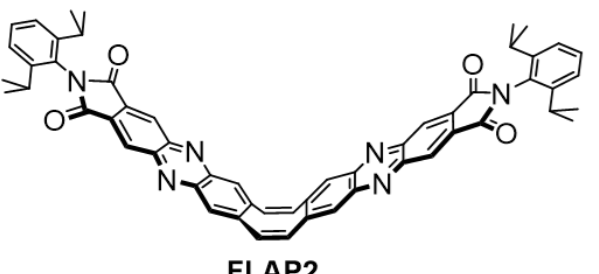

FLAP2

Compound 4 (130 mg, $290 \mu \mathrm{mol})$, SPhos Pd G2 (21 mg, $29 \mu \mathrm{mol})$, SPhos (12 mg, $29 \mu \mathrm{mol}), \mathrm{Cs}_{2} \mathrm{CO}_{3}(510 \mathrm{mg}, 1.6$ $\mathrm{mmol})$, and compound $\mathbf{3}(60 \mathrm{mg}, 150 \mu \mathrm{mol})$ were placed in a Schlenk flask, and the flask was purged with argon. To the flask, distilled toluene $(2.3 \mathrm{~mL})$ was added, then the resulting suspension was refluxed for $18 \mathrm{~h}$. The resulting mixture was cooled to room temperature and filtrated through a Celite ${ }^{\circledR}$ pad. The resulting solution was evaporated under reduced pressure, and the residue was purified by a flash silica-gel column chromatography (eluent: $\mathrm{CHCl}_{3} / \mathrm{EtOAc}(1: 1)$ ) for two times. The crude product was reprecipitated from $\mathrm{CHCl}_{3} / \mathrm{MeOH}$ for three times to obtain pure FLAP2 as a yellow solid (35.4 mg, 28\%). ${ }^{1} \mathrm{H}$ NMR (CDCl $3,600 \mathrm{MHz}): \delta(\mathrm{ppm}) 8.78(\mathrm{~s}, 4 \mathrm{H}), 8.19(\mathrm{~s}, 4 \mathrm{H}), 7.49$ (t, $J=7.8 \mathrm{~Hz}, 2 \mathrm{H}), 7.38(\mathrm{~s}, 4 \mathrm{H}), 7.32(\mathrm{~d}, J=7.8 \mathrm{~Hz}, 4 \mathrm{H})$, $2.73(\mathrm{sep}, J=6.8 \mathrm{~Hz}, 4 \mathrm{H}), 1.18$ (d, $J=6.8 \mathrm{~Hz}, 24 \mathrm{H}) ;{ }^{13} \mathrm{C} \mathrm{NMR}\left(\mathrm{CDCl}_{3}, 151 \mathrm{MHz}\right): \delta(\mathrm{ppm}) 166.66,146.96,145.52,143.37$, 141.44, 133.49, 131.01, 130.64, 129.65, 127.83, 126.97, 124.27, 29.60, and 24.15; HR-APCI-TOF-MS $(\mathrm{m} / \mathrm{z})$ found 866.3607, calcd for $\mathrm{C}_{56} \mathrm{H}_{46} \mathrm{~N}_{6} \mathrm{O}_{4}: 866.3586[M]^{-}$. 


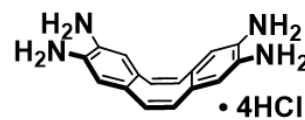

3

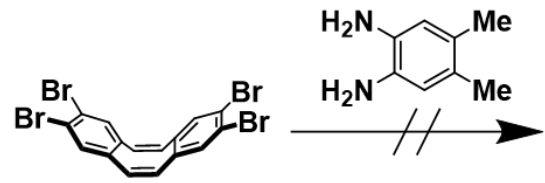

1

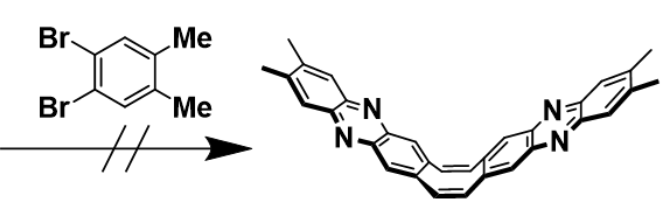

5

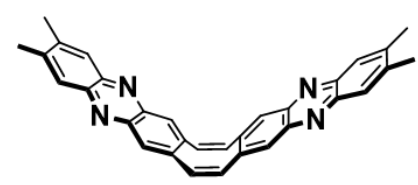

5

Figure S2.1. Schemes for the synthesis of $\mathbf{5}$. We attempted to synthesize 5 by applying the same reaction conditions in the synthesis of FLAP2. The coupling partners of $\mathbf{3}$ and 1,2-dibromo-4,5-dimethylbenzene, as well as $\mathbf{1}$ and 4,5-dimethylbenzene1,2-diamine, were used for the reaction, but 5 was not obtained in this reaction condition probably because the reaction stopped halfway due to the poor solubility. 
3. Characterization of new compounds

\section{1. ${ }^{1} \mathrm{H}$ and ${ }^{13} \mathrm{C}$ NMR spectroscopy}

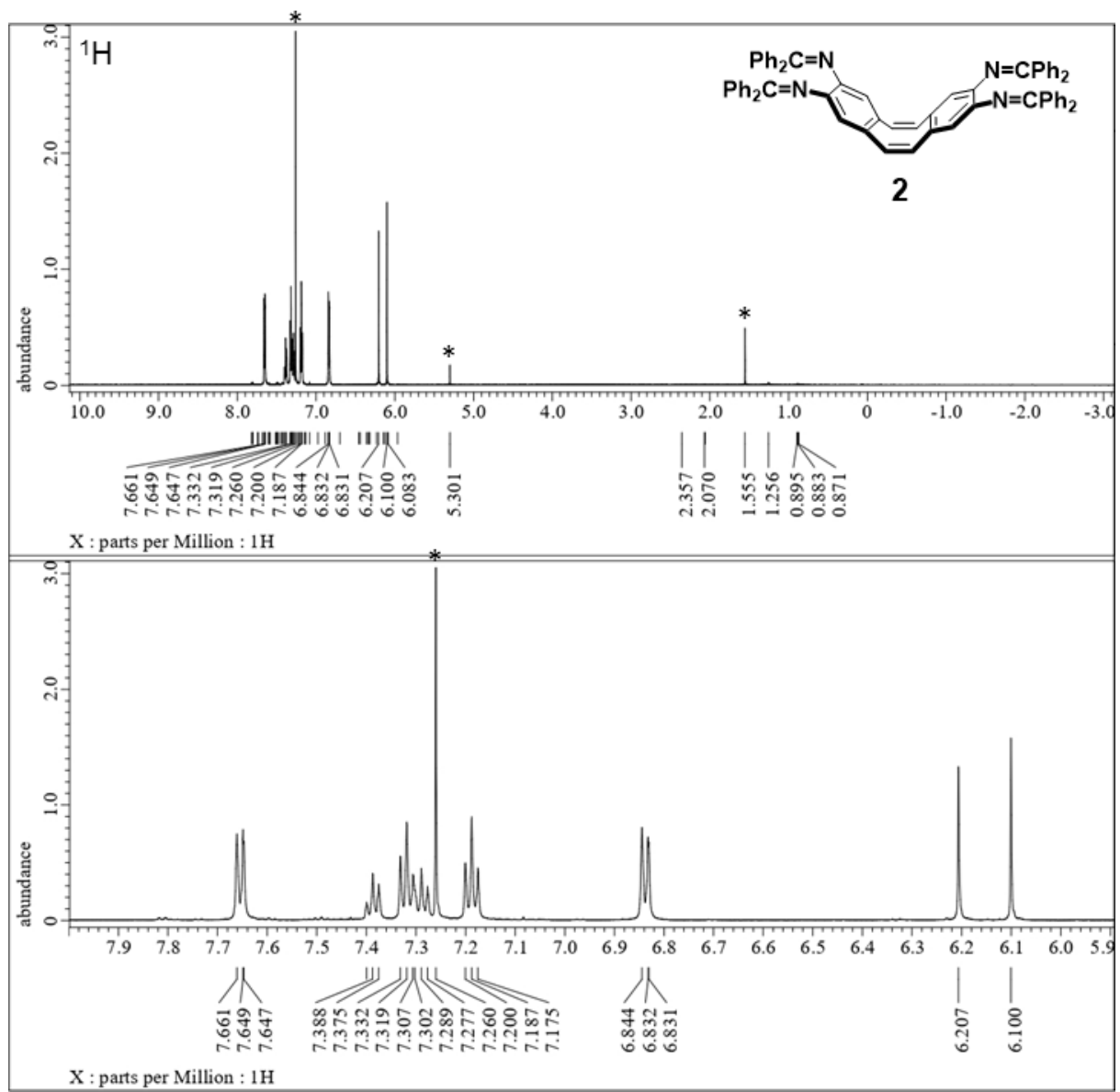

$\mathrm{X}$ : parts per Million : $1 \mathrm{H}$

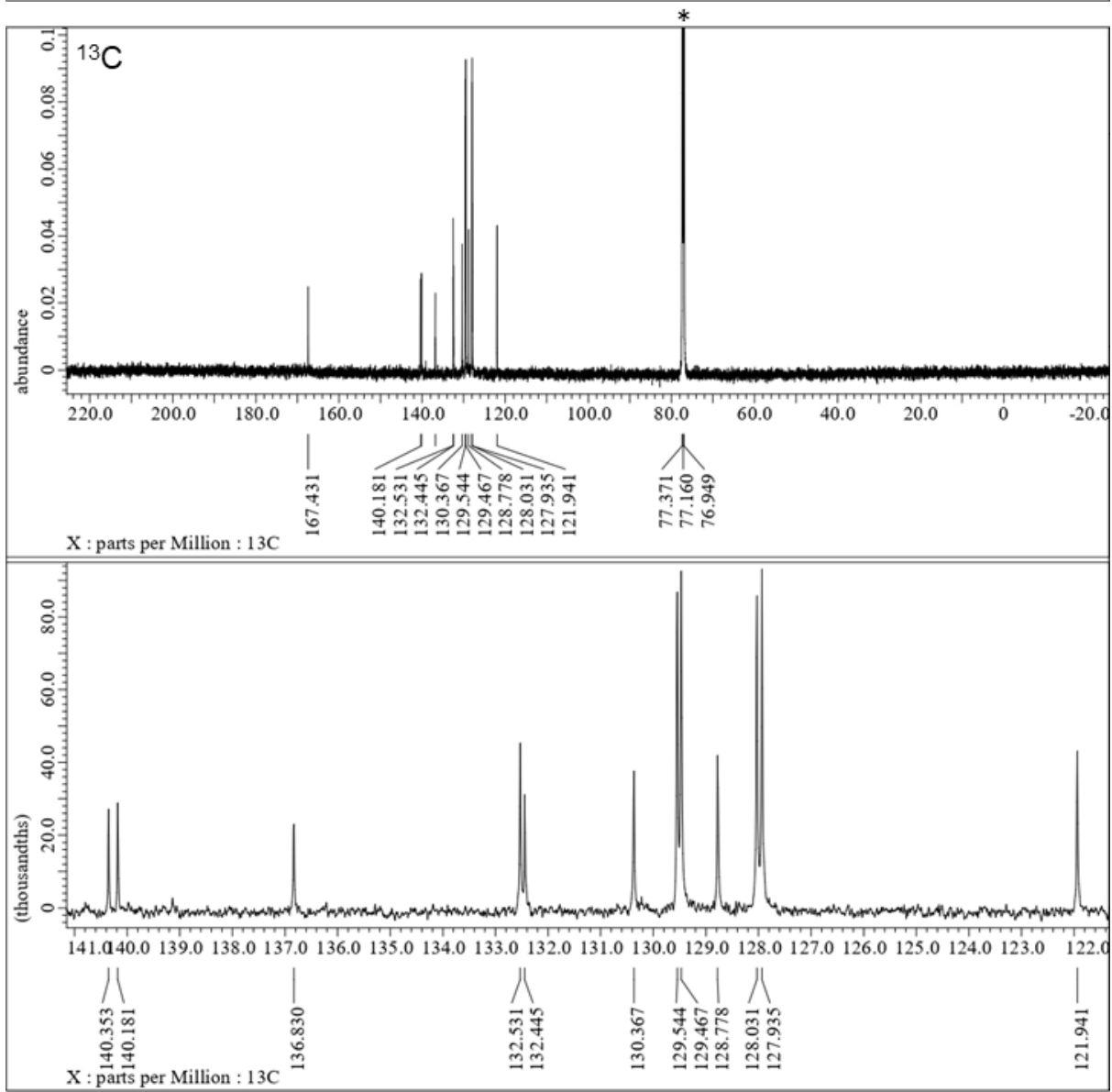

Figure S3.1. ${ }^{1} \mathrm{H}$ NMR (top) and ${ }^{13} \mathrm{C}$ NMR (bottom) spectra of $\mathbf{2}$ in $\mathrm{CDCl}_{3}$. Signals marked with asterisk ( ${ }^{\star}$ ) indicate residual solvents. 

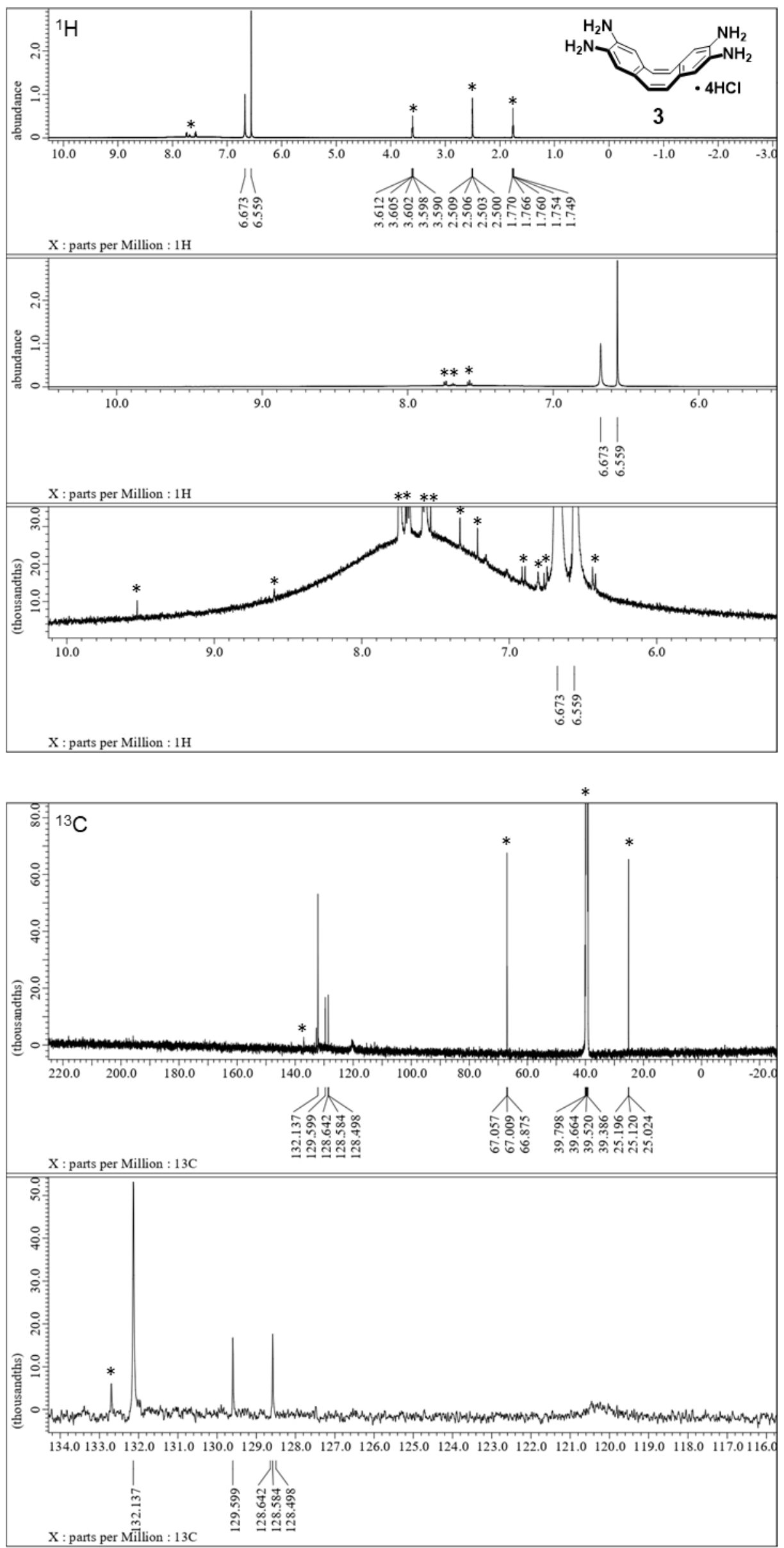

Figure S3.2. ${ }^{1} \mathrm{H}$ NMR (top) and ${ }^{13} \mathrm{C}$ NMR (bottom) spectra of $\mathbf{3}$ in DMSO- $d_{6}$. Signals marked with asterisk ( ${ }^{*}$ ) indicate residual solvents and impurities. 

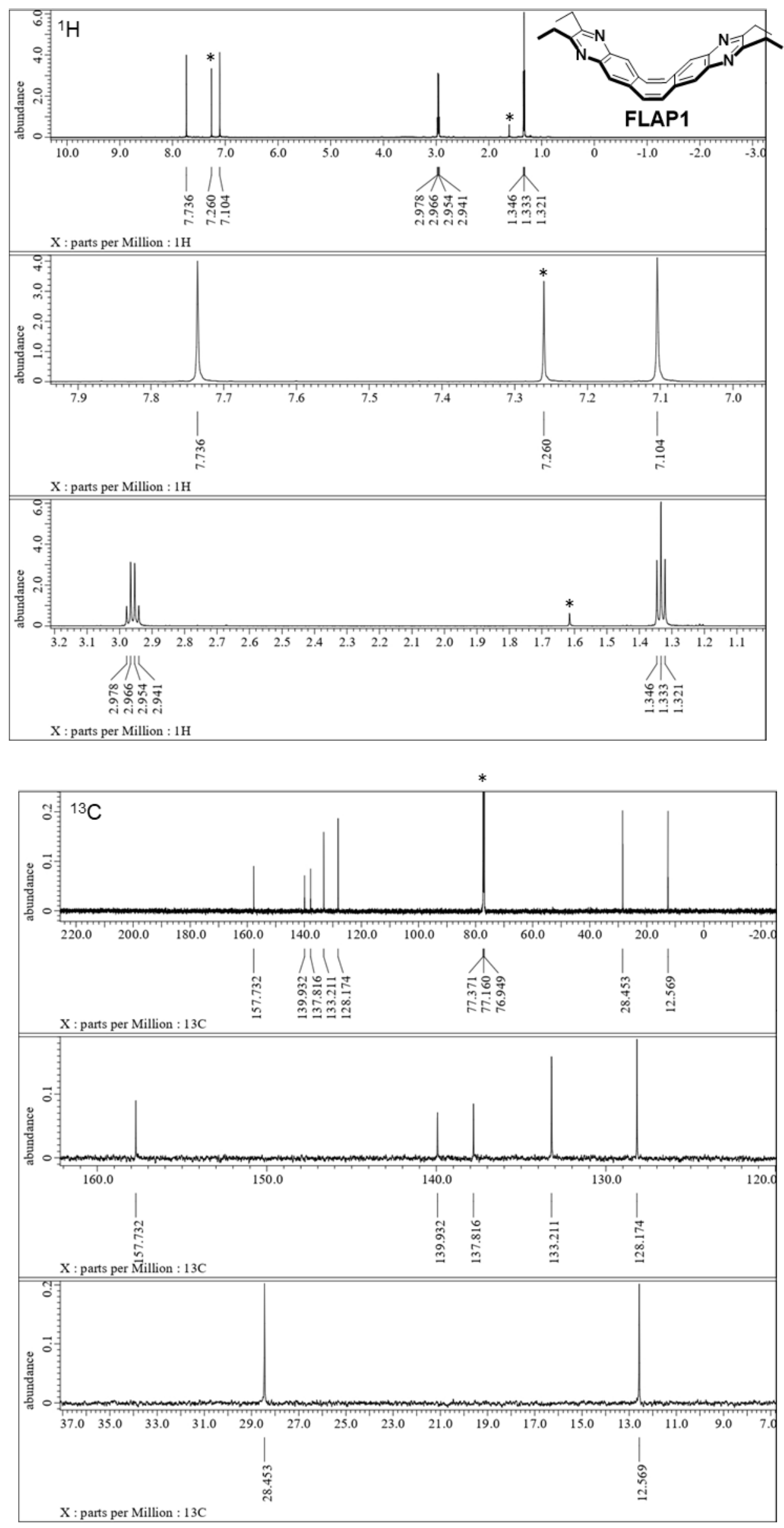

Figure S3.3. ${ }^{1} \mathrm{H}$ NMR (top) and ${ }^{13} \mathrm{C}$ NMR (bottom) spectra of FLAP1 in $\mathrm{CDCl}_{3}$. Signals marked with asterisk $\left({ }^{*}\right)$ indicate residual solvents. 

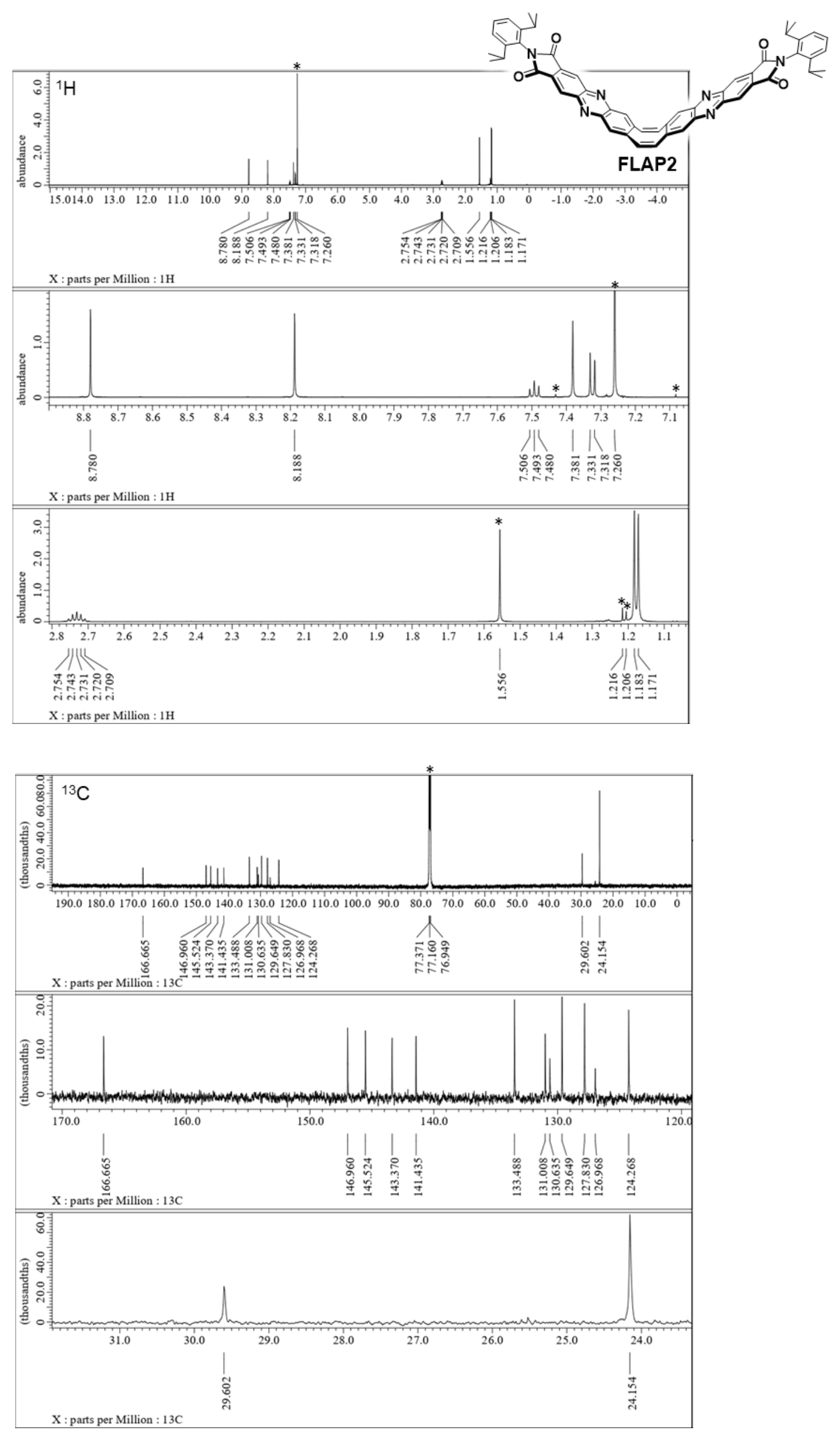

Figure S3.4. ${ }^{1} \mathrm{H}$ NMR (top) and ${ }^{13} \mathrm{C}$ NMR (bottom) spectra of FLAP2 in $\mathrm{CDCl}_{3}$. Signals marked with asterisk $\left({ }^{*}\right)$ indicate residual solvents and impurities. 


\subsection{High resolution mass spectrometry}

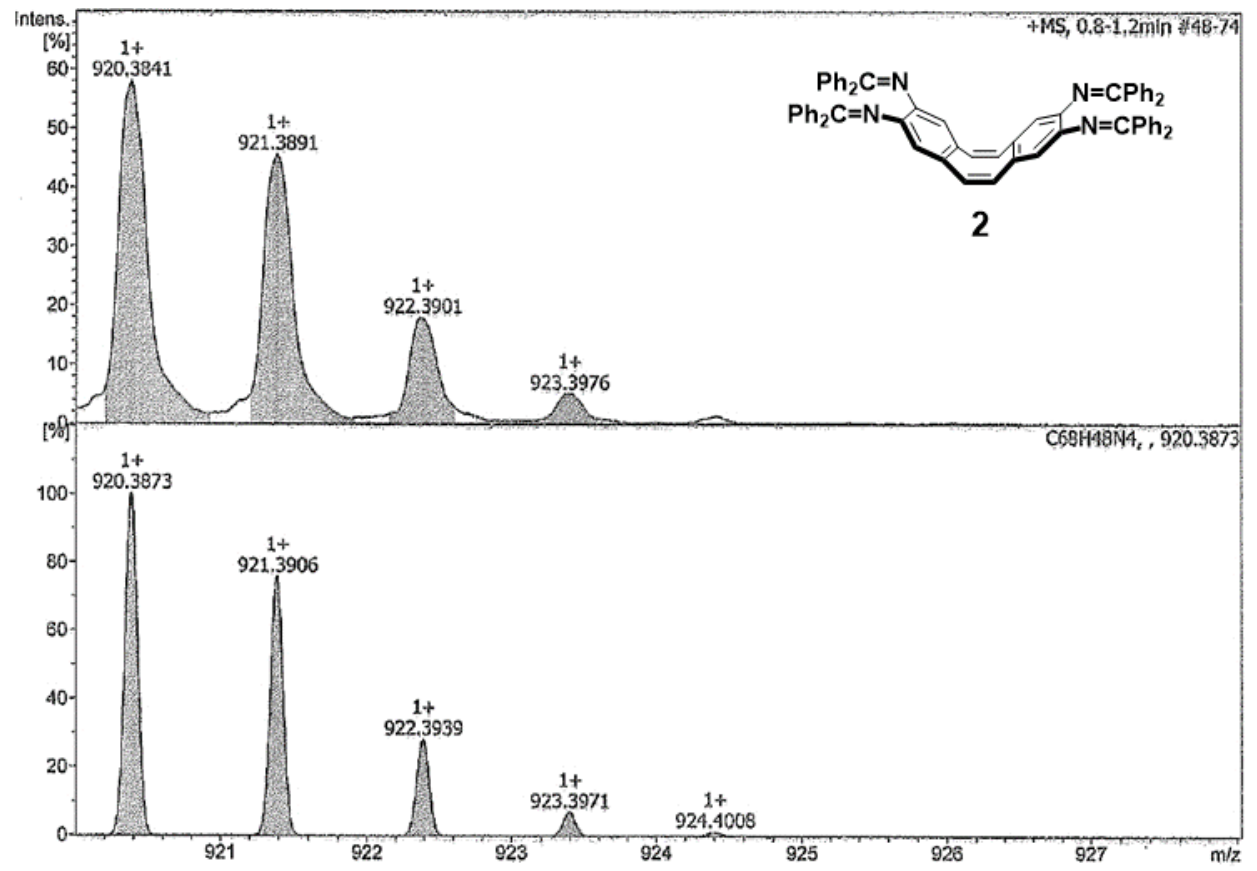

Figure S3.5. HR-APCI-TOF-MS (positive mode) of 2 (top: observed, bottom: simulated).

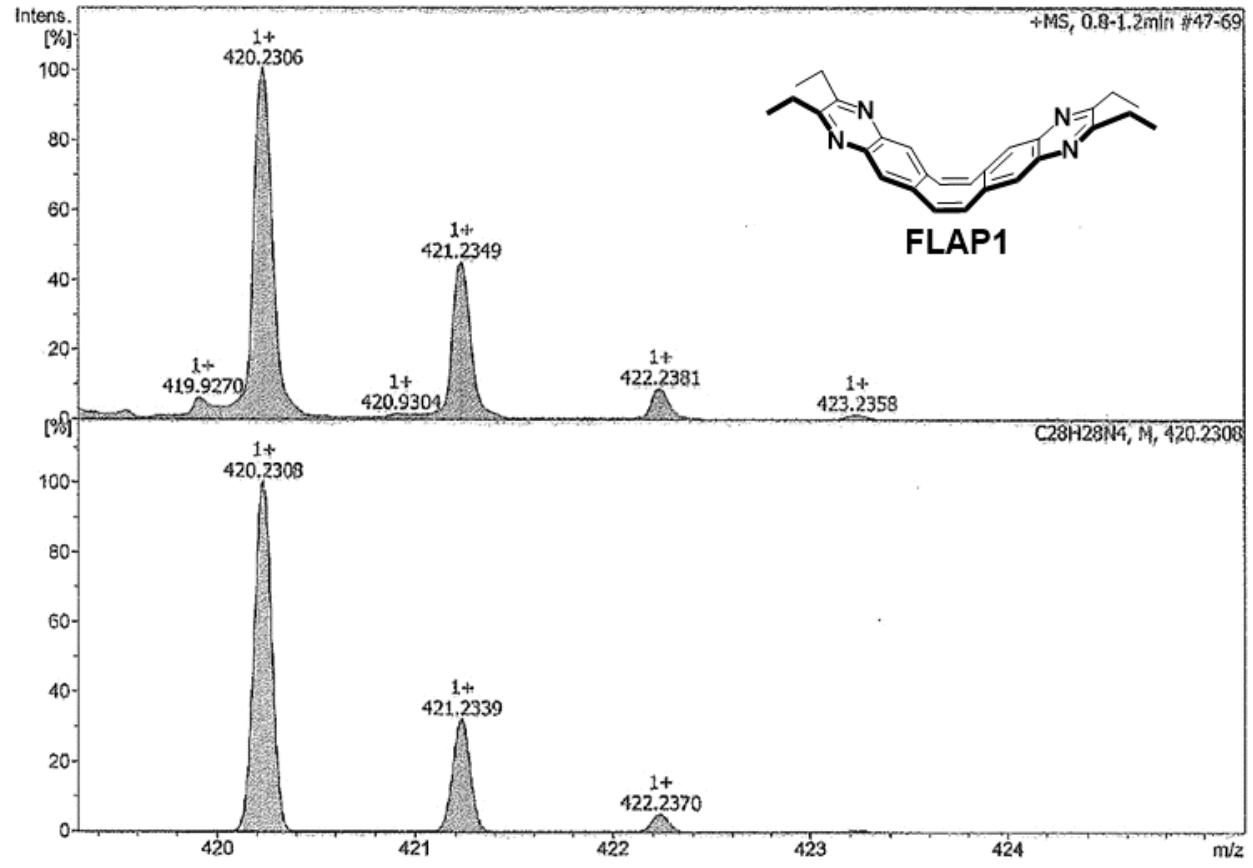

Figure S3.6. HR-APCI-TOF-MS (positive mode) of FLAP1 (top: observed, bottom: simulated). 


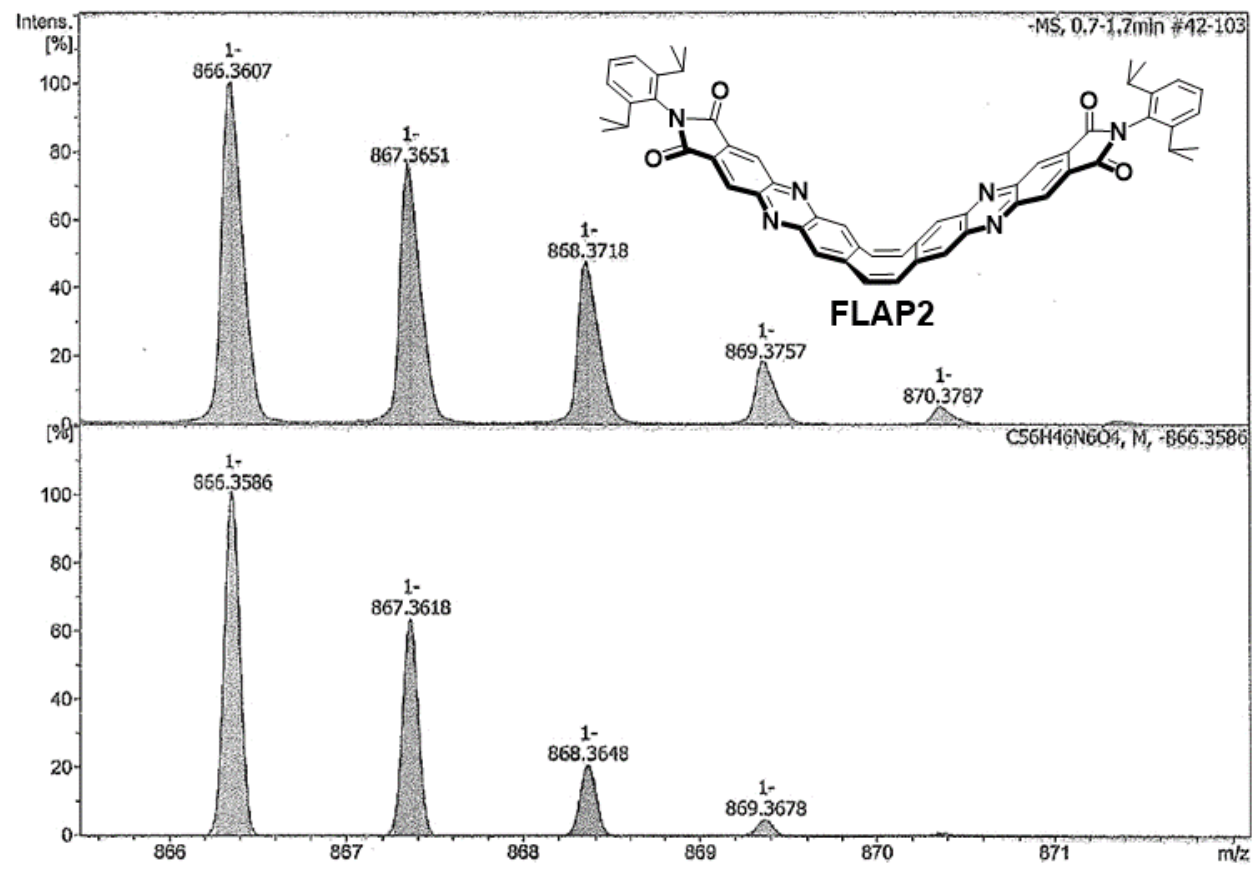

Figure S3.7. HR-APCI-TOF-MS (negative mode) of FLAP2 (top: observed, bottom: simulated). 


\section{Photophysical properties}

\subsection{UV-visible absorption and fluorescence spectra}
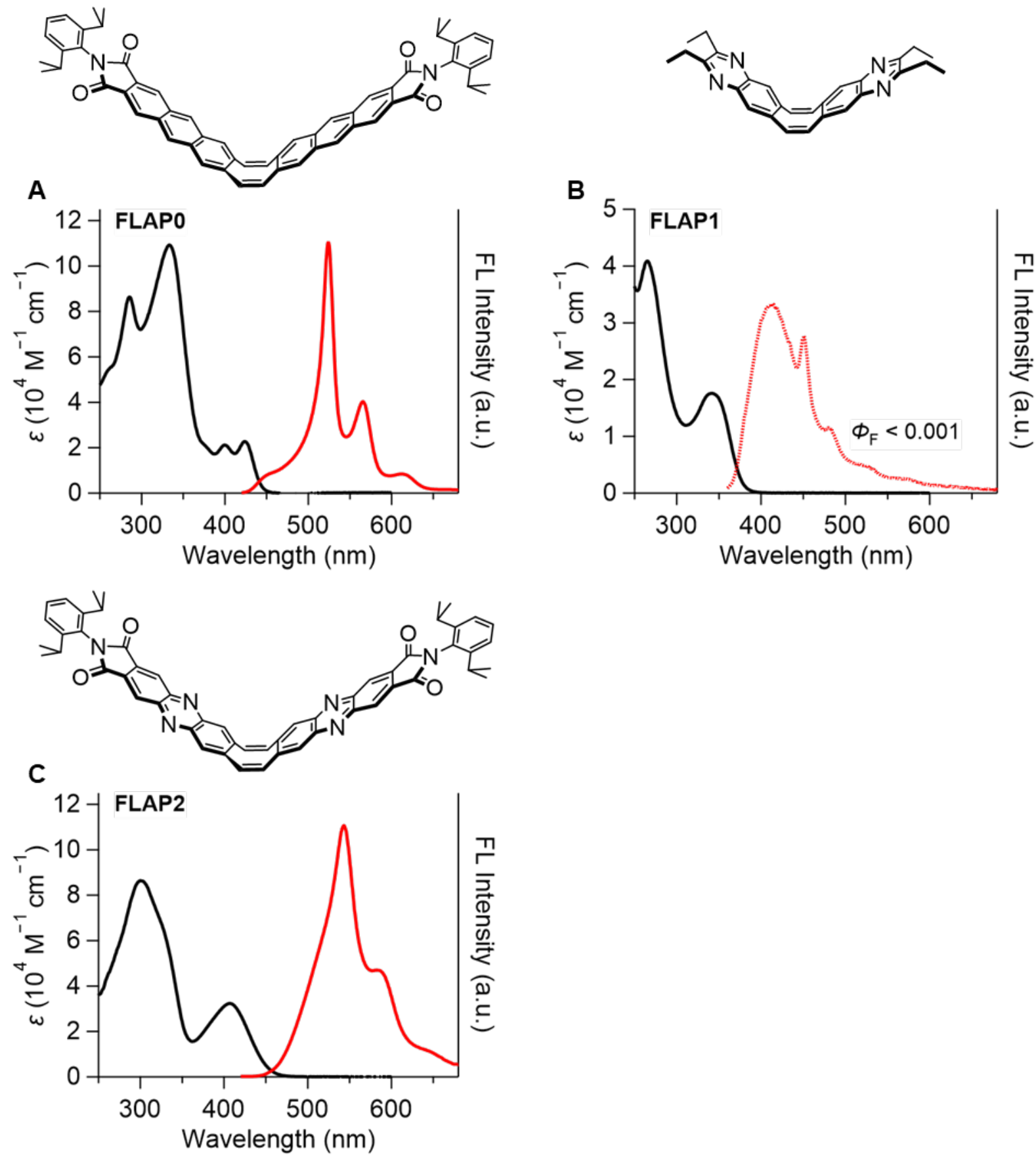

Figure S4.1. UV-visible absorption (black line) and fluorescence (red line) spectra of (A) FLAP0, (B) FLAP1, (C) FLAP2 in $\mathrm{CHCl}_{3}$ (excitation wavelength $\lambda_{\mathrm{ex}}=340 \mathrm{~nm}$ for FLAP1, $\lambda_{\mathrm{ex}}=400 \mathrm{~nm}$ for FLAP0 and FLAP2). 


\subsection{Solvent dependence}
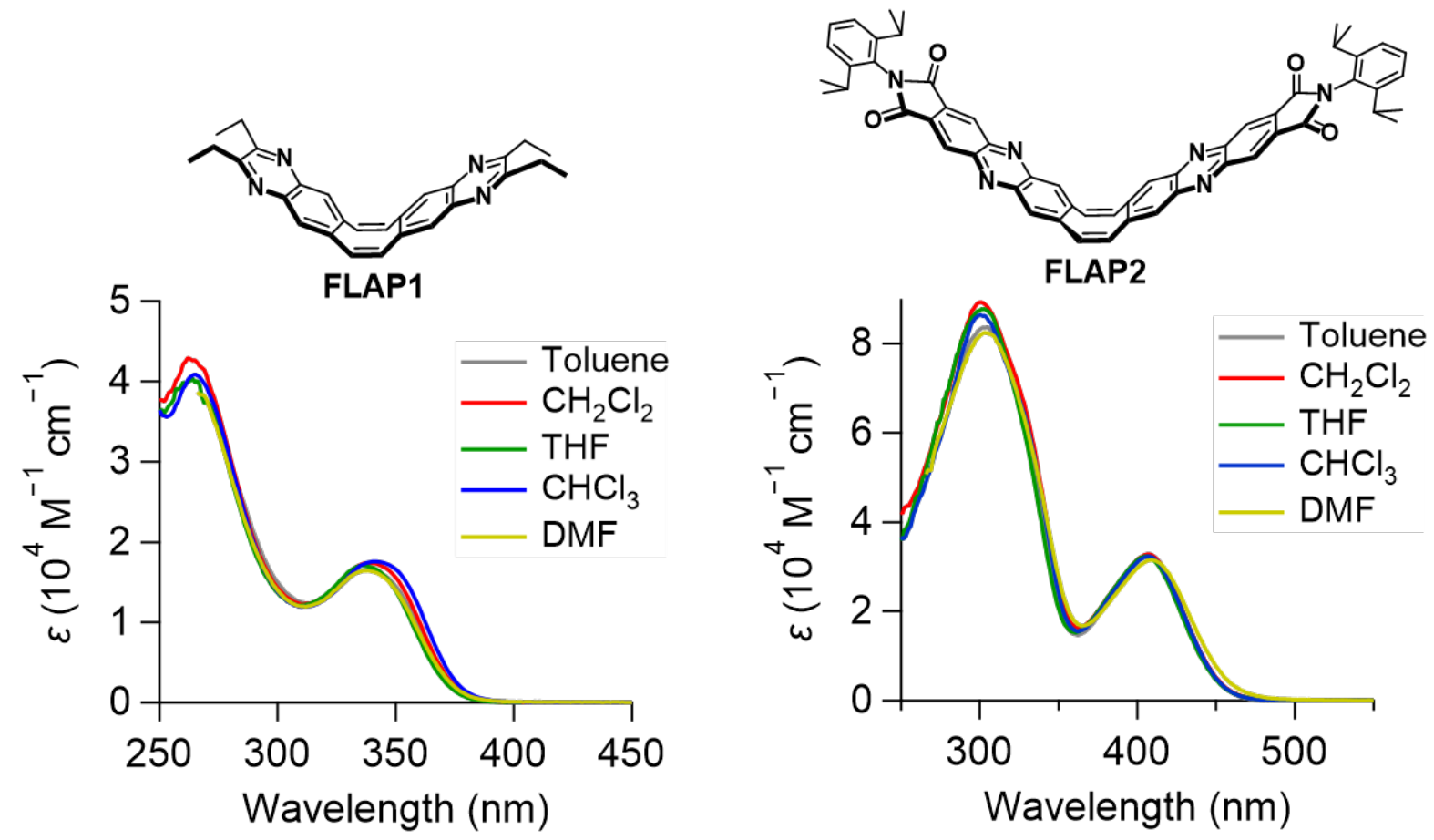

Figure S4.2. UV-visible absorption spectra of FLAP1 and FLAP2 in various solvents with the concentration of $8 \times 10^{-6} \mathrm{M}$.

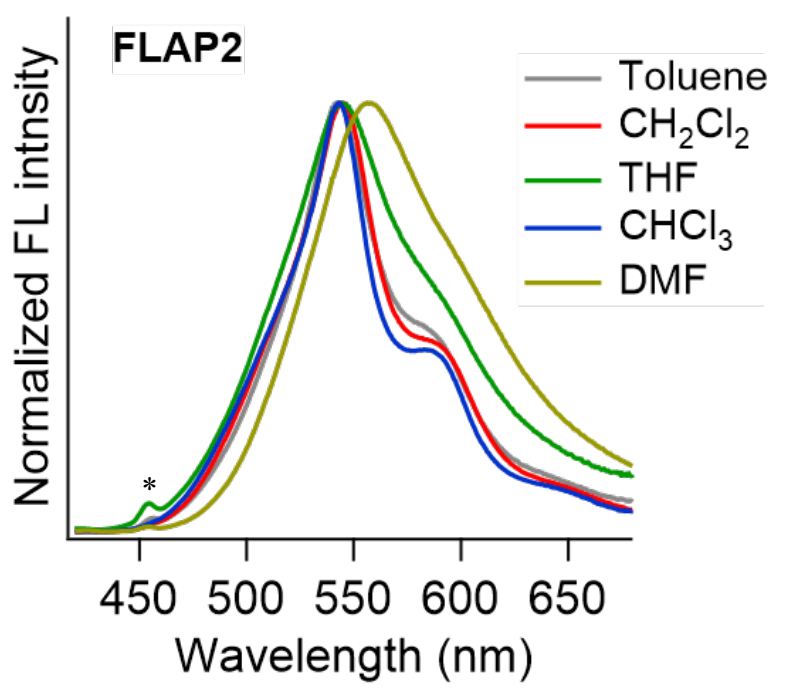

Figure S4.3. Fluorescence spectra of FLAP2 in various solvents with the concentration of $8 \times 10^{-6} \mathrm{M}$ (excitation wavelength $\lambda_{\mathrm{ex}}=340 \mathrm{~nm}$ ). Peaks marked with asterisk $\left(^{*}\right)$ indicate Raman peaks delivered from the solvent. 


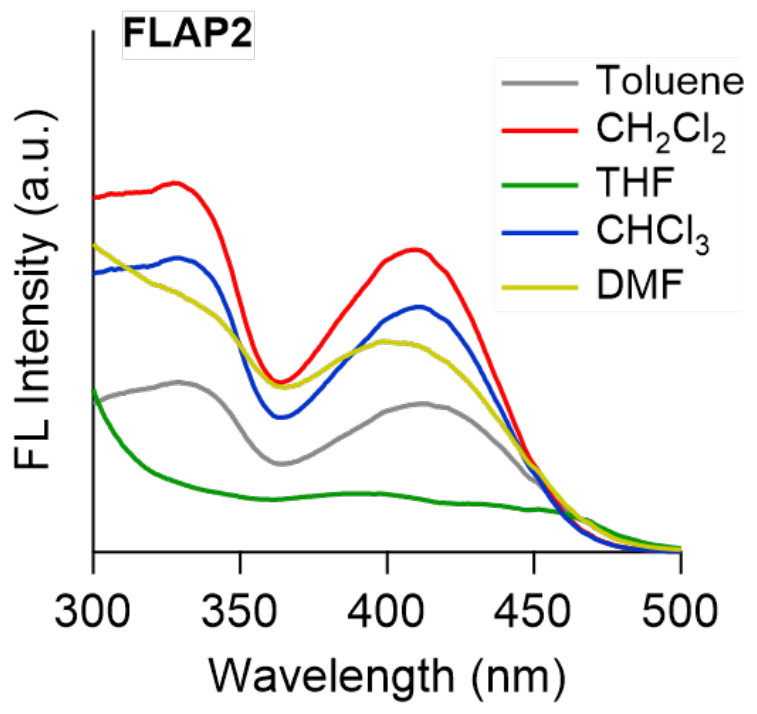

Figure S4.4. Excitation spectra of FLAP2 in various solvents with the concentration of $8 \times 10^{-6}$ M (emission wavelengths $\left.\lambda_{\mathrm{em}}=545 \mathrm{~nm}\right)$.

Table S4.1. Absolute FL quantum yield $\Phi_{\mathrm{F}}$ of FLAP1 in various solvents (excitation wavelength $\lambda_{\mathrm{ex}}=340 \mathrm{~nm}$ ).

\begin{tabular}{c|c}
\hline & $\Phi_{\mathrm{F}}$ \\
\hline Toluene & $<0.001$ \\
\hline $\mathrm{CH}_{2} \mathrm{Cl}_{2}$ & $<0.001$ \\
\hline $\mathrm{THF}$ & $<0.001$ \\
\hline $\mathrm{CHCl}_{3}$ & $<0.001$ \\
\hline $\mathrm{DMF}$ & $<0.001$ \\
\hline
\end{tabular}

Table S4.2. Absolute FL quantum yield $\Phi_{\mathrm{F}}$ and FL lifetime $\tau_{\mathrm{F}}$ of FLAP2 in various solvents or a PMMA matrix

\begin{tabular}{|c|c|c|c|}
\hline & $\Phi_{\mathrm{F}}$ & $\tau_{\mathrm{F}}$ & $\chi^{2}$ \\
\hline \multirow{2}{*}{ Toluene } & \multirow{2}{*}{0.015} & $490 \mathrm{ps}$ & $4.10\left(1^{\text {st }}\right.$ order fitting $)$ \\
\hline & & 480 ps $(>99 \%), 2.5$ ns $(0.4 \%)$ & $1.10\left(2^{\text {nd }}\right.$ order fitting $)$ \\
\hline $\mathrm{CH}_{2} \mathrm{Cl}_{2}$ & 0.028 & $480 \mathrm{ps}$ & $1.31\left(1^{\text {st }}\right.$ order fitting $)$ \\
\hline \multirow{2}{*}{ THF } & \multirow{2}{*}{0.017} & 480 ps & $6.48\left(1^{\text {st }}\right.$ order fitting $)$ \\
\hline & & $430 \mathrm{ps}(>99 \%), 1.9 \mathrm{~ns}(0.8 \%)$ & $1.18\left(2^{\text {nd }}\right.$ order fitting $)$ \\
\hline $\mathrm{CHCl}_{3}$ & 0.021 & $500 \mathrm{ps}$ & $1.31\left(1^{\text {st }}\right.$ order fitting $)$ \\
\hline $\mathrm{DMF}$ & 0.061 & 890 ps & $1.04\left(1^{\text {st }}\right.$ order fitting $)$ \\
\hline \multirow{3}{*}{ PMMA } & \multirow{3}{*}{0.012} & $1.1 \mathrm{~ns}$ & $76.4\left(1^{\text {st }}\right.$ order fitting $)$ \\
\hline & & 530 ps $(92 \%), 3.0$ ns $(8 \%)$ & $6.32\left(2^{\text {nd }}\right.$ order fitting $)$ \\
\hline & & $290 \mathrm{~ns}(80 \%), 1.3 \mathrm{~ns}(19 \%), 5.3 \mathrm{~ns}(2 \%)$ & $1.32\left(3^{\text {rd }}\right.$ order fitting $)$ \\
\hline
\end{tabular}

Excitation wavelength $\lambda_{\mathrm{ex}}=400 \mathrm{~nm}$ for $\Phi_{\mathrm{F}}$, and $405 \mathrm{~nm}$ for $\tau_{\mathrm{F}}$. Emission wavelength $\lambda_{\mathrm{em}}=515 \mathrm{~nm}(\mathrm{PMMA})$ and $545 \mathrm{~nm}$ (other solvents) for $\tau_{\mathrm{F}}$. The PMMA matrix was prepared by the following method: FLAP1 $(0.1 \mathrm{wt} \%)$ and PMMA (typical $M_{\mathrm{w}}$ $=15000$, Aldrich) were dissolved in toluene, and this solution was dropped onto a quartz plate and dried under vacuum for $12 \mathrm{~h}$. 


\subsection{Photostability under UV irradiation}
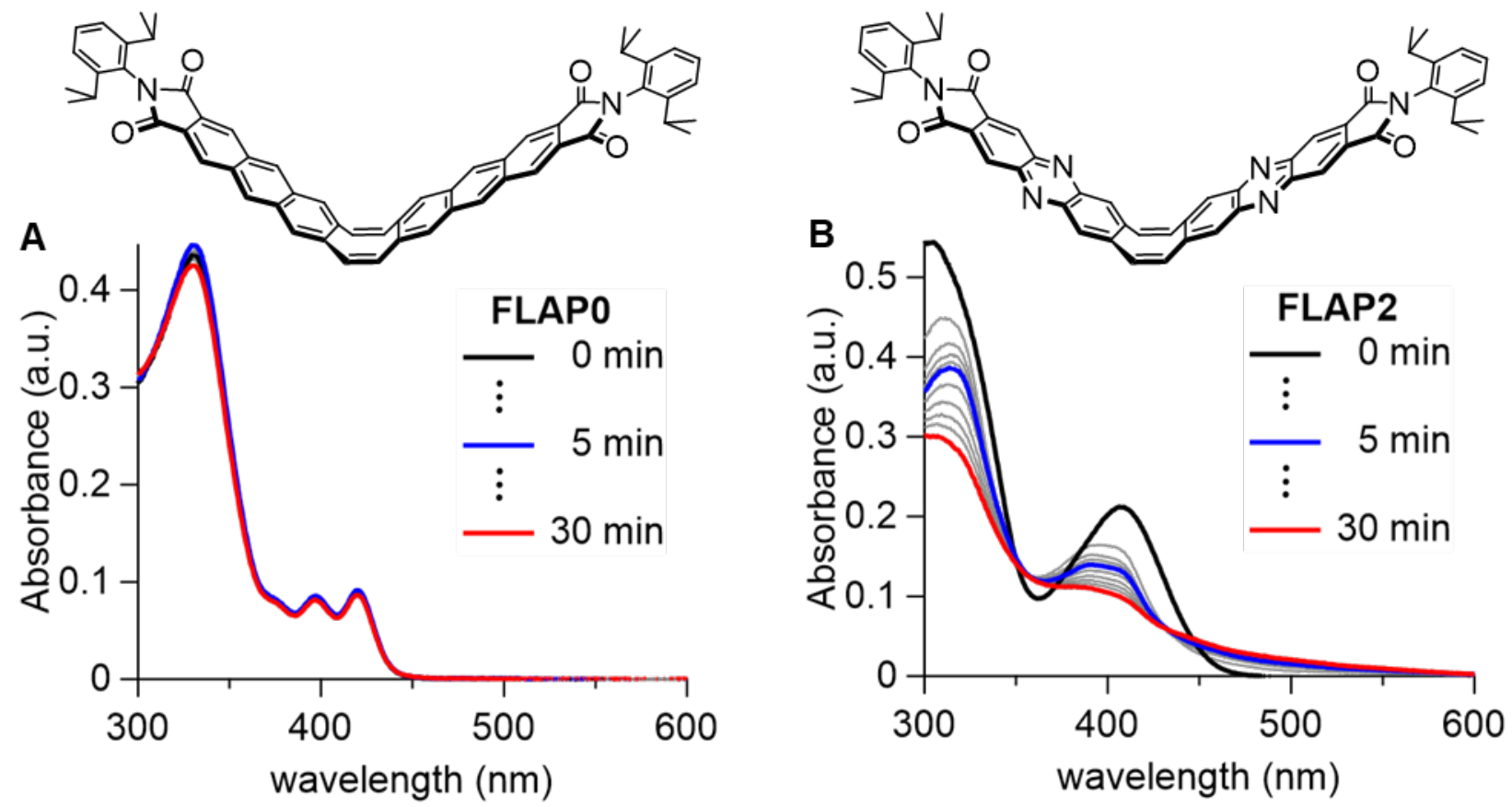

Figure S4.5. Evaluation of photostability of (A) FLAP0 and (B) FLAP2. Concentration of FLAP0 and FLAP2 in CHCl FH $_{3}$ was adjusted with the absorbance of 0.1 at $365 \mathrm{~nm}$. The $\mathrm{CHCl}_{3}$ solutions were stirred under continuous spot irradiation using a UV-LED light source $\left(\lambda=365 \mathrm{~nm}, 100 \mathrm{~mW} \mathrm{~cm}^{-2}\right)$. Photodegradation was monitored by absorption spectroscopy of the solutions. 


\subsection{Fluorescence spectra in the mixed solvent system of DMSO/glycerol}
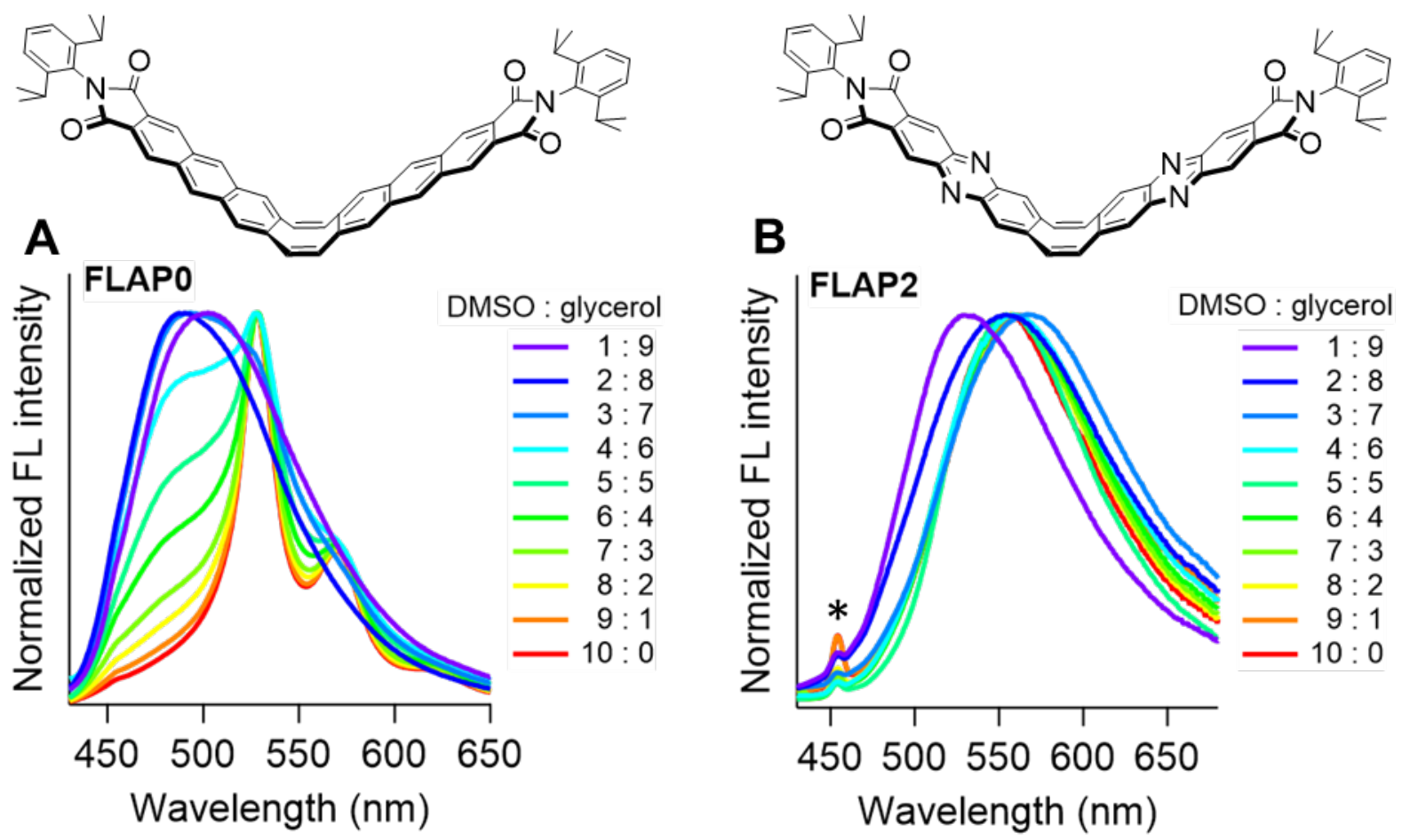

Figure S4.6. Viscosity-dependent fluorescence spectra of (A) FLAP0 and (B) FLAP2 in the mixed solvent system of DMSO/glycerol. The viscosity values of the mixed system were referenced from the literature ${ }^{[\mathrm{S} 7]}$, in which 668 (1:9), 338 (2:8), 169 (3:7), 86.4 (4:6), 44.9 (5:5), 23.7 (6:4), 12.8 (7:3), 7.0 (8:2), 3.9 (9:1), and $2.2 \mathrm{cP}$ (10:0), respectively. Excitation wavelength $\lambda_{\mathrm{ex}}=340 \mathrm{~nm}$. Peaks marked with asterisk (*) indicate Raman peaks delivered from the solvent. 


\subsection{Temperature dependence}
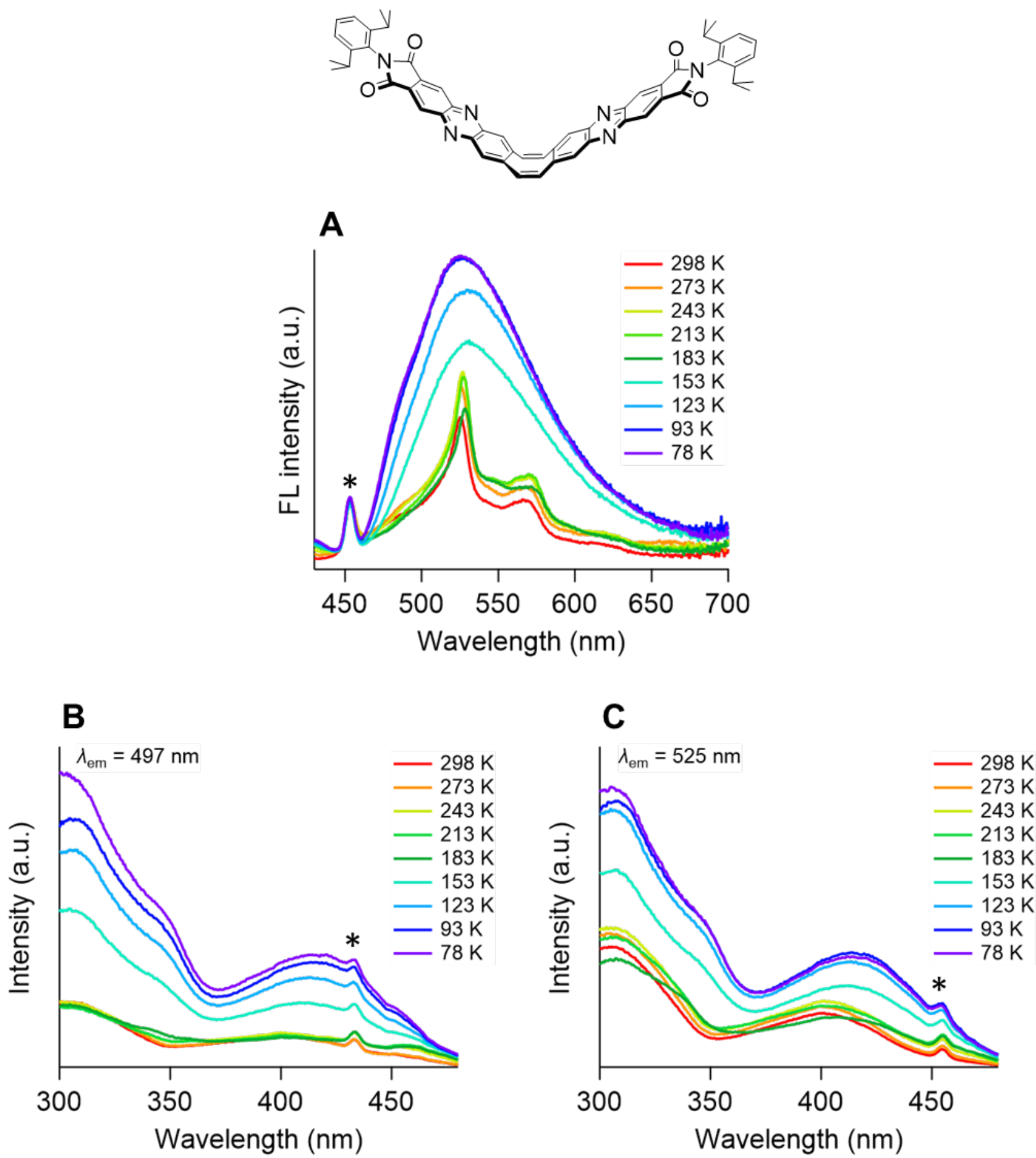

Figure S4.7. (A) Temperature dependent fluorescence spectra of FLAP2 in 3-methylpentane (melting point: $-118^{\circ} \mathrm{C}$ ).

Excitation wavelength $\lambda_{\mathrm{ex}}=400 \mathrm{~nm}$ for all measurements. (B, C) Temperature dependent excitation spectra of FLAP2 in 3methylpentane. Emission wavelengths: (B) $497 \mathrm{~nm}$ and (C) $525 \mathrm{~nm}$. Peaks marked with asterisk (*) indicate Raman peaks delivered from the solvent. 


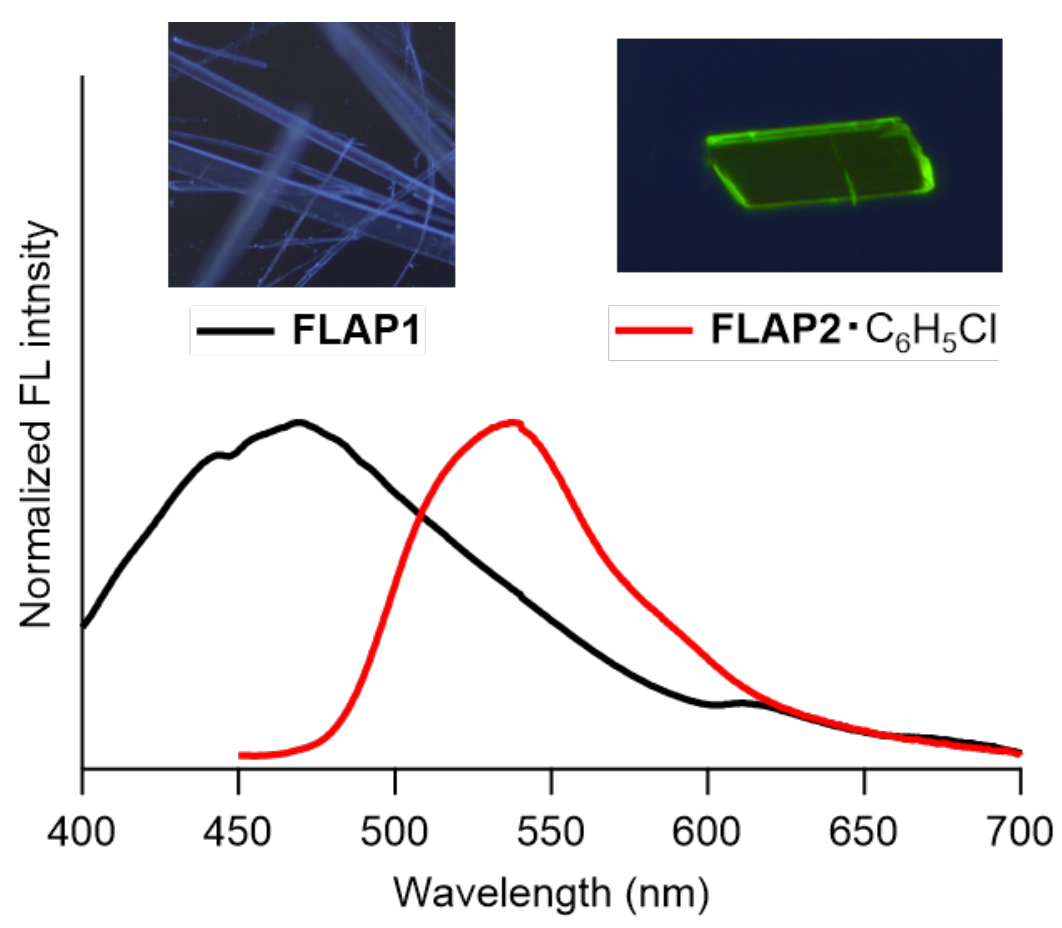

Figure S4.8. Fluorescence spectra of the crystals FLAP1 and FLAP2 $\cdot \mathrm{C}_{6} \mathrm{H}_{5} \mathrm{Cl}$.

\subsection{Fluorescence in a PMMA matrix}

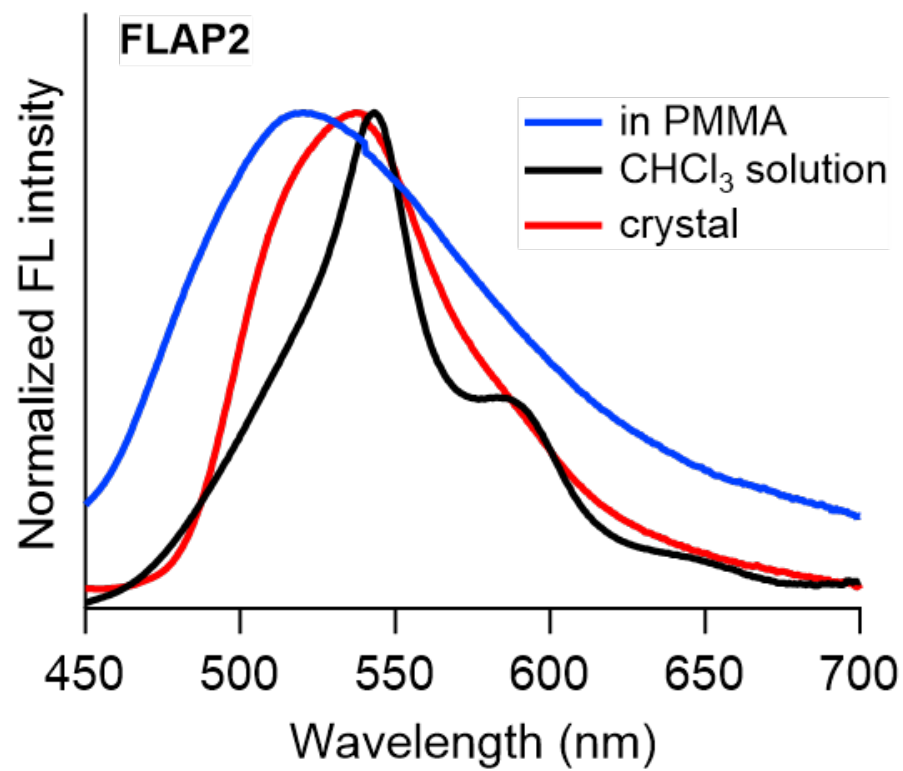

Figure S4.9. Fluorescence spectra of FLAP2 in a PMMA matrix, in a $\mathrm{CHCl}_{3}$ solution, and in the crystalline phase. The preparation method for the PMMA matrix is described in Table 6.2. 
A
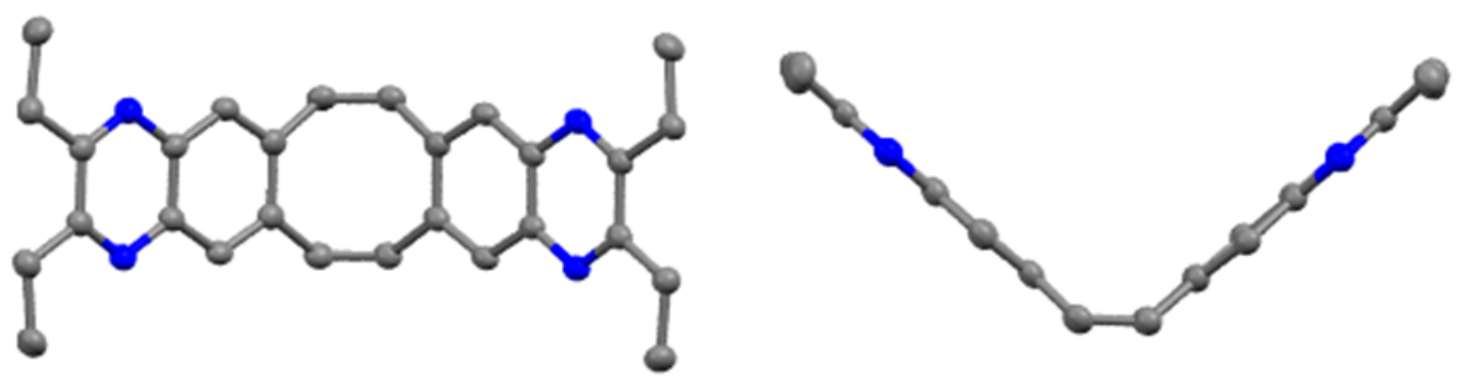

B
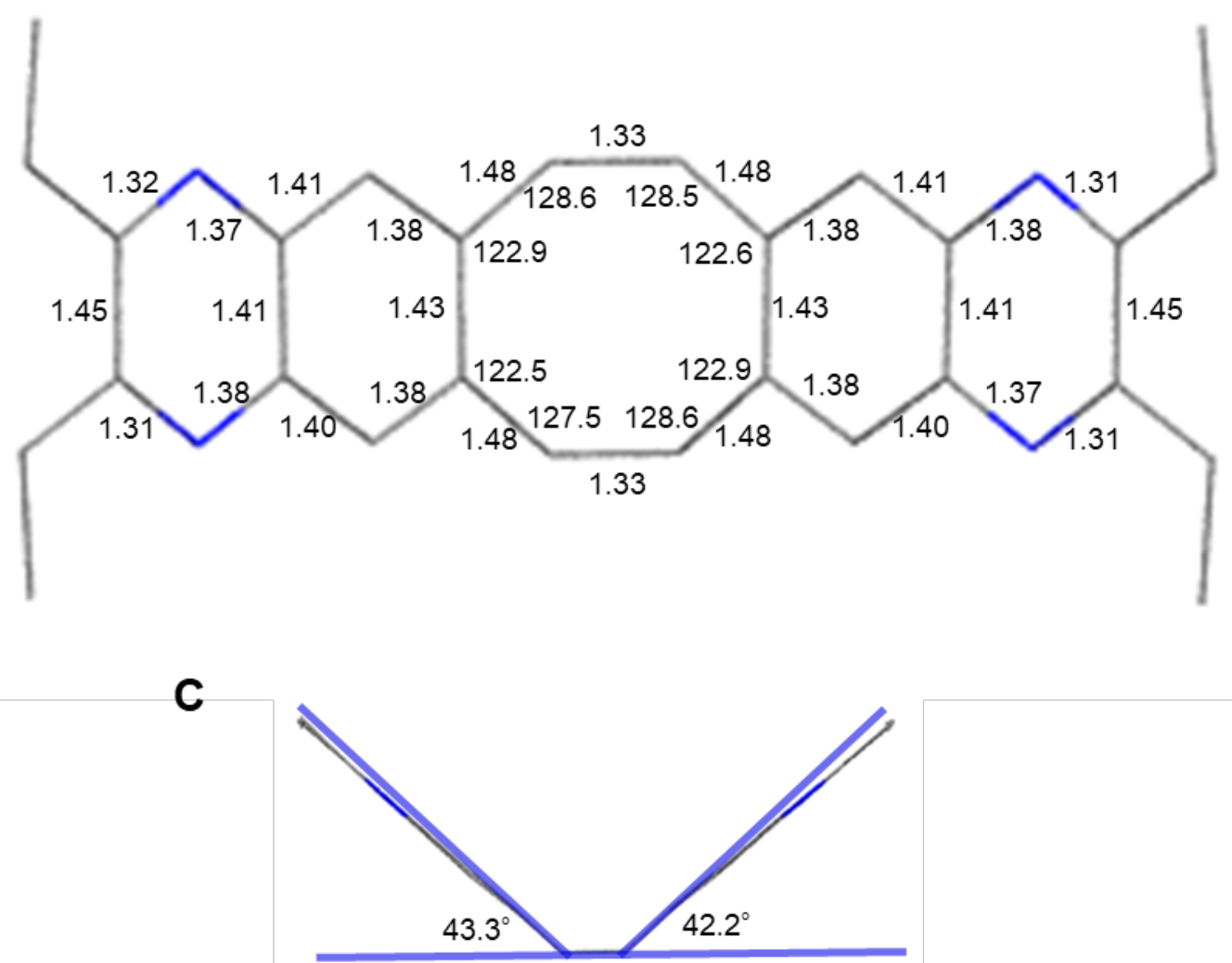

Figure S5.1. (A) Crystal structure of FLAP1 from different views. Thermal ellipsoids are scaled to $50 \%$ probability. Hydrogen atoms are omitted for clarity. (B) Representative bond lengths $(\AA)$ and bond angles $\left(^{\circ}\right)$ and (C) COT bending angles (see Figure S6.2 for the definition). 

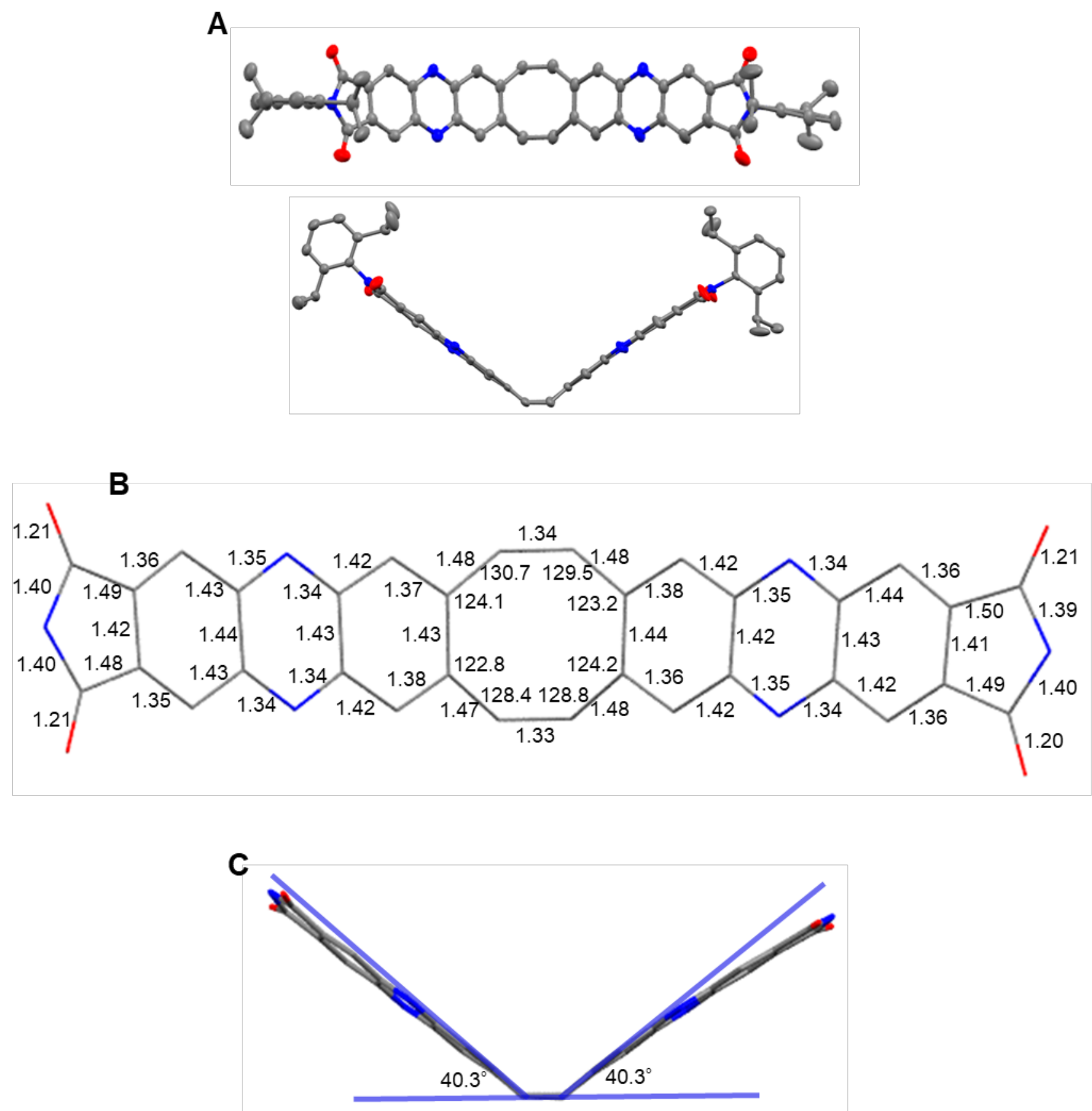

Figure S5.2. (A) Crystal structure of FLAP2 from different views. Thermal ellipsoids are scaled to $50 \%$ probability. Hydrogen atoms and solvent molecules are omitted for clarity. (B) Representative bond lengths $(\AA)$ and bond angles $\left(^{\circ}\right)$ and (C) COT bending angles (see Figure $\mathbf{S 6 . 2}$ for the definition). The 2,6-diisopropylphenyl groups are omitted for clarity. 
Table S5.1. Crystal Data of FLAP1 and FLAP2

\begin{tabular}{lll}
\hline & \multicolumn{1}{c}{ FLAP1 } & \multicolumn{1}{c}{ FLAP2 } \\
\hline Formula & $\mathrm{C}_{28} \mathrm{H}_{28} \mathrm{~N}_{4}$ & $\mathrm{C}_{56} \mathrm{H}_{46} \mathrm{~N}_{6} \mathrm{O}_{4} \cdot 2\left(\mathrm{C}_{6} \mathrm{H}_{5} \mathrm{Cl}\right)$ \\
FW & 420.54 & 1092.08 \\
Crystal system & triclinic & triclinic \\
Space Group & $P-1(\mathrm{No} .2)$ & $P-1(\mathrm{No} .2)$ \\
$a / \AA$ & $4.6738(1)$ & $8.7565(1)$ \\
$b / \AA$ & $15.5587(5)$ & $16.9417(3)$ \\
$c / \AA$ & $16.6456(5)$ & $20.1692(4)$ \\
$\alpha /{ }^{\circ}$ & $68.373(3)$ & $68.493(2)$ \\
$\beta /{ }^{\circ}$ & $88.012(2)$ & $82.020(1)$ \\
$\gamma /{ }^{\circ}$ & $81.681(2)$ & $81.345(1)$ \\
$V / \AA$ & $1113.16(6)$ & $2740.66(9)$ \\
$Z$ & 2 & 2 \\
$T / \mathrm{K}$ & 93 & 93 \\
$R_{1}[I>2 \sigma(I)]$ & 0.0494 & 0.0800 \\
$w R_{2}$ (all data) & 0.1432 & 0.2317 \\
$\mathrm{GOF}$ & 1.057 & 1.027 \\
$\mathrm{CCDC}$ No. & 2081297 & 2081298 \\
\hline
\end{tabular}




\section{Theoretical calculations}

Model structures FLAP0', FLAP1', and FLAP2' were calculated, which have hydrogen atoms in place of the ethyl groups and 2,6-diisopropylphenyl groups of the corresponding compounds (Figure S6.1). All calculations were performed at the (TD) PBE0/6-31+G(d) level of theory, using the Gaussian 16 program ${ }^{[\mathrm{S} 8]}$. All energy minimum structures had no imaginary frequency. The COT bending angle was defined as the dihedral angle between the $\mathrm{C} 1-\mathrm{C} 2-\mathrm{C} 3$ and $\mathrm{C} 2-\mathrm{C} 3-\mathrm{C} 4$ planes in Figure S6.2. The $x, y$, and $z$ axes were defined as Figure $\mathrm{S} 6.3$ when a molecule has $C_{2 \mathrm{v}}$ symmetry.

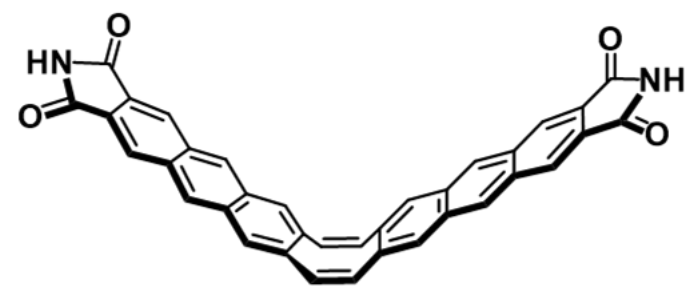

\section{FLAPO'}

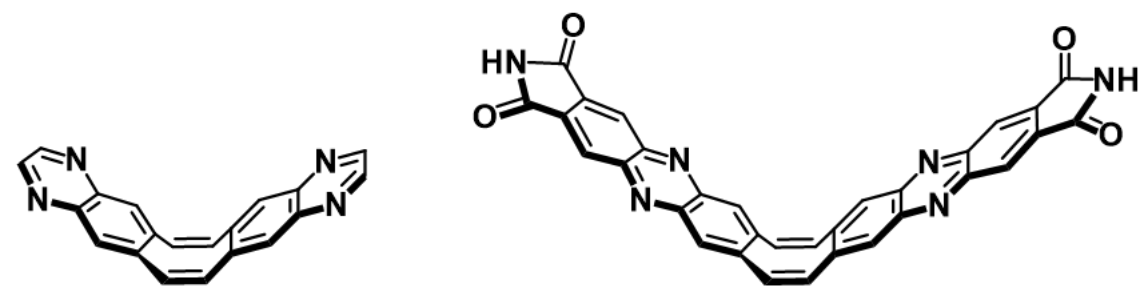

FLAP1'

FLAP2'

Figure S6.1. Structures of FLAP0', FLAP1', and FLAP2'.

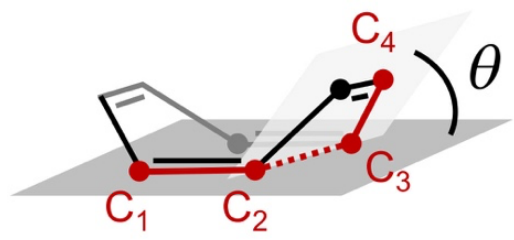

Figure S6.2. Definition of the COT bending angle.
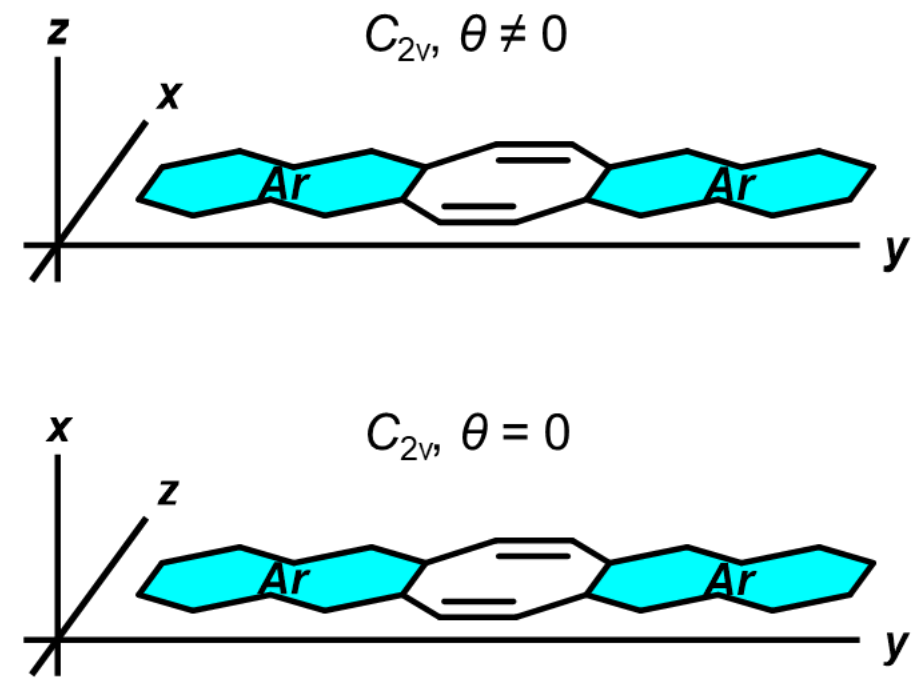

Figure S6.3. Definition of each axes. 

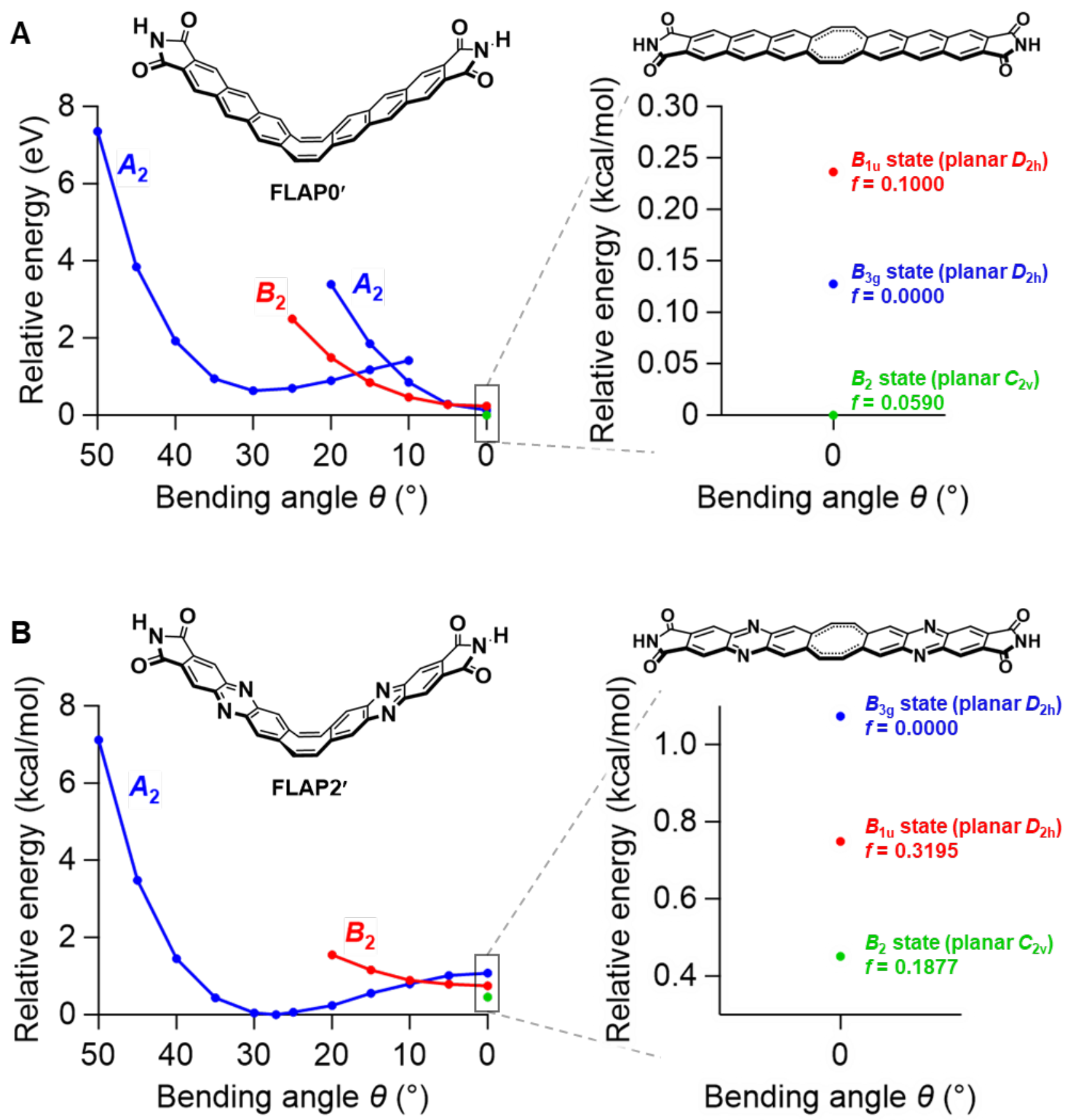

Figure S6.4. Energetically close excited states at $\theta=0$, calculated with different $\mathrm{S}_{1}$ optimized geometries (See the following pages).

(A) Three states of FLAP0' were obtained, as shown in Figure S6.6 $\left(B_{2}\right)$, S6.7 $\left(B_{1 \mathrm{u}}\right)$, and S6.8 $\left(B_{3 \mathrm{~g}}\right)$, respectively.

(B) Three states of FLAP2' were obtained, as shown in Figure S6.12 $\left(B_{2}\right)$, S6.13 $\left(B_{1 \mathrm{u}}\right)$, and S6.14 $\left(B_{3 \mathrm{~g}}\right)$, respectively. 

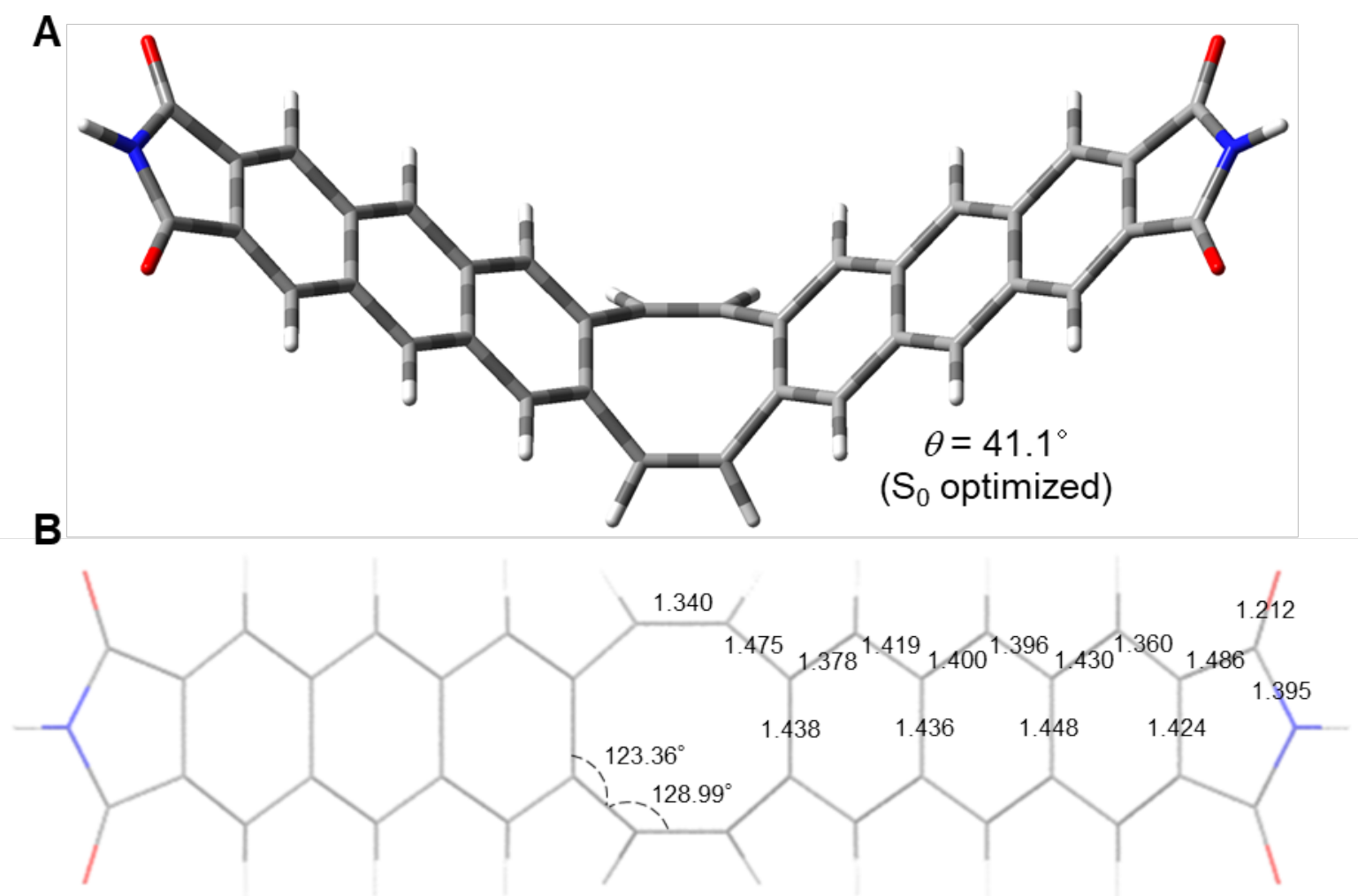

Figure S6.5. (A) $\mathrm{S}_{0}$ optimized geometry of FLAP0' with bent $C_{2 v}$ symmetry (See Figure S6.15). (B) Representative bond lengths $(\AA)$ and bond angles.
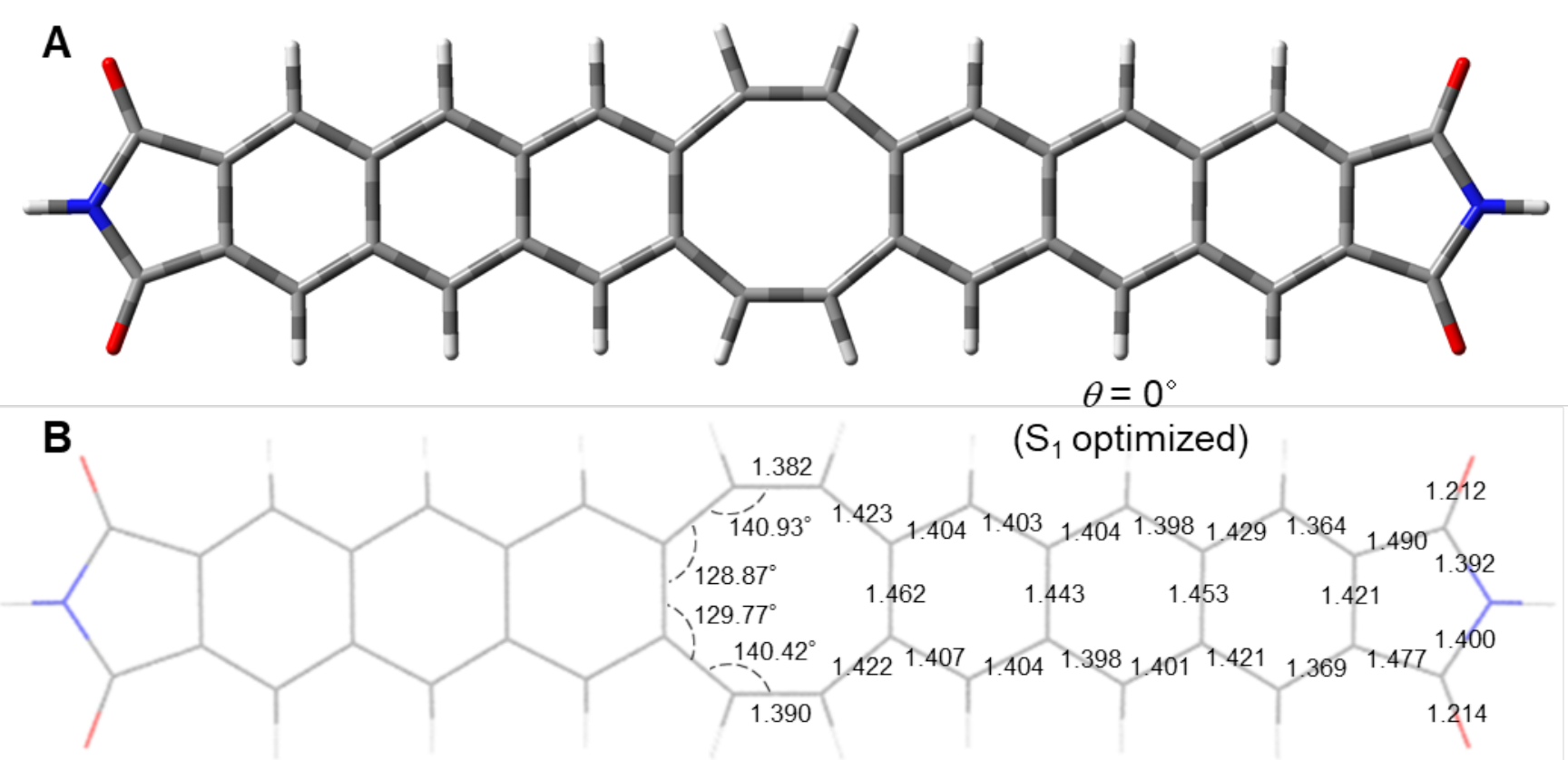

Figure S6.6. (A) $\mathrm{S}_{1}$ optimized geometry of FLAP0' ${ }^{\prime}$ with planar $C_{2 \mathrm{v}}$ symmetry that provides a $B_{2}$ state for the $\mathrm{S}_{1}$ configuration (See Figure S6.4 and S6.16A). (B) Representative bond lengths $(\AA)$ and bond angles. 
A

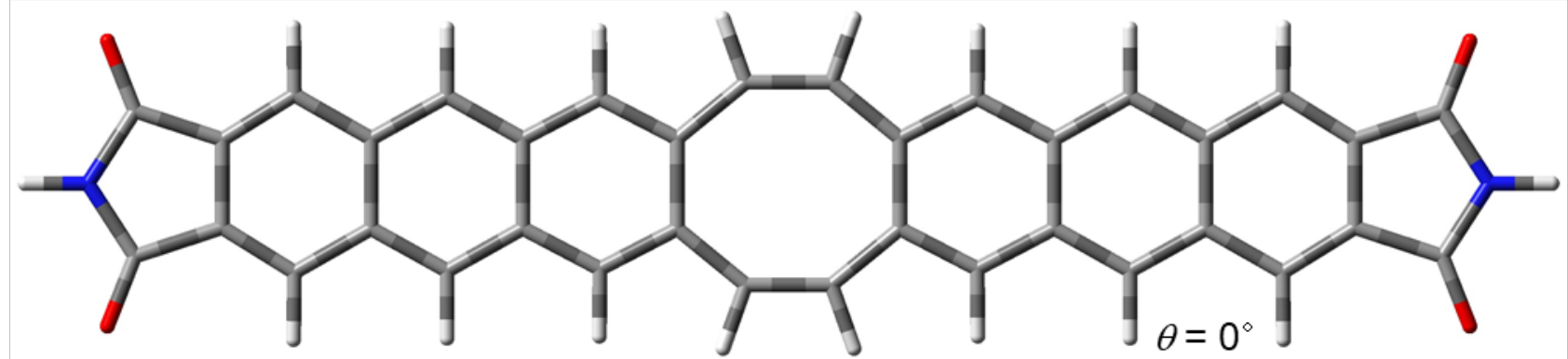

B

$\left(\mathrm{S}_{1}\right.$ optimized $)$

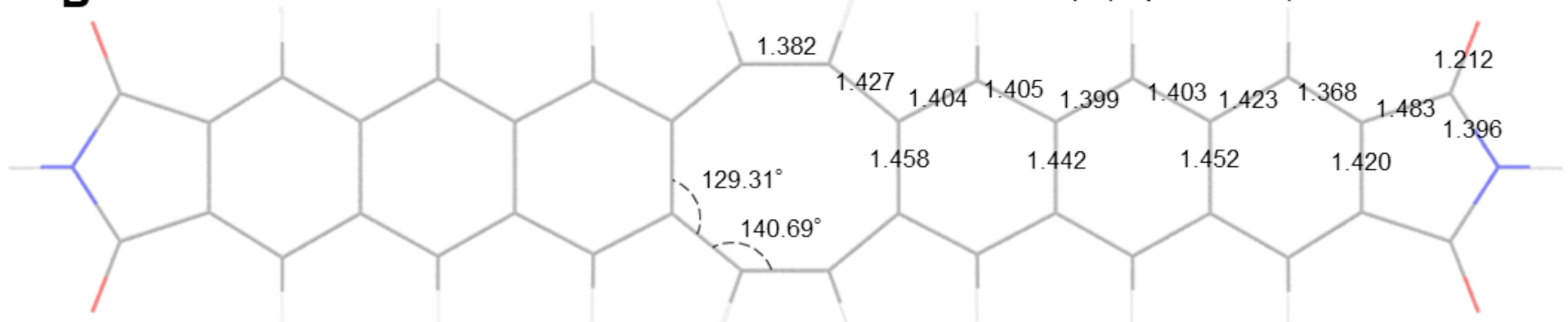

Figure S6.7. (A) $\mathrm{S}_{1}$ optimized geometry of FLAP0' with planar $D_{2 \mathrm{~h}}$ symmetry that provides a $B_{1 \mathrm{u}}$ state for the $\mathrm{S}_{1}$ configuration (See Figure S6.4 and S6.16B). (B) Representative bond lengths $(\AA)$ and bond angles.

A

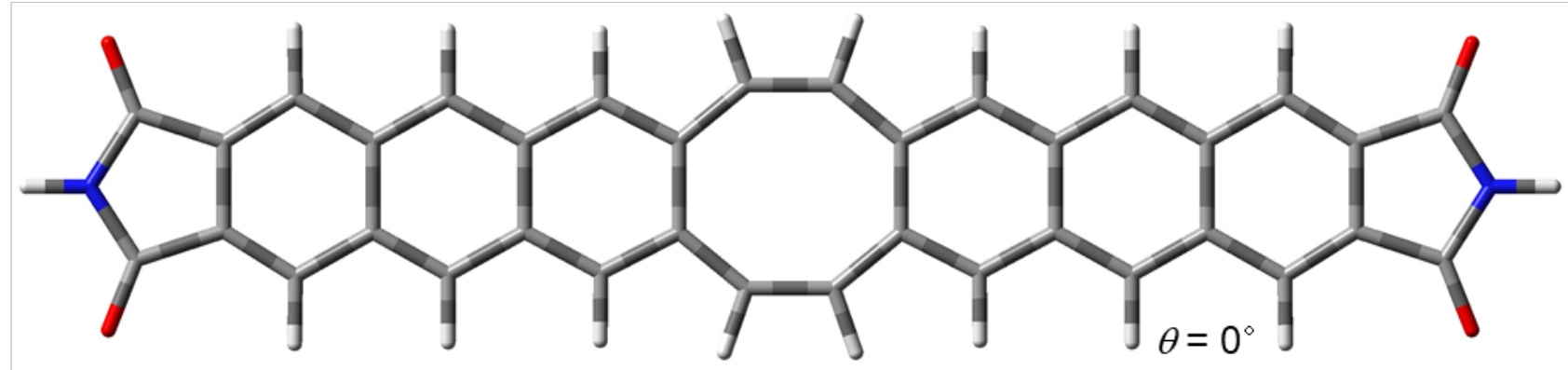

B

$\left(\mathrm{S}_{1}\right.$ optimized $)$

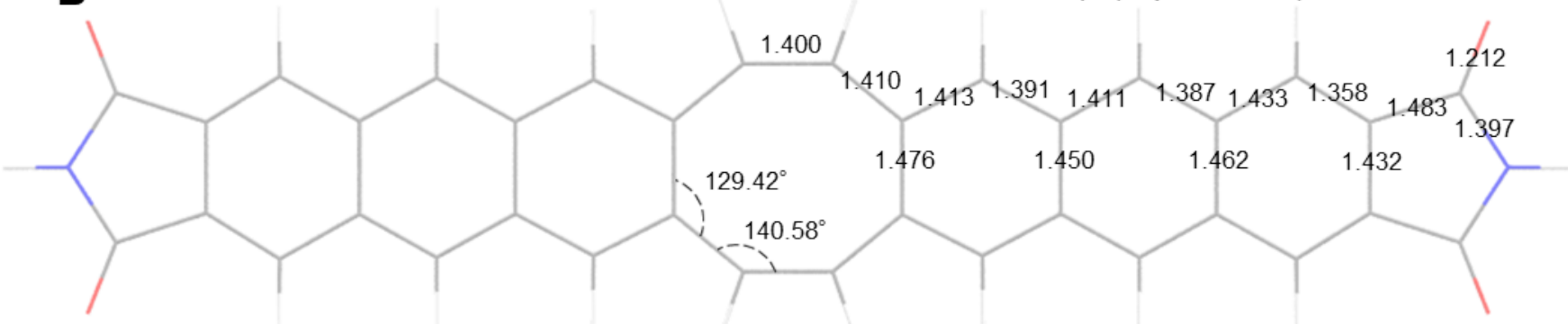

Figure S6.8. (A) $\mathrm{S}_{1}$ optimized geometry of FLAP0' with planar $D_{2 \mathrm{~h}}$ symmetry that provides a $B_{3 g}$ state for the $\mathrm{S}_{1}$ configuration (See Figure S6.4 and S6.16C). (B) Representative bond lengths ( $\AA$ ) and bond angles. 
A
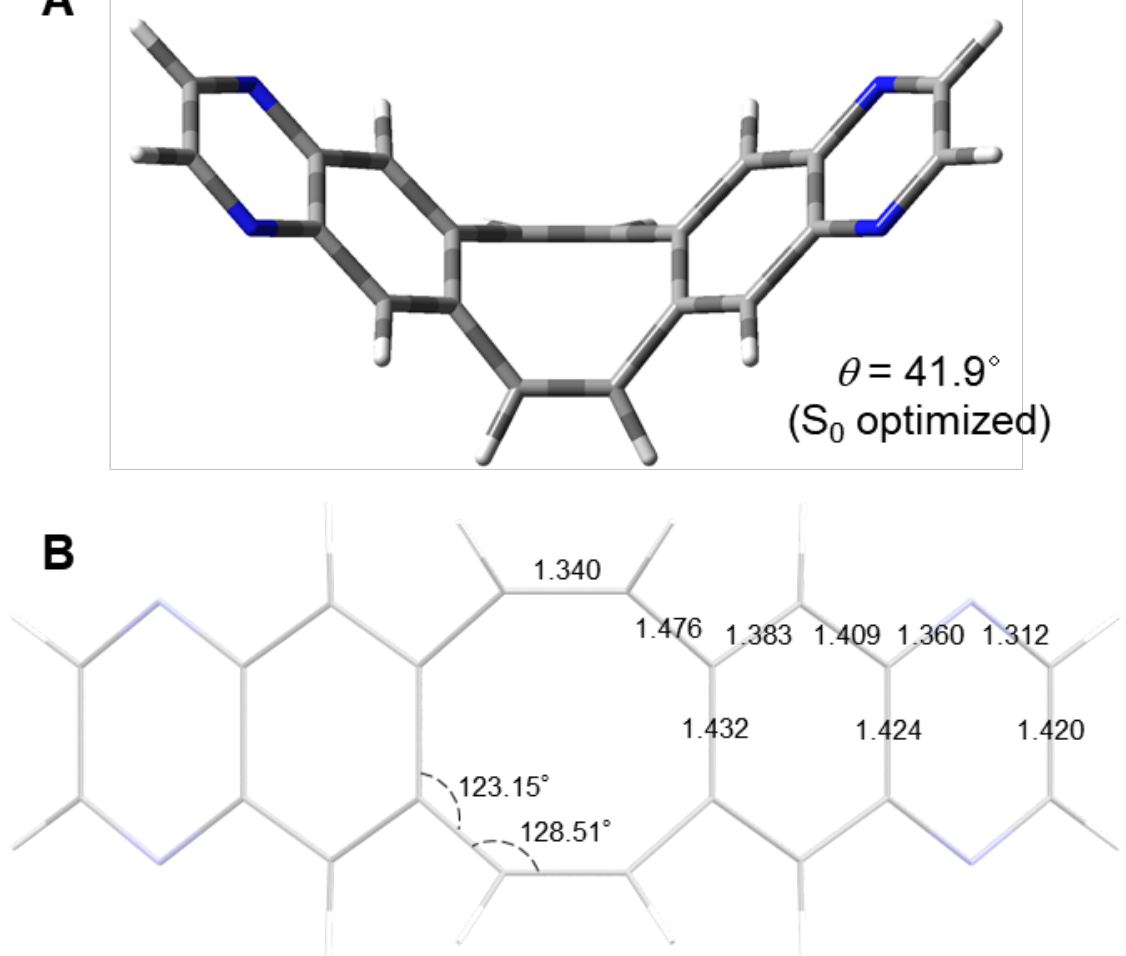

Figure S6.9. (A) $\mathrm{S}_{0}$ optimized geometry of FLAP1' with bent $C_{2 \mathrm{v}}$ symmetry (See Figure S6.17A). (B) Representative bond lengths $(\AA)$ and bond angles.

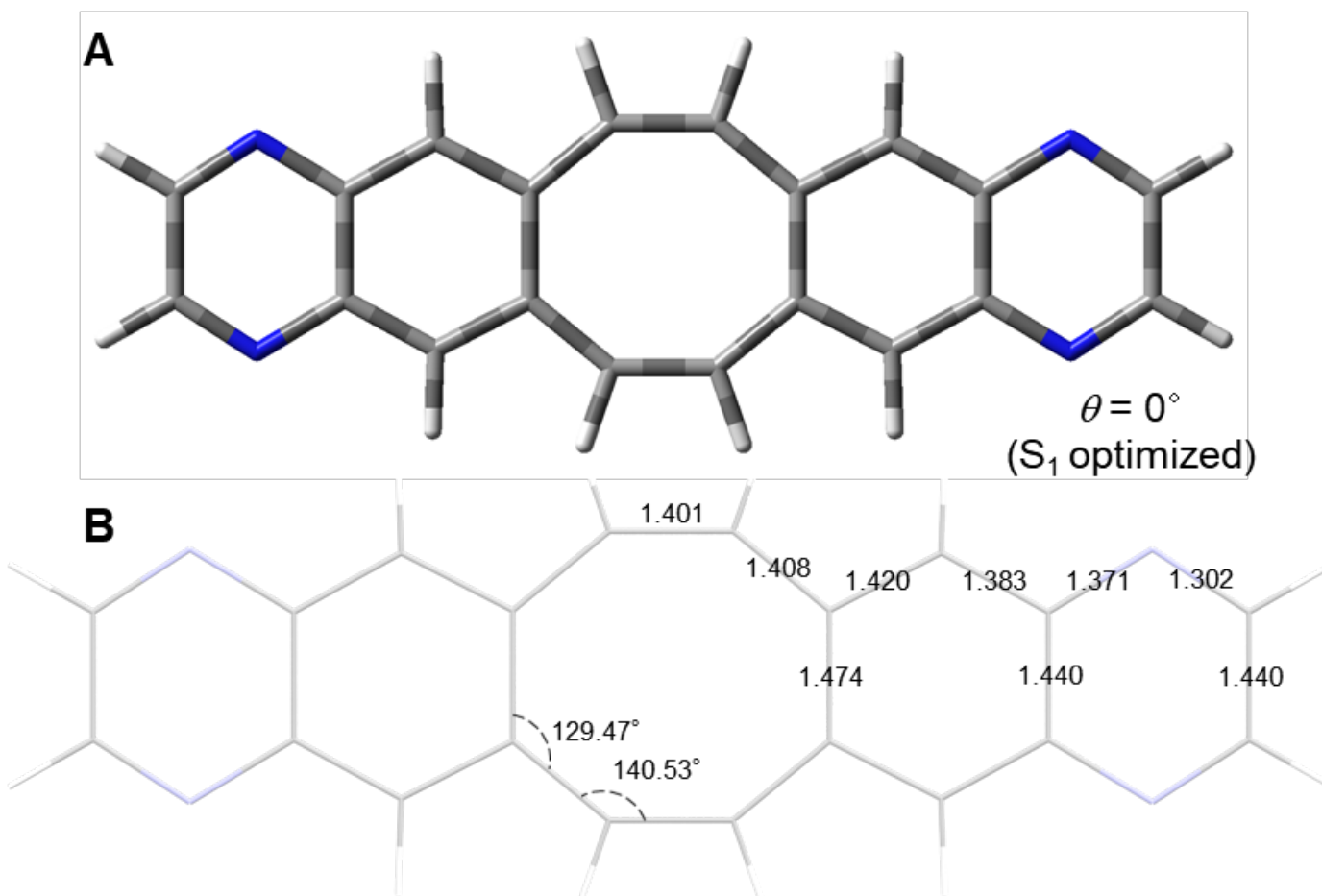

Figure S6.10. (A) $\mathrm{S}_{1}$ optimized geometry of FLAP1' with planar $D_{2 \mathrm{~h}}$ symmetry that provides a $B_{3 \mathrm{~g}}$ state for the $\mathrm{S}_{1}$ configuration (See Figure S6.17B). (B) Representative bond lengths $(\AA)$ and bond angles. 

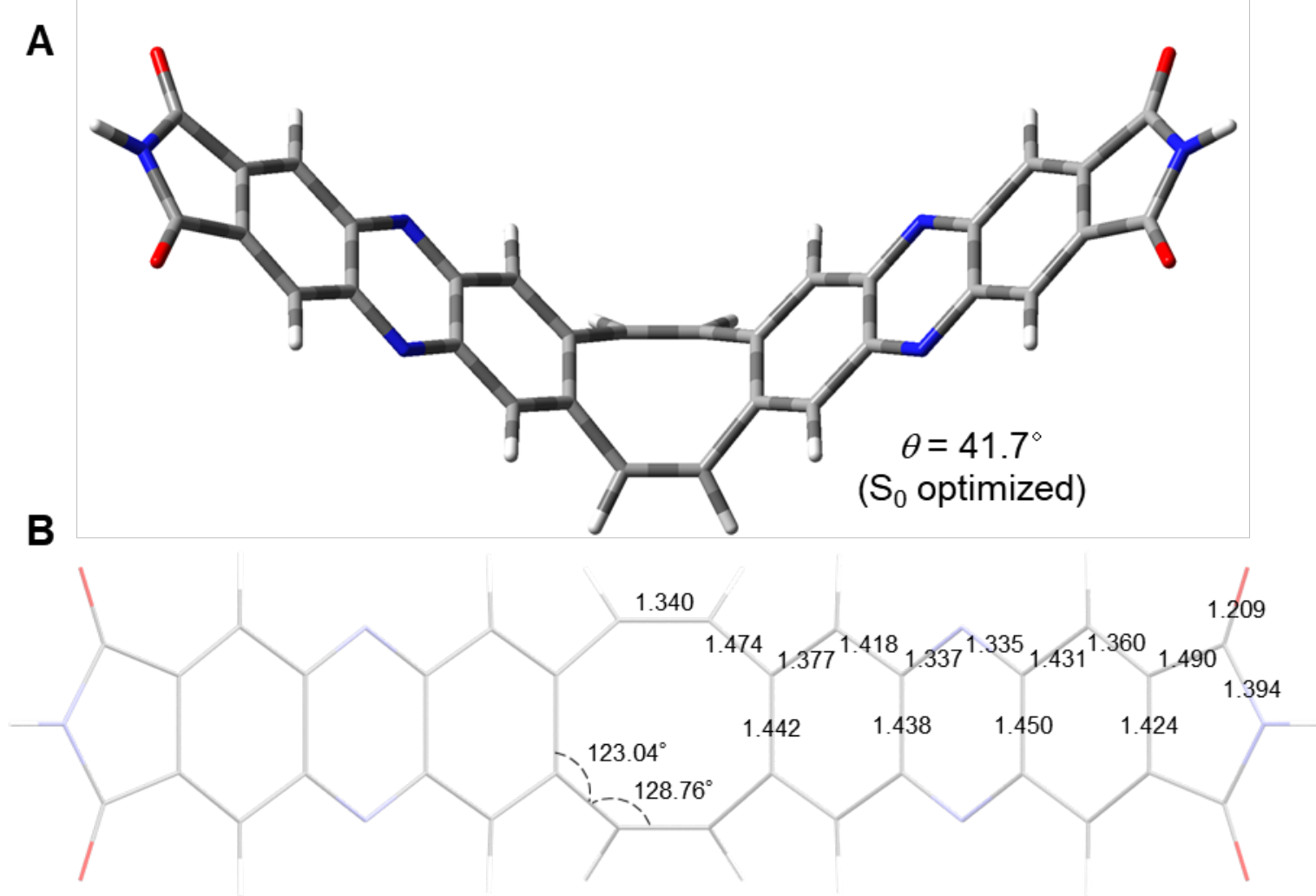

Figure S6.11. (A) $\mathrm{S}_{0}$ optimized geometry of FLAP2' with bent $C_{2 \mathrm{v}}$ symmetry (See Figure S6.18). (B) Representative bond lengths $(\AA)$ and bond angles.

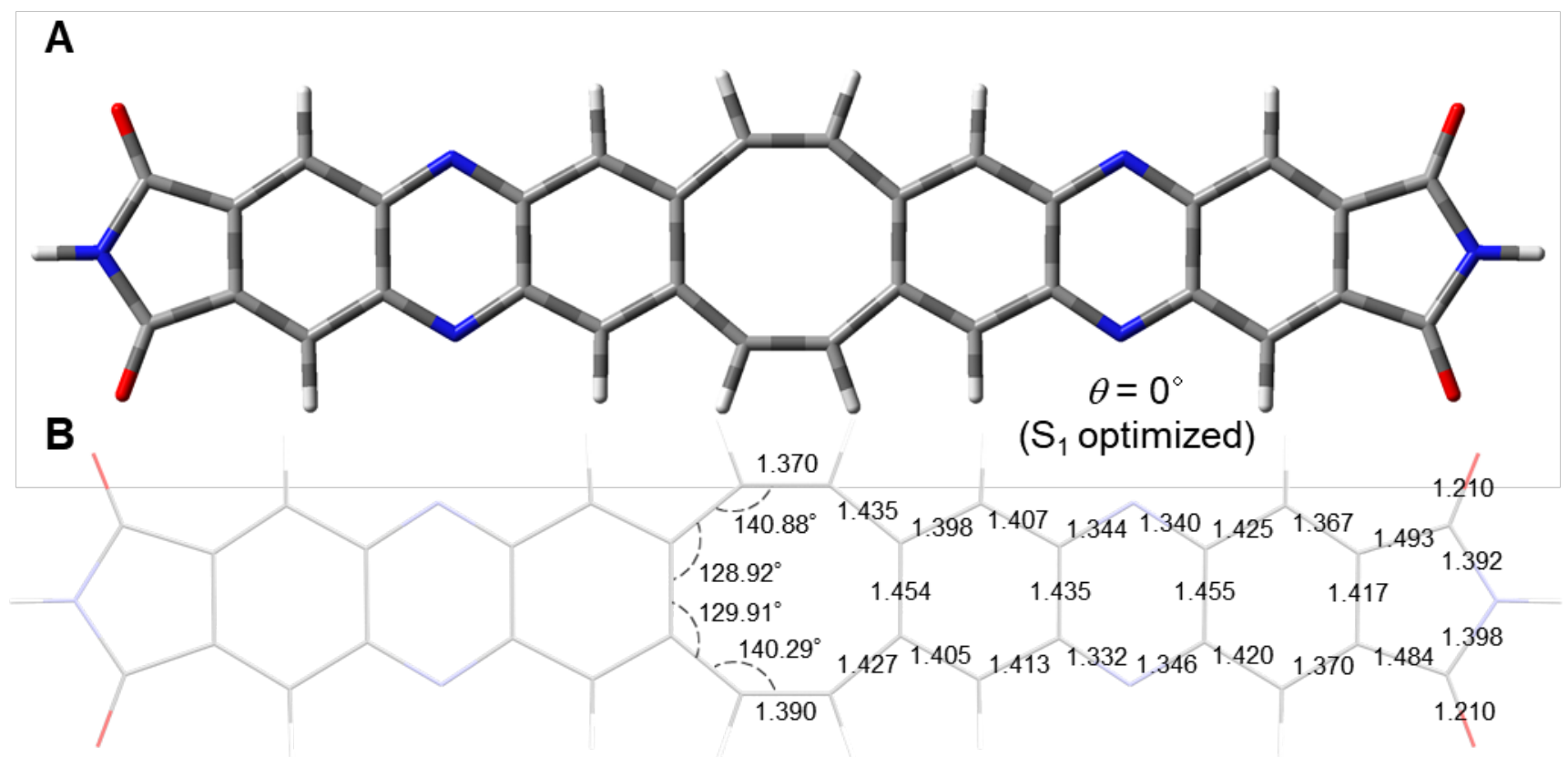

Figure S6.12. (A) $\mathrm{S}_{1}$ optimized geometry of FLAP2' with planar $C_{2 \mathrm{v}}$ symmetry that provides a $B_{2}$ state for the $\mathrm{S}_{1}$ configuration (See Figure S6.4 and S6.19A). (B) Representative bond lengths $(\AA)$ and bond angles. 
A
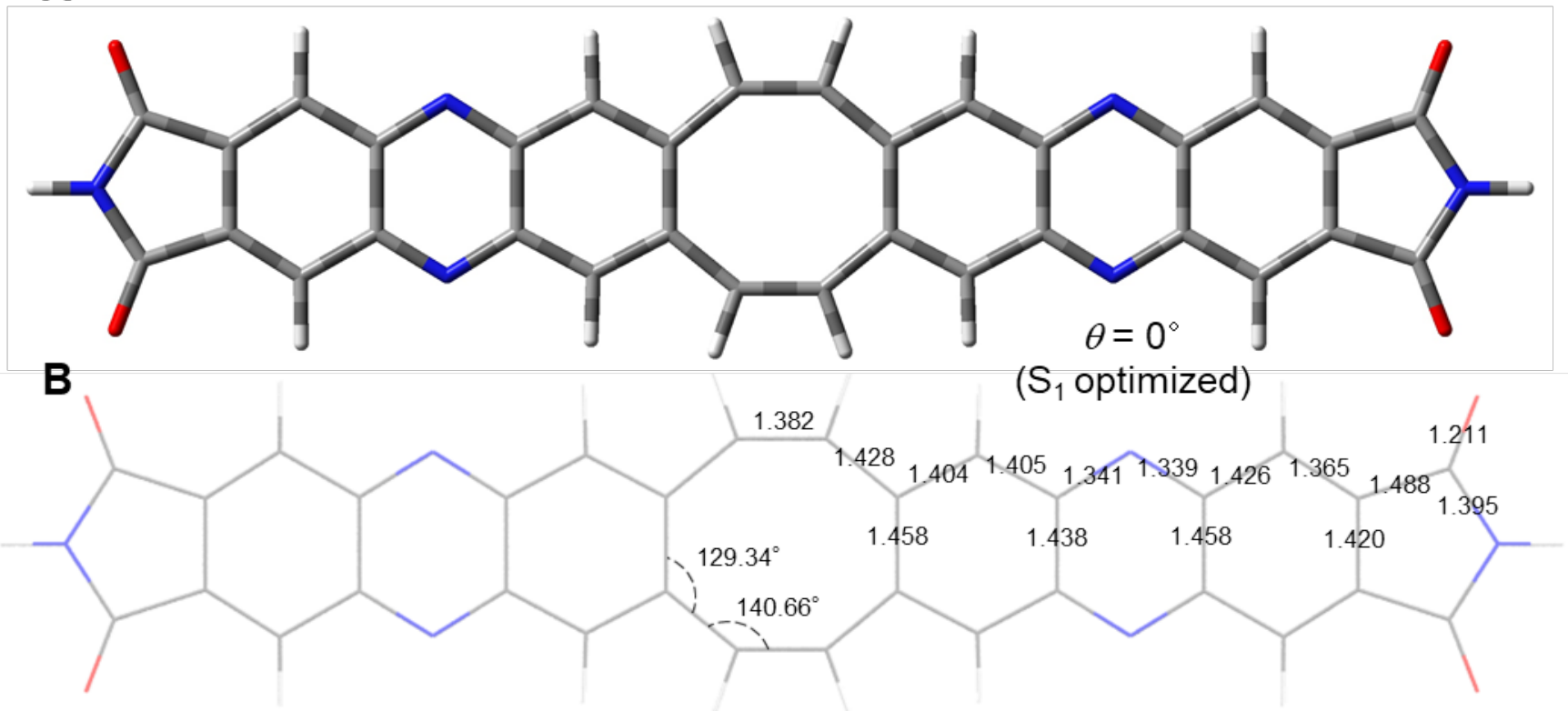

Figure S6.13. (A) $\mathrm{S}_{1}$ optimized geometry of FLAP2' with planar $D_{2 \mathrm{~h}}$ symmetry that provides a $B_{1 \mathrm{u}}$ state for the $\mathrm{S}_{1}$ configuration (See Figure S6.4 and S6.19B). (B) Representative bond lengths ( $\AA$ ) and bond angles.

A

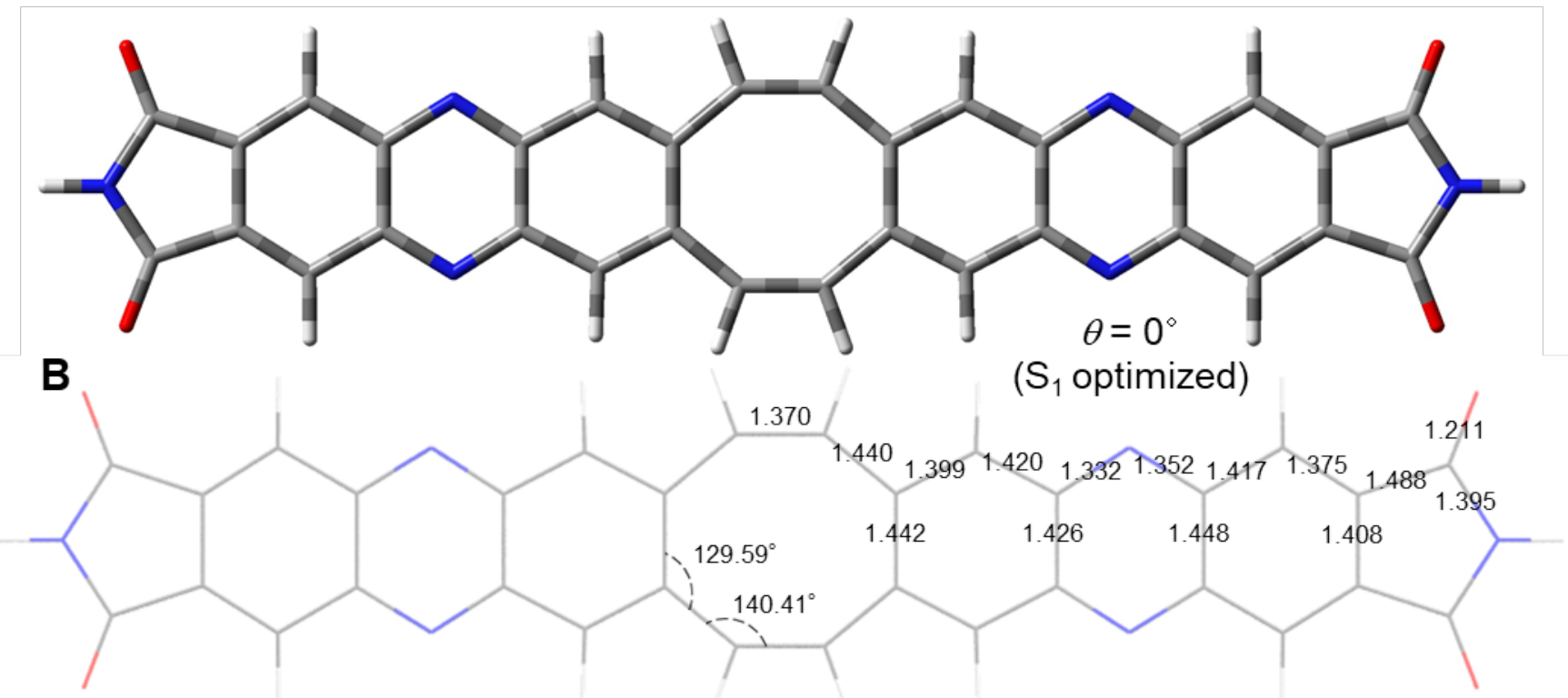

Figure S6.14. (A) $\mathrm{S}_{1}$ optimized geometry of FLAP2' with planar $D_{2 \mathrm{~h}}$ symmetry that provides a $B_{3 \mathrm{~g}}$ state for the $\mathrm{S}_{1}$ configuration (See Figure S6.4 and S6.19C). (B) Representative bond lengths $(\AA)$ and bond angles. 
6.3. Molecular orbitals of the $S_{0}$ and $S_{1}$ optimized geometries

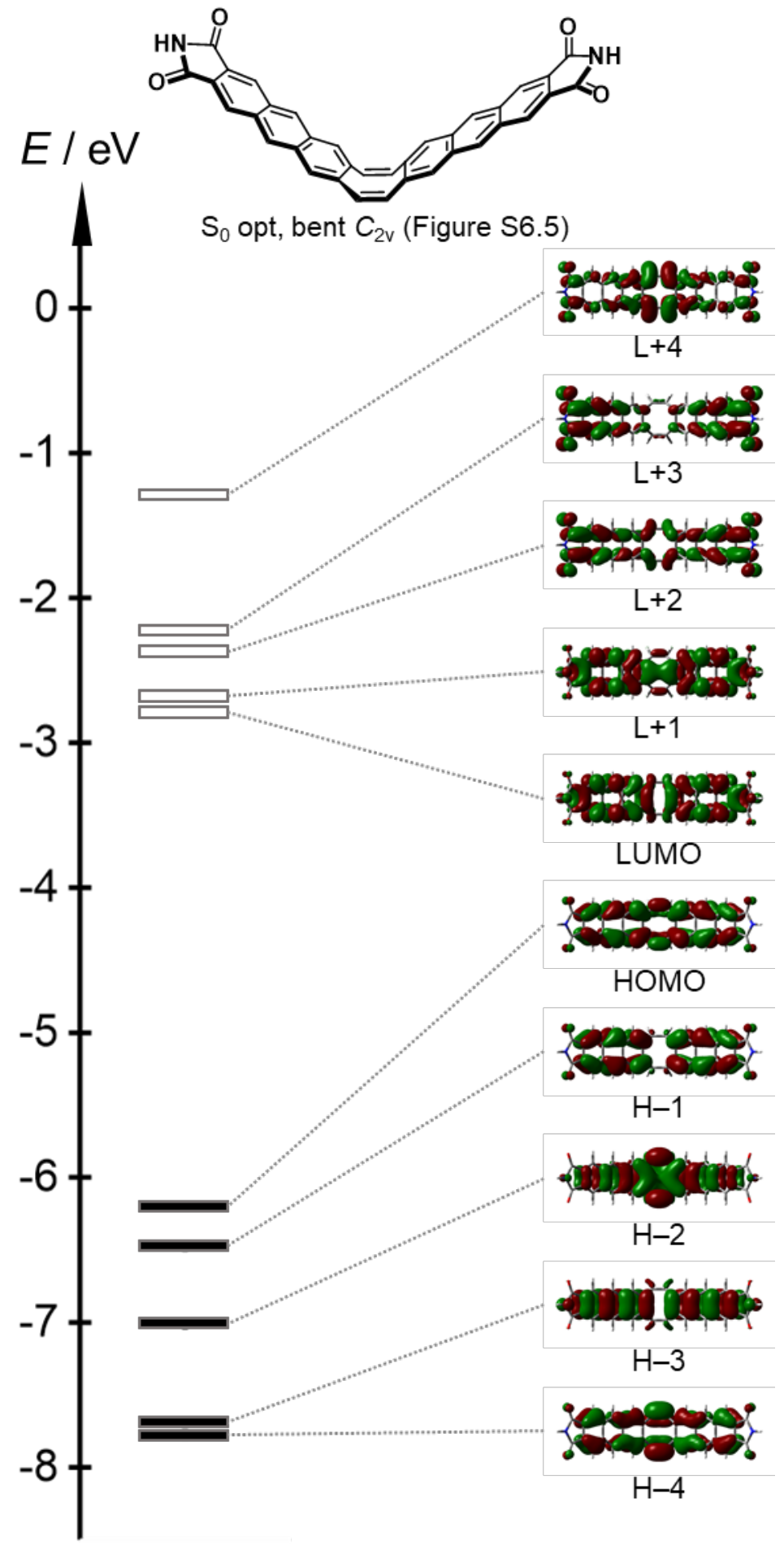

Figure S6.15. Kohn-Sham molecular orbitals and their energy levels of FLAP0' ${ }^{\prime}$ at the $\mathrm{S}_{0}$ geometry in Figure S6.5, optimized at the TD PBE0/6-31+G(d) level of theory.

Table S6.1. Excitation energy, configuration, and oscillator strength of the $\mathrm{S}_{0} \rightarrow \mathrm{S}_{\mathrm{n}}(\mathrm{n} \leq 3)$ transitions for the $\mathrm{S}_{0}$ optimized FLAP0' at the TD PBE0/6-31+G(d) level.

\begin{tabular}{|c|c|c|c|}
\hline Transition & Excitation energy & Configuration & Oscillator strength \\
\hline $\mathrm{S}_{0} \rightarrow \mathrm{S}_{1}\left(A_{2}\right)$ & $2.89 \mathrm{eV}(429 \mathrm{~nm})$ & $\mathrm{H} \rightarrow \mathrm{L}(96 \%)$ & 0.0000 \\
\hline $\mathrm{S}_{0} \rightarrow \mathrm{S}_{2}\left(B_{1}\right)$ & $2.99 \mathrm{eV}(414 \mathrm{~nm})$ & $\mathrm{H} \rightarrow \mathrm{L}+1(92 \%)$ & 0.0382 \\
\hline $\mathrm{S}_{0} \rightarrow \mathrm{S}_{3}\left(B_{2}\right)$ & $3.18 \mathrm{eV}(389 \mathrm{~nm})$ & $\begin{array}{l}\mathrm{H}-2 \rightarrow \mathrm{L}(16 \%) \\
\mathrm{H} \rightarrow \mathrm{L}+2(70 \%)\end{array}$ & 0.2441 \\
\hline
\end{tabular}




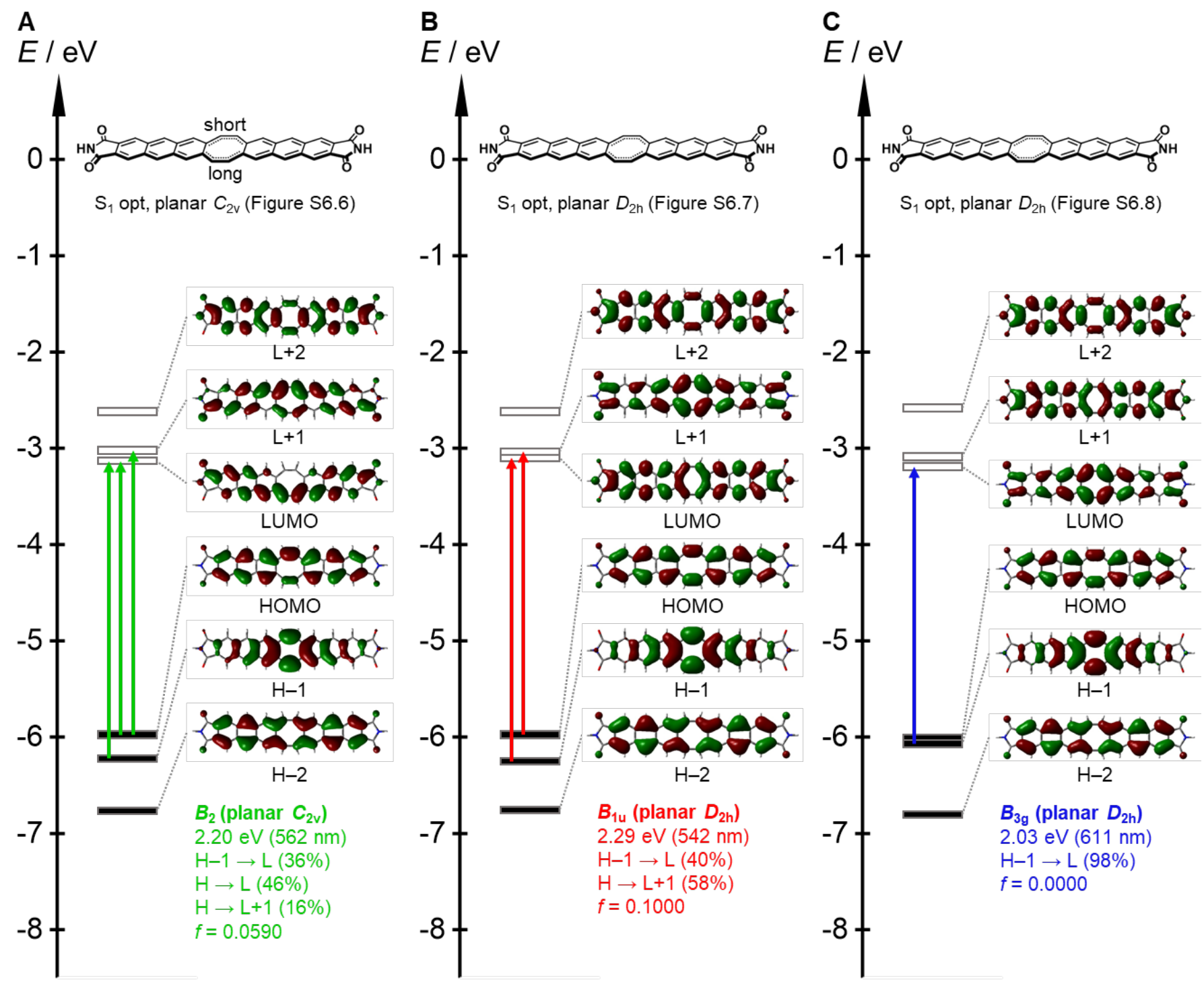

Figure S6.16. Kohn-Sham molecular orbitals and their energy levels of FLAP0' ${ }^{\prime}$ at the different $\mathrm{S}_{1}$ geometries in (A) Figure S6.6, (B) Figure S6.7, and (C) Figure S6.8, optimized at the TD PBE0/6-31+G(d) level of theory. 


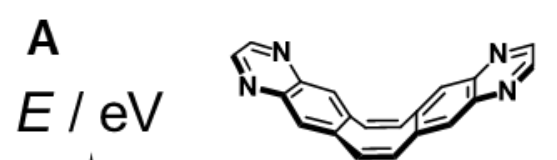

$\mathrm{S}_{0}$ opt, bent $\mathrm{C}_{2 \mathrm{v}}$ (Figure S6.9)

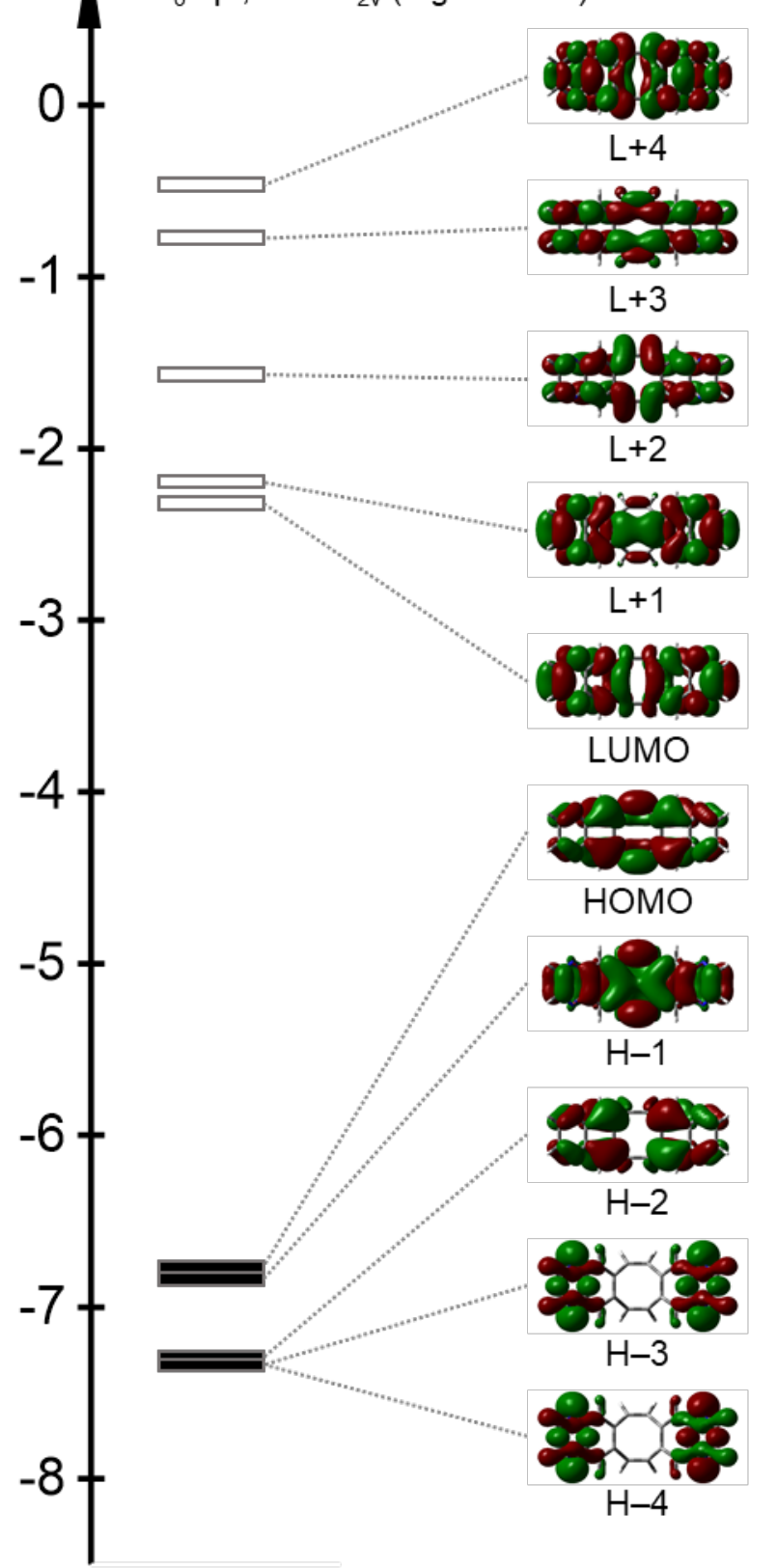

B

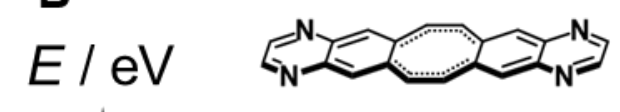

\ $\mathrm{S}_{1}$ opt, planar $D_{2 \mathrm{~h}}$ (Figure S6.10)

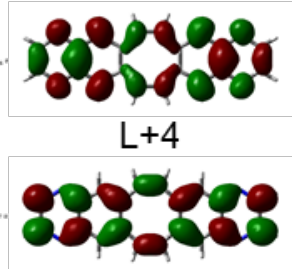

$L+3$

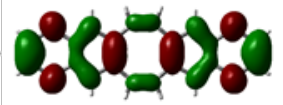

$L+2$

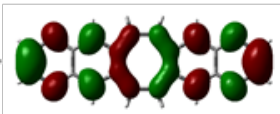

$\mathrm{L}+1$

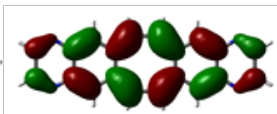

$B_{3 g}$ (planar $D_{2 h}$ )

LUMO

$-4 \int \quad \begin{aligned} & 1.83 \mathrm{eV}(676 \mathrm{~nm}) \\ & \mathrm{H} \rightarrow \mathrm{L}(100 \%) \\ & f=0.0000\end{aligned}$

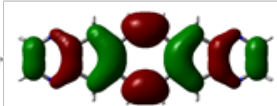

HOMO

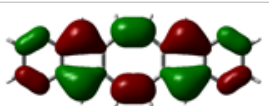

$\mathrm{H}-1$

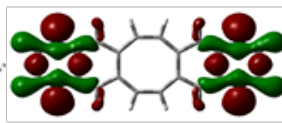

$\mathrm{H}-2$

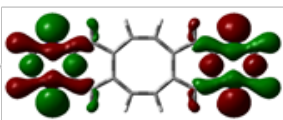

$\mathrm{H}-3$

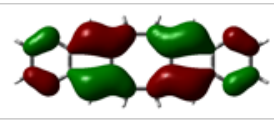

$\mathrm{H}-4$

Figure S6.17. Kohn-Sham molecular orbitals and their energy levels of FLAP1' at (A) the $\mathrm{S}_{0}$ geometry in Figure S6.9 and (B) at the $\mathrm{S}_{1}$ geometry in Figure $\mathrm{S} 6.10$, optimized at the (TD) PBE0/6-31+G(d) level.

Table S6.2. Excitation energy, configuration, and oscillator strength of the $S_{0} \rightarrow S_{n}(n \leq 4)$ transitions for the $S_{0}$ optimized FLAP1' at the TD PBE0/6-31+G(d) level.

\begin{tabular}{|c|c|c|c|}
\hline Transition & Excitation energy & Configuration & Oscillator strength \\
\hline $\mathrm{S}_{0} \rightarrow \mathrm{S}_{1}\left(B_{2}\right)$ & $3.54 \mathrm{eV}(350 \mathrm{~nm})$ & $\begin{array}{c}\mathrm{H}-4 \rightarrow \mathrm{L}+1(44 \%) \\
\mathrm{H}-3 \rightarrow \mathrm{L}(53 \%)\end{array}$ & 0.0079 \\
\hline $\mathrm{S}_{0} \rightarrow \mathrm{S}_{2}\left(A_{1}\right)$ & $3.55 \mathrm{eV}(350 \mathrm{~nm})$ & $\begin{array}{c}\mathrm{H}-4 \rightarrow \mathrm{L}(54 \%) \\
\mathrm{H}-3 \rightarrow \mathrm{L}+1(44 \%)\end{array}$ & 0.0016 \\
\hline $\mathrm{S}_{0} \rightarrow \mathrm{S}_{3}\left(A_{2}\right)$ & $3.76 \mathrm{eV}(330 \mathrm{~nm})$ & $\mathrm{H} \rightarrow \mathrm{L}(95 \%)$ & 0.0000 \\
\hline $\mathrm{S}_{0} \rightarrow \mathrm{S}_{4}\left(B_{2}\right)$ & $3.77 \mathrm{eV}(329 \mathrm{~nm})$ & $\begin{array}{c}\mathrm{H}-1 \rightarrow \mathrm{L}(86 \%) \\
\mathrm{H} \rightarrow \mathrm{L}+2(10 \%)\end{array}$ & 0.2182 \\
\hline
\end{tabular}




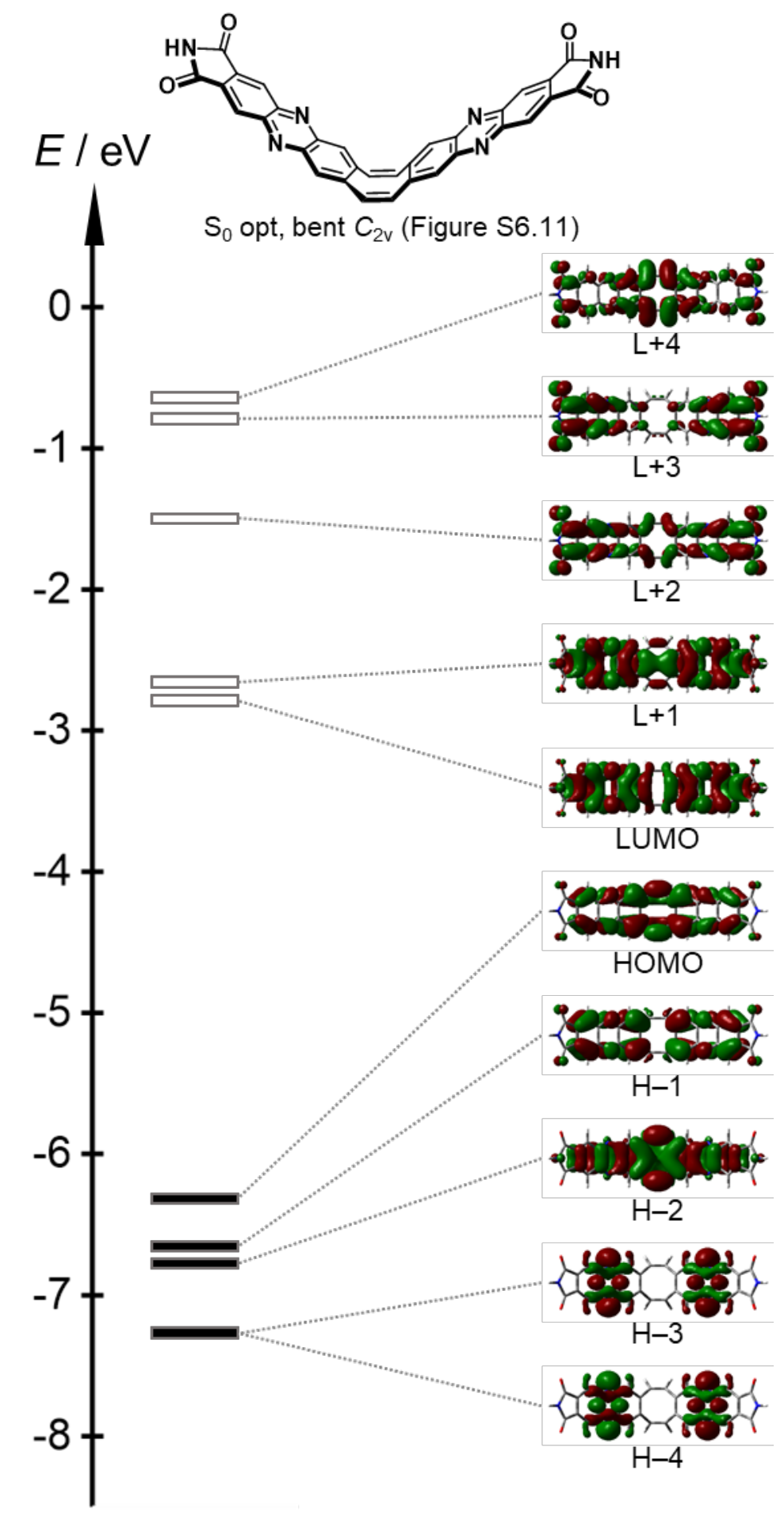

Figure S6.18. Kohn-Sham molecular orbitals and their energy levels of FLAP2' with different bent angles. The representative molecular orbitals and their energy levels were calculated at the TD PBE0/6-31+G(d) level.

Table S6.3. Excitation energy, configuration, and oscillator strength of the $S_{0} \rightarrow S_{n}(n \leq 5)$ transitions for the $S_{0}$ optimized FLAP2' at the TD PBE0/6-31+G(d) level.

\begin{tabular}{|c|c|c|c|}
\hline Transition & Excitation energy & Configuration & Oscillator strength \\
\hline $\mathrm{S}_{0} \rightarrow \mathrm{S}_{1}\left(A_{2}\right)$ & $2.85 \mathrm{eV}(435 \mathrm{~nm})$ & $\mathrm{H} \rightarrow \mathrm{L}(96 \%)$ & 0.0000 \\
\hline $\mathrm{S}_{0} \rightarrow \mathrm{S}_{2}\left(B_{1}\right)$ & $2.96 \mathrm{eV}(419 \mathrm{~nm})$ & $\mathrm{H} \rightarrow \mathrm{L}+1(94 \%)$ & 0.0204 \\
\hline $\mathrm{S}_{0} \rightarrow \mathrm{S}_{3}\left(B_{2}\right)$ & $3.04 \mathrm{eV}(408 \mathrm{~nm})$ & $\begin{array}{c}\mathrm{H}-4 \rightarrow \mathrm{L}+1(42 \%) \\
\mathrm{H}-3 \rightarrow \mathrm{L}(53 \%)\end{array}$ & 0.0215 \\
\hline $\mathrm{S}_{0} \rightarrow \mathrm{S}_{4}\left(A_{1}\right)$ & $3.04 \mathrm{eV}(407 \mathrm{~nm})$ & $\begin{array}{c}\mathrm{H}-4 \rightarrow \mathrm{L}(55 \%) \\
\mathrm{H}-3 \rightarrow \mathrm{L}+1(43 \%)\end{array}$ & 0.0002 \\
\hline $\mathrm{S}_{0} \rightarrow \mathrm{S}_{5}\left(B_{2}\right)$ & $3.19 \mathrm{eV}(389 \mathrm{~nm})$ & $\mathrm{H}-2 \rightarrow \mathrm{L}(83 \%)$ & 0.4128 \\
\hline
\end{tabular}




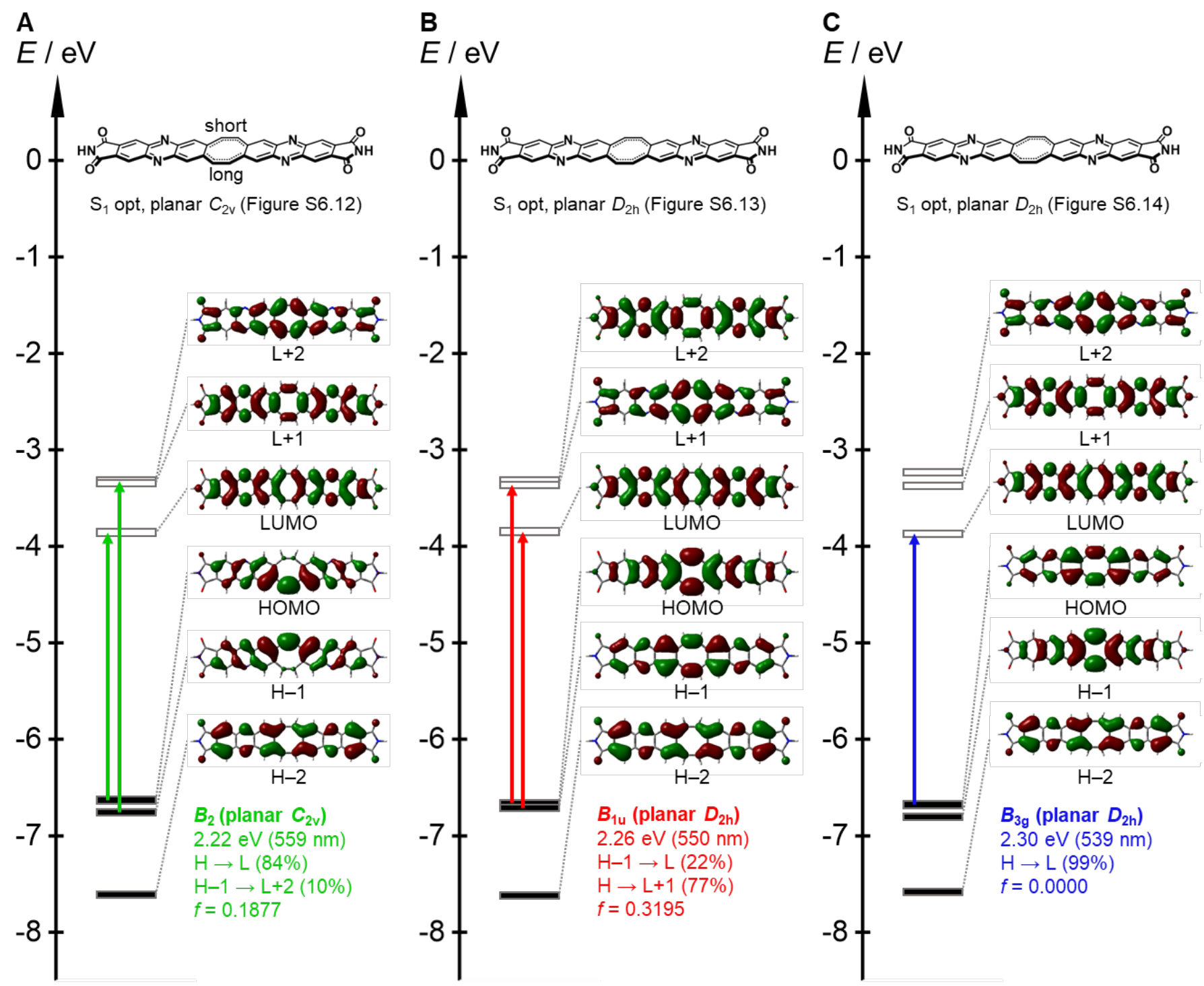

Figure S6.19. Kohn-Sham molecular orbitals and their energy levels of FLAP2' at the different $\mathrm{S}_{1}$ geometries in (A) Figure S6.12, (B) Figure S6.13, and (C) Figure S6.14, optimized at the TD PBE0/6-31+G(d) level of theory. 


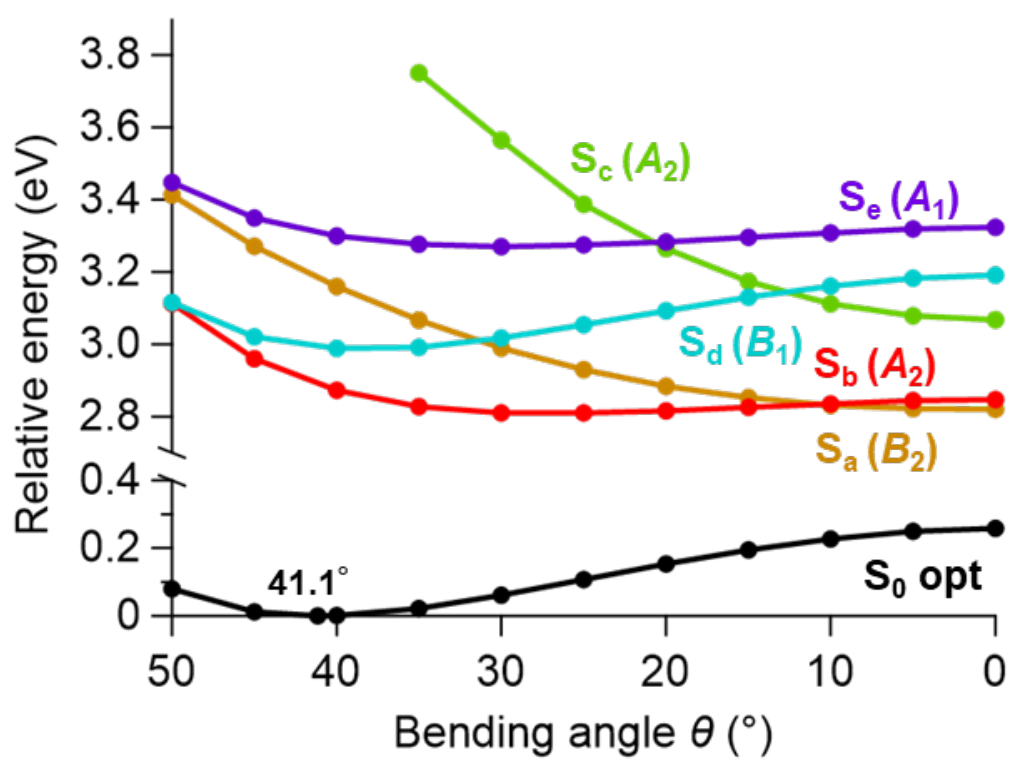

Figure S6.20. Potential energy diagram of the ground state $S_{0}$ and excited states $S_{a}, S_{b}, S_{c}, S_{d}$, and $S_{e}$ for FLAP0' with fixed bent angles of COT. The molecular geometries are optimized in $\mathrm{S}_{0}$.

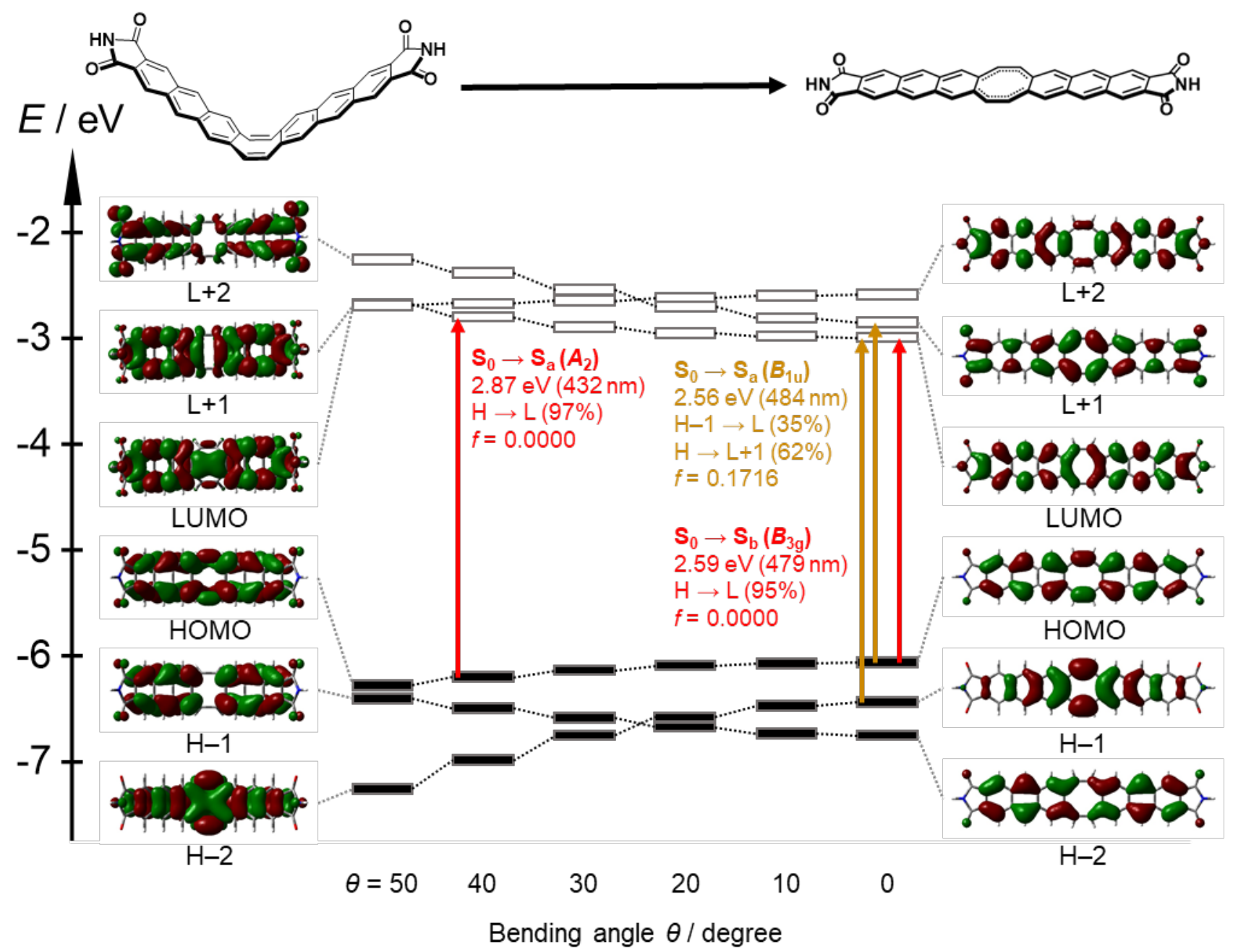

Figure S6.21. Kohn-Sham molecular orbitals and their energy levels of FLAP0' with different bent angles. DFT optimized geometries at the PBE0/6-31+G(d) level in $\mathrm{S}_{0}$ were used for the TD-DFT calculations at the same level. 


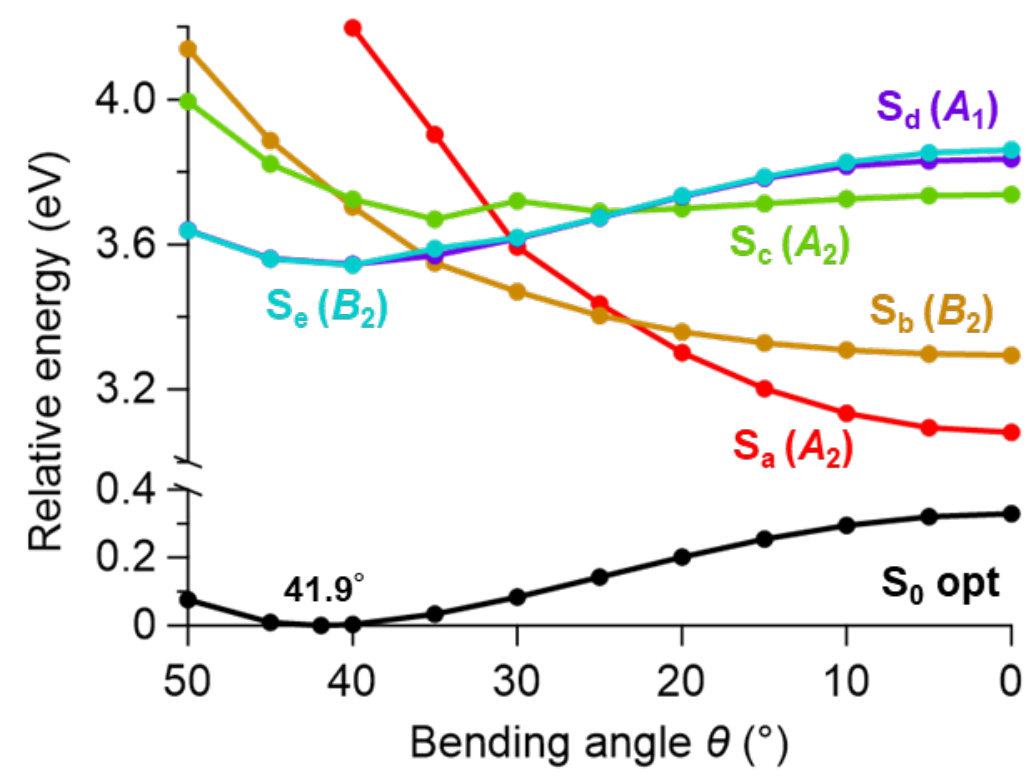

Figure S6.22. Potential energy diagram of the ground state $S_{0}$ and excited states $S_{a}, S_{b}, S_{c}, S_{d}$, and $S_{e}$ for FLAP1' with fixed bent angles of COT. The molecular geometries are optimized in $\mathrm{S}_{0}$.

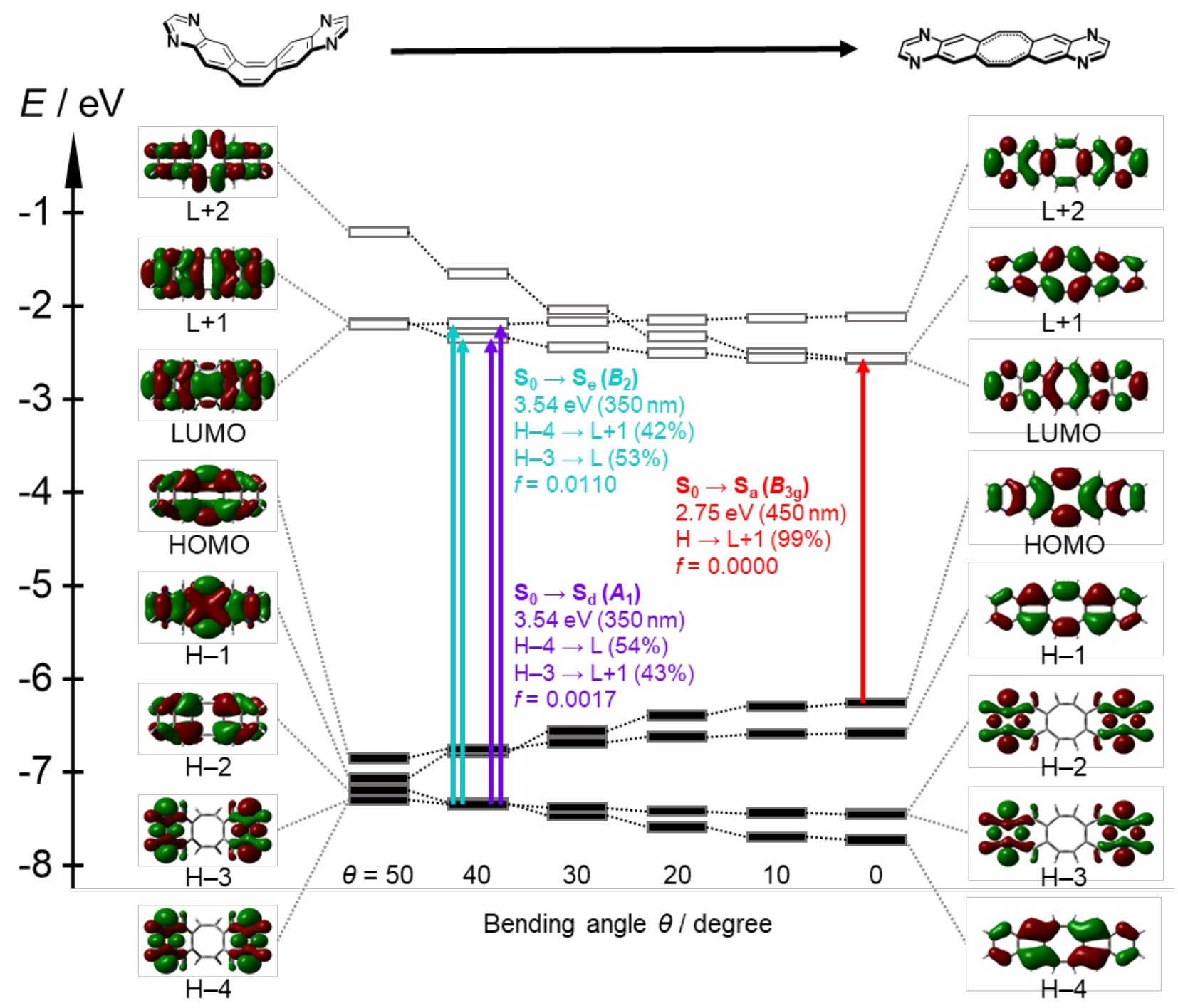

Figure S6.23. Kohn-Sham molecular orbitals and their energy levels of FLAP1' with different bent angles. DFT optimized geometries at the PBE0/6-31+G(d) level in $\mathrm{S}_{0}$ were used for the TD-DFT calculations at the same level. 


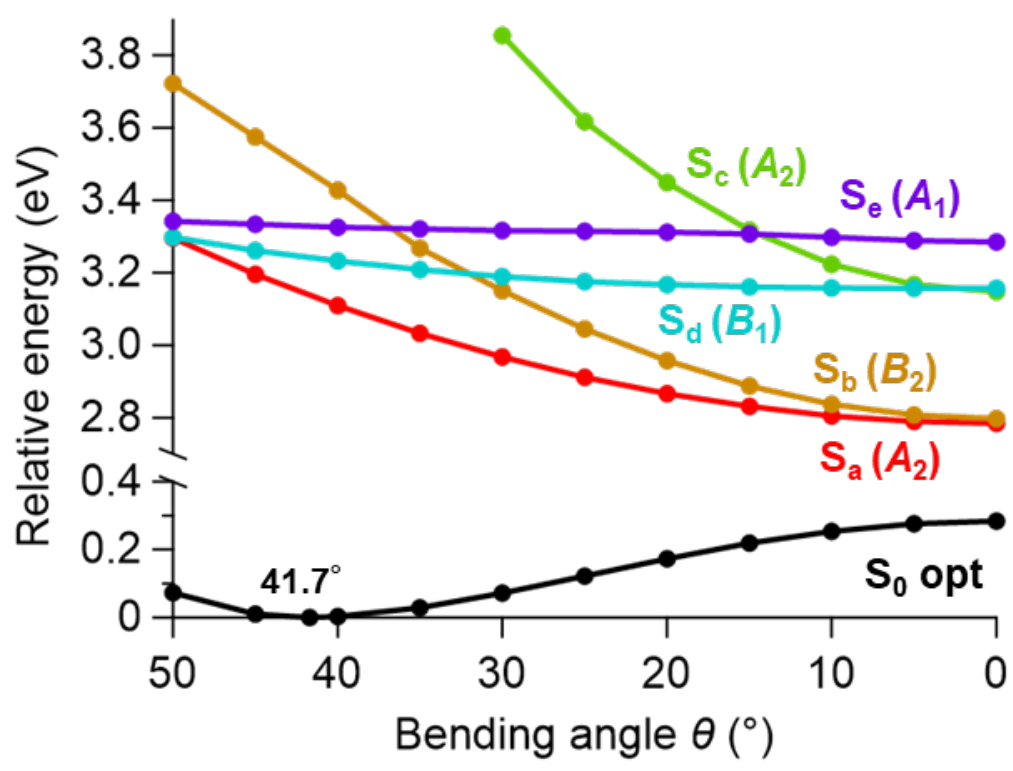

Figure S6.24. Potential energy diagram of the ground state $S_{0}$ and excited states $S_{a}, S_{b}, S_{c}, S_{d}$, and $S_{e}$ for FLAP2' with fixed bent angles of COT. The molecular geometries are optimized in $\mathrm{S}_{0}$.

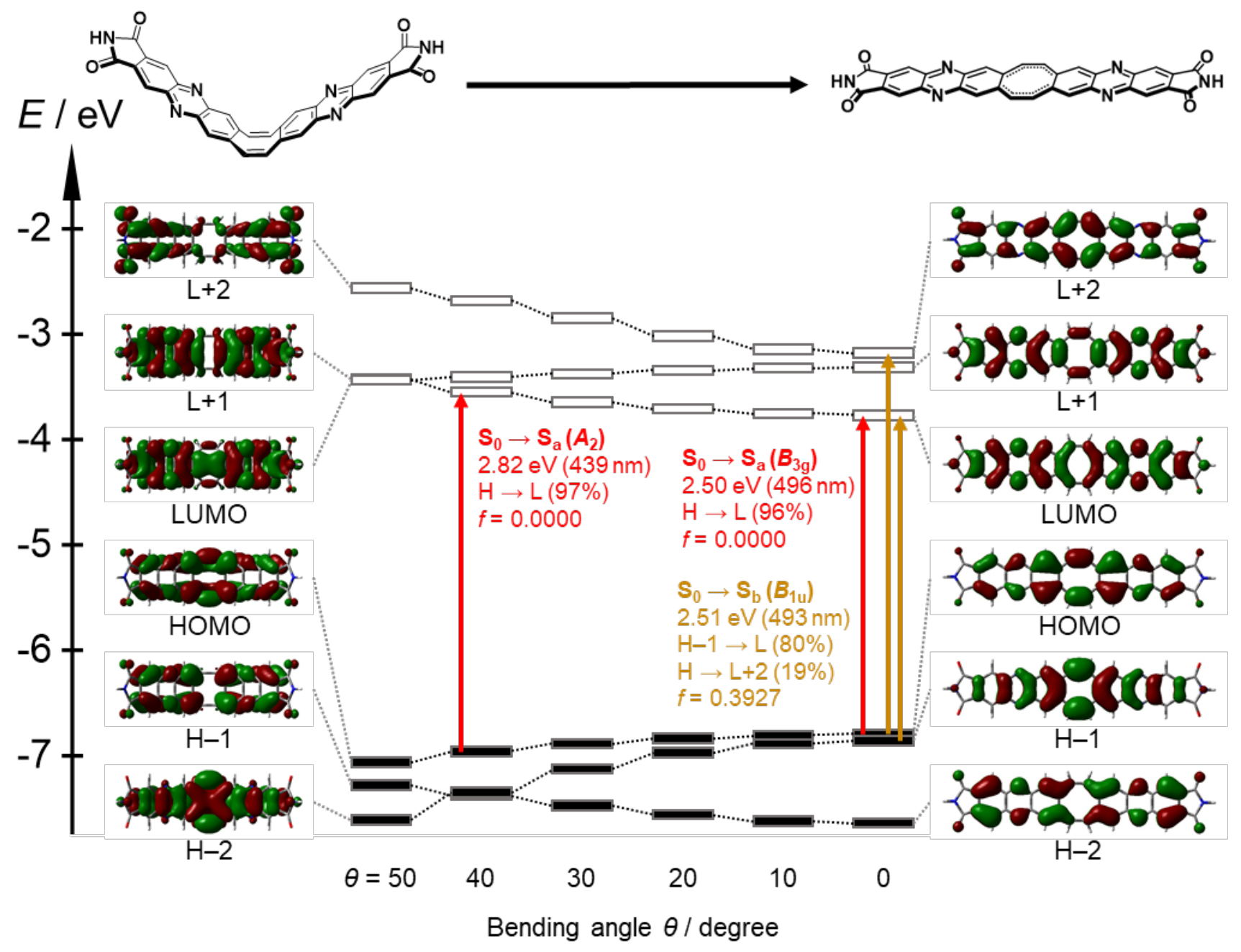

Figure S6.25. Kohn-Sham molecular orbitals and their energy levels of FLAP2' with different bent angles. DFT optimized geometries at the PBE0/6-31+G(d) level in $\mathrm{S}_{0}$ were used for the TD-DFT calculations at the same level. 


\section{Supporting references}

[S1] R. Kimura, H. Kuramochi, P. Liu, T. Yamakado, A. Osuka, T. Tahara, S. Saito, Angew. Chem. Int. Ed. 2020, 59, 16430-16435.

[S2] D. Kuzuhara, W. Furukawa, N. Aratani, H. Yamada, J. Porphyrins Phthalocyanines 2020, 24, 489-497.

[S3] R. Kotani, H. Sotome, H. Okajima, S. Yokoyama, Y. Nakaike, A. Kashiwagi, C. Mori, Y. Nakada, S. Yamaguchi, A. Osuka, A. Sakamoto, H. Miyasaka, S. Saito, J. Mater. Chem. C 2017, 5, 5248.

[S4] G. M. Sheldrick, Acta Crystallogr. 2015, A71, 3-8.

[S5] G. M. Sheldrick, Acta Crystallogr. 2015, C71, 3-8.

[S6] T. Förster, G. Hoffmann, Z. Phys. Chem. 1971, 75, 63.

[S7] R. Kotani, H. Sotome, H. Okajima, S. Yokoyama, Y. Nakaike, A. Kashiwagi, C. Mori, Y. Nakada, S. Yamaguchi, A. Osuka, A. Sakamoto, H. Miyasaka, S. Saito, J. Mater. Chem. C 2017, 5, 5248.

[S8] Gaussian 16, Revision A.03, M. J. Frisch, G. W. Trucks, H. B. Schlegel, G. E. Scuseria, M. A. Robb, J. R. Cheeseman, G. Scalmani, V. Barone, G. A. Petersson, H. Nakatsuji, X. Li, M. Caricato, A. V. Marenich, J. Bloino, B. G. Janesko, R. Gomperts, B. Mennucci, H. P. Hratchian, J. V. Ortiz, A. F. Izmaylov, J. L. Sonnenberg, D. Williams-Young, F. Ding, F. Lipparini, F. Egidi, J. Goings, B. Peng, A. Petrone, T. Henderson, D. Ranasinghe, V. G. Zakrzewski, J. Gao, N. Rega, G. Zheng, W. Liang, M. Hada, M. Ehara, K. Toyota, R. Fukuda, J. Hasegawa, M. Ishida, T. Nakajima, Y. Honda, O. Kitao, H. Nakai, T. Vreven, K. Throssell, J. A. Montgomery, Jr., J. E. Peralta, F. Ogliaro, M. J. Bearpark, J. J. Heyd, E. N. Brothers, K. N. Kudin, V. N. Staroverov, T. A. Keith, R. Kobayashi, J. Normand, K. Raghavachari, A. P. Rendell, J. C. Burant, S. S. Iyengar, J. Tomasi, M. Cossi, J. M. Millam, M. Klene, C. Adamo, R. Cammi, J. W. Ochterski, R. L. Martin, K. Morokuma, O. Farkas, J. B. Foresman, and D. J. Fox, Gaussian, Inc., Wallingford CT, 2016. 